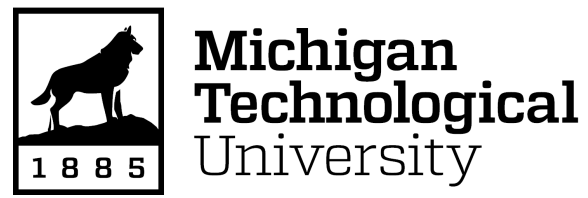

Michigan Technological University Digital Commons @ Michigan Tech

\title{
ACTIVATION SCAVENGING OF AEROSOL : EFFECT OF TURBULENCE AND AEROSOL-COMPOSITION
}

Abu Sayeed Md Shawon

Michigan Technological University, ashawon@mtu.edu

Copyright 2021 Abu Sayeed Md Shawon

\section{Recommended Citation}

Shawon, Abu Sayeed Md, "ACTIVATION SCAVENGING OF AEROSOL : EFFECT OF TURBULENCE AND AEROSOL-COMPOSITION", Open Access Dissertation, Michigan Technological University, 2021.

https://doi.org/10.37099/mtu.dc.etdr/1316

Follow this and additional works at: https://digitalcommons.mtu.edu/etdr

Part of the Atmospheric Sciences Commons, Climate Commons, and the Meteorology Commons 


\title{
ACTIVATION SCAVENGING OF AEROSOL : EFFECT OF TURBULENCE AND AEROSOL-COMPOSITION
}

\author{
By
}

Abu Sayeed Md Shawon

\begin{abstract}
A DISSERTATION
Submitted in partial fulfillment of the requirements for the degree of DOCTOR OF PHILOSOPHY

In Applied Physics

MICHIGAN TECHNOLOGICAL UNIVERSITY

2021
\end{abstract}

(C) 2021 Abu Sayeed Md Shawon 

This dissertation has been approved in partial fulfillment of the requirements for the Degree of DOCTOR OF PHILOSOPHY in Applied Physics.

Department of Physics

Dissertation Advisor: Dr. Will Cantrell

Committee Member: Dr. Raymond Shaw

Committee Member: Dr. Claudio Mazzoleni

Committee Member: Dr. Mike Larsen

Department Chair: Dr. Ravindra Pandey 

This dissertation is dedicated to my-

\section{AMMU \& ABBU}

Rashida Akter \& Late Mohd. Ismail Hossine

A person who never gave up on me, \&

A person whom I wish were here, for this day. 



\section{Contents}

List of Figures $\ldots \ldots \ldots \ldots \ldots \ldots \ldots \ldots \ldots \ldots \ldots$

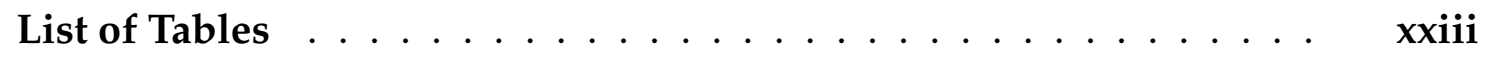

Acknowledgments ....................

Abstract $\ldots \ldots \ldots \ldots \ldots \ldots \ldots \ldots \ldots \ldots \ldots \ldots \ldots \ldots \ldots \ldots$

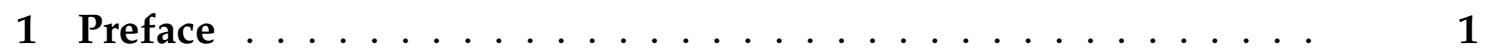

1.1 A Brief History . . . . . . . . . . . . . . . .

1.2 Motivation .................. 4

1.2.1 Aerosol-cloud-turbulence Interaction $\ldots \ldots \ldots$

1.2.2 Aerosol Scavenging . . . . . . . . . . . . . . 6

1.3 Outline .................... 8

2 Dependence of Aerosol-droplet Partitioning on Turbulence in a Laboratory Cloud . . . . . . . . . . . . . . . 11

2.1 Abstract..................... 13

2.2 Plain Language Summary _. . . . . . . . . . . . . 14

2.3 Introduction $\ldots \ldots \ldots \ldots \ldots \ldots \ldots \ldots$

2.4 Instrumentation . . . . . . . . . . . . . . . 19

2.5 Methods and Analysis . . . . . . . . . . 22

vii 
2.5 .1 Saturation Ratio . . . . . . . . . . . . . . 22

2.5.2 Cloud Droplets, Residuals, Interstitials, and Activated Fraction ................... 25

2.5.2.1 Cloud Droplets: . . . . . . . . . . . . . 25

2.5.2.2 Cloud Droplet Residuals: . . . . . . . . . $\quad 26$

2.5.2.3 Interstitial: . . . . . . . . . . . . . 26

2.5.2.4 Activated Fraction: . . . . . . . . . . . . 27

2.5.3 Turbulence in the Chamber . . . . . . . . . 28

2.6 Results and Discussion . . . . . . . . . . . . . . 29

2.6.1 Effect of Fluctuations on Aerosol Partitioning . . . . .

2.6.2 The Effect of Mean Forcing, $s_{0} \ldots \ldots \ldots \ldots$

2.6.3 Effect of Fluctuations in Forcing, $\sigma_{s_{0}} \ldots \ldots \ldots \ldots$

2.6.4 Effect of In-cloud Saturation Ratio, $S$ and $\sigma_{S} \ldots \ldots$

2.6.5 An Expression for Activated Fraction, $F_{n} \ldots \ldots \ldots$

2.7 Implications for the Atmosphere and Summary . . . . . . . 47

2.8 Acknowledgments $\ldots \ldots \ldots \ldots \ldots \ldots \ldots \ldots$

3 Dry Scavenging of Aerosol: Laboratory Investigation of the Effect of

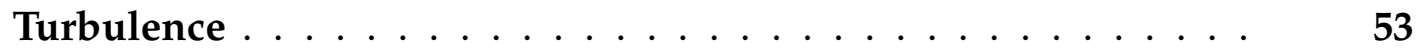

3.1 Introduction $\ldots \ldots \ldots \ldots \ldots \ldots \ldots \ldots \ldots \ldots$

3.2 Method and Instrumentation $\ldots \ldots \ldots \ldots$

3.3 Result and Discussion $\ldots \ldots \ldots \ldots \ldots$

3.3.1 Effect of Turbulence on $\tau_{\text {decay }} \ldots \ldots \ldots \ldots$

3.3.2 Other Preliminary Data . . . . . . . . . . . . 62

3.4 Summary ........................ 64 
4 To Improve the Temporal Resolution of a Dual Column CCN

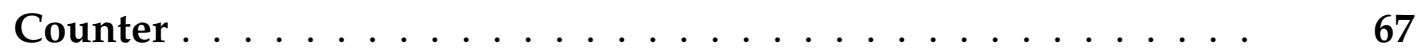

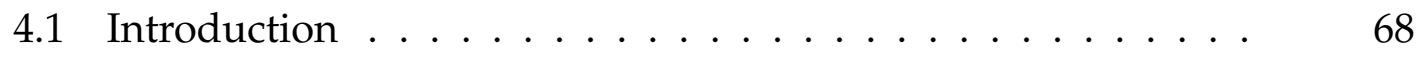

4.2 Method and Instrumentation . . . . . . . . . . 70

4.2.1 Parameter Setup ................ 70

4.2.2 Instrumentation ................. 72

4.2.3 Calibration method ............ 75

4.3 Result and Discussion ................ 79

4.4 Summary ............................... 81

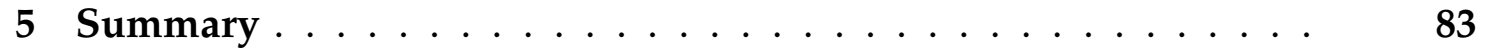

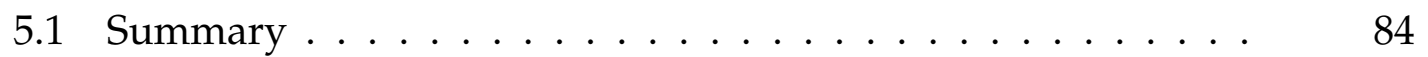

5.2 Future Possibilities ................... 85

A Letter of Permission ...................... 87

B Integral Diameter ..................... 91

C Supplementary Materials - Chapter $2 \ldots \ldots$

C.1 Size-selected Sampling of Aerosols . . . . . . . . . . . 96

D Supplementary Materials - Chapter $3 \ldots \ldots$

References ......................... 103 



\section{List of Figures}

1.1 A schematic showing the aerosol direct and aerosol indirect effect. Aerosol particles affect the radiative budget by directly scattering or absorbing solar radiation or by modulating cloud properties.

1.2 An illustration showing different sources and sinks of aerosol. The sources of aerosol can either be natural (e.g. mineral- or volcanicdust, sea-salt, etc.) or anthropogenic (e.g., ship track, industrial aerosol, etc.). Their sink mechanisms include dry- and different wet- depositions. In this dissertation, we focus on dry deposition and nucleation scavenging. $\ldots \ldots \ldots \ldots \ldots$

2.1 Schematic showing the instrumentation setup for the experiments. Atomizer generated aerosols are size selected using a DMA. The sampling inlet for the interstitial aerosol projected into the center of the chamber at the midplane. The interstitial outlet was $\sim 30 \mathrm{~cm}$ shorter than the inlet to avoid collecting the injected aerosols. The WELAS and PCVI were used to obtain the in situ size distribution of haze and cloud in the chamber and the size distribution of the cloud droplet residuals, respectively. . . . . . . . . . . . 
2.2 An illustration depicting how saturation ratio was modulated in the cloud chamber. The solid black curve represents the saturated vapor pressure of the flat surface of pure water (ClausiusClapeyron curve). The dotted straight lines represents the mixing line. Note that, both Clausius-Clapeyron curve and the mixing line are schematics (i.e., not scaled). A temperature difference between the top and bottom plates creates a supersaturation through the mixing process (subscripts $w$ and $s$ represent the water boundary and salt boundary respectively). An increase in $\Delta \mathrm{T}$ increases the mean supersaturation while salinity of the water at the bottom boundary decreases the equilibrium vapor pressure. A balance between the salt concentration at the bottom boundary and the changing $\Delta \mathrm{T}$ enables us to modulate the mean saturation ratio and its variance independently. . . . . . . . . . . . . .

2.3 Time series of the number concentration of interstitial aerosols, showing an approach to steady-state. The uncertainty bar (at approximately the 53rd minute) shows the first standard deviation around the mean value of $n_{i}$ at the steady-state. At the start of the aerosol injection, the source (injection) was greater than the sink (activation). Aerosol-cloud steady state was reached when source was balanced by the sink and the concentration of interstitial reaches a plateau. . . . . . . . . . . . . . . 
2.4 Size distributions of interstitial (solid, red) and residual (dashed, blue) aerosol for a turbulent, cloudy condition in the chamber. The shaded region indicates one standard deviation. $\mathrm{NaCl}$ was size-selected at $130 \mathrm{~nm}$ and injected at a constant rate of $\approx 170$ $\mathrm{cm}^{-3} \mathrm{~min}^{-1}$. The peak at $\approx 210 \mathrm{~nm}$ is from doubly charged particles, which have the same electrical mobility as singly charged $130 \mathrm{~nm}$ particles. The peak at $\approx 90 \mathrm{~nm}$ is from particles that were singly charged when they were injected into the chamber, but doubly charged when they were measured with the SMPS after being sampled from the chamber. The distributions shown here are the average of approximately 10 distributions collected over $30 \mathrm{~min}$ in steady state conditions. The size cut used for the PCVI was $4.5 \mu \mathrm{m}$ diameter. $\Delta T=18 \mathrm{~K}$ for these experiments. . . . . . . .

2.5 The size distribution of haze and cloud droplets measured from the chamber using the WELAS. Clean and polluted conditions are shown (blue circles and red squares respectively). The orange dotted line corresponds to the the cutoff diameter considered to delineate interstitials and droplets. The numbers in the parentheses indicate the corresponding aerosol injection rate. $\ldots \ldots \ldots$ 
2.6 Effect of $S_{0}$ on activation with fluctuations held constant, showing (a) activated fraction as a function of the mole fraction of water $\left(\chi_{\mathrm{H}_{2} \mathrm{O}}\right)$ at the bottom boundary, and (b) size distributions of interstitial aerosol and droplets for three different cases. $\chi_{\mathrm{H}_{2} \mathrm{O}}$ gives an estimation of the relative change in forcing, $S_{0}$, in the system. As $\chi_{\mathrm{H}_{2} \mathrm{O}}$ decreases $S_{0}$ also decreases. All these experiments had the same $\Delta T$ and the same injection rate of $\mathrm{NaCl}$ aerosol particles. The only variable was $S_{0}$. Thus, in panel (a), the largest activated fraction was achieved when we have only fresh water at the bottom, i.e., at the highest $S_{0}$. No activation was observed when the bottom boundary had the highest amount of salt, i.e., at lowest $S_{0}$. Size distributions shown in blue, red, and purple correspond to the three points marked I, II, and III in panel (a). The orange dashed line, showing the critical diameter of $130 \mathrm{~nm} \mathrm{NaCl}$ aerosol $\left(D_{c}\right)$, is the demarcation between the cloud and the haze droplets.

2.7 Probability density functions of $S_{0}$ for case A (left) and B (right). Note that the mean is constant, but that the width of the PDFs increase with $\Delta T$. There is an offset between the measured $S_{0}$ and actual values of $S_{0}$ in the system. However, the relative changes among different cases as well as the measured $\sigma_{S_{0}}$ are reliable [1]. 
2.8 Activated fraction as a function of $\Delta \mathrm{T}$ in the chamber. Blue (downward pointing) and red (upward pointing) triangles represent $F_{n}$ for cases A and B respectively. The mean saturation ratio was held constant for each case, but the fluctuations increased, as shown in Figure 2.7. The secondary $\mathrm{x}$ axis shows the turbulence energy dissipation rate corresponding to $\Delta \mathrm{T}$. Since case $\mathrm{A}$ had a lower initial saturation ratio, it also has a lower initial activated fraction.. .

2.9 Figure showing relationships between integral diameter and fluctuations in the chamber. Top: integral diameter as a function $\Delta T$ for both case A and B. Bottom left: Size distributions of particles in the chamber for case A. Bottom right: Size distributions of particles in the chamber for case B. Note that a constant $S_{0}$ was maintained for all the gradients in case $\mathrm{A}\left(S_{0,6}\right)$, and all the gradients in case B $\left(S_{0,9}\right)$. Only $\sigma_{S_{0}}$ was varied in these cases (see Figure 2.7). Note the differences in the number concentrations of particles in the chamber for the two cases. Though the injection rate is the same for all cases, the residence time of particles in the chamber changes, resulting in a different concentration of particles in the chamber. . . . . . . . . . . . . . 
2.10 Activated fraction, $F_{n}$, as a function of aerosol injection rate. Upward pointing triangles (blue) and downward pointing triangles (red) correspond to the smaller (singly charged, $130 \mathrm{~nm}$ ) and larger doubly charged particles (220nm, doubly charged) aerosol respectively. The lines are the linear fits calculated for each mode. The temperature difference in the chamber was held at $18 \mathrm{~K}$; thus the forcing, $S_{0}$, was constant in these experiments. Differences occur because the in-cloud saturation ratio, $S$, is changing. . . . .

2.11 Normalized size distribution of interstitial (solid lines) and cloud droplet residuals (dashed lines) from the cleanest (grey lines) and most polluted (red lines) cases shown in Figure 2.10. The distributions are normalized by the total number concentration of aerosols in each case. In the cleanest condition, there was almost no interstitial, with nearly all the aerosols found in residuals. However, in the polluted condition, the scenario was reversed, and more interstitials were found than residuals. $\ldots \ldots \ldots \ldots \ldots$

2.12 Schematic of the proposed model of $F_{n}$. The saturation ratio of the environment, $S_{e n v}$ is considered to have a Gaussian profile, with a mean $S$ (blue vertical line), and standard deviation, $\sigma_{S}$ (pink horizontal line). The red, dashed vertical line depicts the critical saturation of monodisperse and chemically homogeneous aerosol. The probability of a particle to be exposed to a saturation ratio larger than $S_{c}$ is represented by the shaded blue region. Eqn. 2.6 gives the area under the curve of that region. . . . . . . . . 
2.13 A schematic comparing the aerosol-limited (panel a) and updraftlimited (panel b) regimes described by [2], and the mean (panel a') and fluctuation dominated (panel $b^{\prime}$ ) regimes discussed here and by [3]. Note that the abscissas for panels $\mathrm{a}$ and $\mathrm{b}$ are the aerosol critical saturation ratio, $S_{c}$, while in $\mathrm{a}^{\prime}$ and $\mathrm{b}^{\prime}$, the abscissas are the saturation ratio in the environment, $S_{\text {env }}$. The variable considered changes because [2] considered polydisperse particles exposed to a single peak supersaturation in a parcel (vertical lines in the figure) while in [3] and here we have examined particles of a single size and composition (vertical lines in the figure) which are exposed to a distribution of saturation ratios in the environment.

3.1 An example of calculating $\tau_{\text {decay }}$ from linear fit from natural log of the measured number concentration. Only the $70 \mathrm{~nm}$ size bin was considered in this particular case. $\tau_{\text {decay,70 }}$ calculated from the fit was 90 minutes. The corresponding number concentration data and the exponential fit of the data are shown in the inset. The same method was applied for calculating $\tau_{\text {decay }}$ in other experiments as

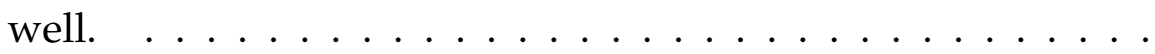

3.2 $\tau_{\text {decay }}$ as a function of $D_{p}$ at different $\Delta T$. Blue, red and black points correspond to $4 \mathrm{~K}, 12 \mathrm{~K}$, and $20 \mathrm{~K}$, respectively. $\tau_{\text {decay }}$ were calculated considering five neighboring bins of the selected sizes. The markers show the mean $\tau_{\text {decay }}$ of those five bins, and the uncertainty bars correspond to 1 standard deviation. . . . . . . .

3.3 (a) $\delta_{d b l}$ as a function of the mobility diameter, $D_{p}$ at different $\Delta T$. (b) $\delta_{d b l}$ as a function of $\Delta T$ for different sizes of the particles. . 
3.4 Figure showing the future experiments that can be performed to determine the effects of turbulence and quantify a residence time of aerosol as a function of size and turbulent intensity . . . .

4.1 Basic Illustration of a column of a DMT-CCNC. For a dual column DMT-CCNC, there are two similar columns. Aerosol flow, sheath flow, $T_{1}, T_{2}, T_{3}$, and OPC temperatures of each column can be controlled separately. The inner cylinder is made of thermally conductive materials and kept wet. A thermal difference, $\Delta T$ along $T_{1}$ and $T_{3}$ creates a supersaturation along the streamline of the aerosol flow. The inner cylinder illustrates the aerosol streamline. . . . . . . . . . . . . . . . . .

4.2 Schematic of the instrumentation setup. Size selected Ammonium Sulfate was used for the calibration. $M_{m}$ was used for maintain a constant flow through CPC. $M_{a}$, and $M_{b}$ controlled the flow through column A and column B respectively. . . . . . . . .

4.3 Example shows one of the flow cycles following eqn 4.1. Note the rising and lowering part of the flow denoted as up scan and down scan respectively. Blue and red curve shows the corresponding $Q_{T}$ of column A and column B. . . . . . . . . . . . . 
4.4 A schematic showing the work flow of the calibration process. Size selected ammonium sulfate particles were passed through a dual column DMT-CCN (with variable flow rate) and CPC (with constant flow rate). Then the time lag between their measurement has been calculated. Afterwards a set of rules were established to analyze the data.Up-scan and down-scan were considered separately for analysis. Ultimately we calculated the supersaturation as a function of $Q_{T} \ldots \ldots \ldots \ldots$

4.5 Example of a time Series of (a) Total flow $\left(Q_{T}\right)$ through column A and column B, (b) number Concentration of droplets $\left(N_{C C N}\right)$, (c) total number concentration of aerosol, $\left(N_{c p c}\right),(\mathrm{d})$ activated fraction, and (e) time lag in the measurement between DMT-CNN and CPC. In this case, column A and column B had $\Delta T=18 \mathrm{~K}$, and $\Delta T=6 \mathrm{~K}$ respectively. $140 \mathrm{~nm}$ diameter size-selected ammonium sulfate was used as aerosol. . . . . . . . . . . . .

4.6 Figure showing a calibration curve for column A and column B for the upscan, delineating a relation between total flow and the resulted supersaturation. This calibration curves were obtained averaging over 3 cycles for each diameter. Column A and and Column B was set at constant thermal gradient of $18 \mathrm{~K}$ and $6 \mathrm{~K}$. The green shadow shows the overlapping supersaturation at two different $\Delta T$ and different $Q_{T} \ldots \ldots \ldots \ldots$

4.7 Calibration curve for column A and column B for the downscan. All the other parameters are same as the up scan case (see figure

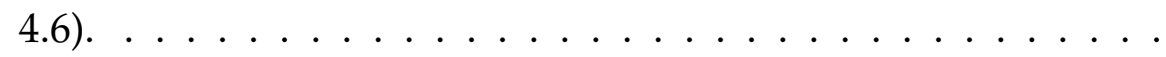


4.8 Time series of CCN counter measurement at the SGP. Quality control (QC) algorithm was applied to get a stable data set. Five supersaturation is achieved in one hour time $\ldots \ldots \ldots \ldots$

B.1 $I=n_{d} \bar{D}_{w}$ as a function of the mole fraction of water, $\chi_{\mathrm{H}_{2} \mathrm{O}}$, at the bottom boundary of the chamber. High $\chi_{\mathrm{H}_{2} \mathrm{O}}$ corresponds to the high $S_{0}$ and vice versa. $n_{d} \bar{D}_{w}$ increases monotonically with decreasing $S_{0}$ until $\chi_{\mathrm{H}_{2} \mathrm{O}}=0.93$. The decrease in $I$ after that point indicates that the mean saturation ratio was either subcritical or subsaturated and activation was dominated by the fluctuations. In such cases, an increase in the integral diameter is no longer proportional to the mean saturation ratio. . . . . . . . . .

C.1 Size selected $\mathrm{NaCl}$ was injected at a constant rate inside the chamber to create and sustain cloud condition. The first peak (A) and surrounding bins were the desired size bins. The secondary peak (B) and the surrounding bins were almost twice as big as the desired size bins which were selected by the DMA because of their same electric mobility. The small peak (C) on the left of the primary peak was our desired size with two charges. Note that, this figure shows the number concentration out of the atomizer. Inside the chamber this concentration has a dilution factor because of the volume difference between the cloud chamber and the atomizer. 
C.2 Time series of saturation ratio. The saturation ratio (yellow lines) was measured using the collocated LICOR (measuring vapor pressure) and RTD (measuring temperature). Both RTD and LICOR have the sampling frequency frequency of $1 \mathrm{~Hz}$. The blue line shows 5 min moving average. Please note the drop saturation ratio approximately around 2500th second. We started injecting aerosol around that time period. The profile of saturation ratio before aerosol injection corresponds to $S_{0}, \sigma_{S_{0}}$. The saturation ratio reaches a new steady state after brief transient which is the in-cloud saturation ratio $\left(S, \sigma_{S}\right) \ldots \ldots \ldots \ldots$

D.1 Figure showing the workflow to calibrate the instrument in the regular way. Ammonium sulfate was used as the test aerosol with known hygroscopicity. Six $\Delta T$ was used for getting the calibration curve in the end. . . . . . . . . . . . . . . . . 100

D.2 Sigmoid fit for the the plot Activated Fraction vs diameter at $\Delta T$ $=4 \mathrm{~K}$. The diameters that was chosen is shown in D.1. Critical dry diameter shown in the figure was obtained by determining $50 \%$ of the maximum activated fraction. The critical supersaturation of this critical dry diameter is the supersaturation obtained by the DMT-CCNC at 4 K thermal gradient. . . . . . . . . . . . 101

D.3 Calibration curve showing $\Delta \mathrm{T}$ vs supersaturation. The reason behind using $\Delta \mathrm{T}$ vs supersaturation (not other way around) is that the operating software requires the slope and y intersect in this particular way. . . . . . . . . . . . . . . . . . . 101 



\section{List of Tables}

3.1 A table showing some preliminary data from the experiments conducted to understand the effect of size on dry removal of aerosol in a turbulent environment. The column Instrument Used correspond to the instrument that was used to size-select the aerosol and measuring the size distribution afterwards. AAC $=$ Aerodynamic Aerosol Sizer, DMA = Differential Mobility Analyzer, CPC $=$ Condensation Particle Counter, and SMPS $=$ Scanning Mobility Particle Sizer. . . . . . . . . . . . . . . . . . . .

B.1 Integral diameter and activated fraction for constant $S_{0}$.(cases I and II $\ldots \ldots \ldots \ldots \ldots \ldots \ldots \ldots \ldots \ldots \ldots \ldots \ldots \ldots \ldots \ldots$ 



\section{Acknowledgments}

"Whoever enters the Way without a guide will take a hundred years to travel a two-day journey"

- Rumi, Masnavi

This dissertation is one of the outcomes of a half-an-era journey. I am deeply indebted to Dr. Will Cantrell for willing to be my guide for most of it. He introduced me to the world of aerosol, cloud, and atmospheric science. He has been excellent support for me during my years at Michigan Tech, particularly during my hard times. All the meetings with him were full of productive discussions. His suggestions always helped me in my research and professional decisions. He is my academic advisor, philosopher, and a perfect role model for my personal and professional life.

I want to express my profound appreciation to my committee members, Dr. Raymond Shaw, Dr. Claudio Mazzoleni, and Dr. Mike Larsen. Dr. Shaw was an integral part of all the research I have done in the cloud chamber. He was the source of invaluable insight regarding cloud microphysics, fluid mechanics, and turbulence. The ingenious suggestions and feedbacks from Dr. Larsen were very insightful and helped me think more diversely. The conversations and discussions with Dr. Mazzoleni were constructive, and they helped me along the way. Overall, this work would not have been without the exceptional support of this great committee.

I am incredibly grateful to Greg Kinney and Dr. Prasanth Prabhakaran. I learned my way around the chamber from Greg, and most of these experiments would 
not have been possible without his help. Prashanth trained me on turbulence through his excellent class and helped me understand the intricacies of wellguided experiments.

This dissertation includes some work at PNNL with the unwavering support of Dr. Swarup China. I very much appreciate the opportunity to work with him and Dr. Gourihar Kulkarni as an intern there. I also gratefully acknowledge the assistance of Ian Helman for his excellent help, especially with the dry removal experiments.

My special gratitude goes to Dr. Ravindra Pandey, our department chair. He gave me unparalleled support and valuable advice throughout my Ph.D. career. I would also like to thank Claire Wiitanen, Wil Slough, and all the other faculty and staff from the department of Physics for facilitating my research in one way or another.

Every step of this journey was made possible by my colleagues and friends. Special thanks to Jesse, Susanne, Sarita, Lata, Prasatnth, Kamal, Subin, and Andrew for the coffee breaks, discussions, and weekends at KBC. I am also grateful to all my friends from Bangladesh and Nepal, who made it feel like a family for me and helped me survive the snow and blizzards.

I would like to extend my sincere thanks to my professors from Bangladesh, Prof. Shamima K Choudhury, Prof. Golam Dastergir Al-Quaderi, and Dr. Mahabub Alam Bhuiyan without whom I would not be here working on my graduation. Special thanks to my friends in Bangladesh, Amrita, Ela, Rafee, Niloy, Rossi, Nico Bhai, Shahriar, Titas Apu, and many others. 
I also want to acknowledge the Department of Energy, National Science Foundation, and the Department of Physics at Michigan Tech for their financial support.

Lastly, however, most importantly, I am sincerely grateful to my family members, including Ammu, Bhaia, Bhabi, Tama, Auntou, Sajib, and Bini. From my very first semester, Bini was always there for me, even before she became my better half. This work would not have been possible, and I would not have survived the winters without her support throughout. 



\section{Abstract}

The interaction of aerosol particles with solar radiation is a significant contribution to the global radiation balance. The magnitude of this aerosol-radiation interaction, among other parameters, depends on different aerosol properties, including how readily these particles would act as cloud condensation nuclei $(\mathrm{CCN})$. These properties are governed by the formation and scavenging processes of aerosol. This dissertation explores some of these scavenging processes.

Favorable humidity and preexisting aerosol particles acting as $\mathrm{CCN}$ are the sine qua non conditions to form cloud droplets in Earth's atmosphere. Forming cloud droplets (known as activation), meanwhile, acts as a wet scavenging mechanism for those CCN. Given the required humidity, size, and chemical composition of an aerosol particle, determine its probability to activate. Through targeted experiments in a cloudy, turbulent environment in Michigan Tech's $\Pi$ chamber, we show that turbulent fluctuation blurs correspondence between activation and a particle's size and chemical composition. We also show that turbulence enhances the activation efficiency and can mimic the effect of heterogeneity in the size and chemical composition of the aerosol particles. In the absence of clouds, we discuss how turbulence affects the dry scavenging of aerosol particles. Finally, we propose an operational protocol to improve the temporal resolution of an instrument that counts the number of $\mathrm{CCN}$ present in an environment as a function of supersaturation (i.e., relative humidity $>100 \%$ ) 

"We have in this fine dust a most beautiful illustration of how the little things in this world work great effects in virtue of their numbers. The importance of the office, and the magnitude of the effects wrought by these less than microscopic dust particles, strike one with as great wonder, as the great depths and vast areas of rock which, the palaeontologist tells us, is composed of the remains of microscopic animals."

- Dr. John Aitken (1880)

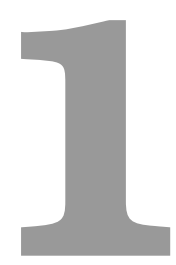

Preface 


\subsection{A Brief History}

Who among us has not been mesmerized by a giant puff billowing towards the endless sky and has never imagined a wild shape coming out of that? The Cloud Appreciation Society, in their manifesto, "pledged to fight blue-sky thinking" and said, "...clouds are the expressions of the atmosphere's mood... and... Life would be dull if we had to look up at cloudless monotony day after day..."[4]. From ancient times, clouds, in their all shapes and forms, fascinated the scientists and the litterateurs alike. And, the scientists and litterrateurs alike, before the late nineteenth century, no one ever pondered how the monotonous, inconspicuous dusts could be the reason behind those puffy, joyful clouds.

The importance of $d u s t$ particles in the air for cloudy condensation was first identified by Paul-Jean Coulier in 1875. During his experiments, Coulier noticed that condensation of water vapor required a fresh supply of air. This is the first hypothesis that considered the requirement of "condensation nuclei". However, having difficulties explaining some of his later results where condensation occurred without the supply of apparent fresh air, Coulier concluded that this requirement of condensation nuclei could not be a generalized condition [5, 6].

During 1880, completely unaware of Coulier's work, John Aitken carried out almost similar experiments using filtered and unfiltered air and discovered that the filtered air did not produce any condensation. From these observations, he hypothesized that having free surfaces (e.g., dusts) is required for water to condense (i.e., change its condition). Aitken, in this work, strongly argued that there 
would be no cloudy condensation; therefore, no fogs, mists, or cloud droplets without the presence of particles in the air [7]. Afterward, repeating Coulier's experiments, he also showed that the ambiguity of Coulier's later experiments came from the existing particles in the tube [5].

Though Aitken's experiments distinctly showed the necessity of preexisting particles for cloud formation, they could not predict its dependency on the properties of those particles. About fifty years later, Köhler developed a theory describing the equilibrium hygroscopic growth of aerosol. Köhler's work led to the detailed understanding of how the supersaturation along with the size, chemical composition, and morphology of aerosol particles affect this growth [8]. Supersaturation refers to a condition when the vapor pressure in air, $e$ is larger than an equilibrium value, $e_{S}$ at the same temperature (i.e., $\frac{e}{e_{s}}>1$ ).

One of the most crucial results from Köhler's work was to understand droplet formation as a threshold phenomenon. An aerosol particle gets activated (i.e., becomes a droplet) when it encounters a supersaturation larger than a critical value. After activation, it will grow continuously as long as the ambient environment is saturated. Turbulence affects this ambient supersaturation by affecting the scalars like temperature and vapor pressure. The effect of turbulence on the aerosol-cloud interaction (ACI) (i.e., aerosol-cloud-turbulence) was first reported by Kaufman and Tanré [9]. Through a simple aerosol growth model, they showed that a slight fluctuation in the ambient supersaturation could lead to a significant increase in the number of activated particles. A year later, Zapadinsky et al. pointed out that fluctuations in saturation ratio (due to turbulence or 
otherwise) may enhance the probability of heterogeneous nucleation [10]. Afterward, Kulmala et al. argued the possibility of subsaturated clouds in a turbulent environment [11].

A notable amount of work can be found discussing how turbulence affects the growth of droplets and its size distribution, even before Kaufman and Tanré [9]. In comparison, the effort to understand the effect of turbulence on aerosol partition, aerosol growth or activation is inadequate. Recent efforts that considered aerosol-cloud-turbulence interaction include Abade et al. and Chandrakar et al. [12, 13]. The investigation in this dissertation is our effort to explore further the effect of turbulence on aerosol activation in a laboratory setting and understand its implication to the atmosphere.

\subsection{Motivation}

\subsubsection{Aerosol-cloud-turbulence Interaction}

In the earlier part of the last decade, Koren and Feingold compared the system involving aerosol, cloud, and precipitation with a predator-prey system. Cloud, being the seed for rain, acts as prey while the latter acts as the predator forming on the existing cloud droplets. Aerosol plays the role of a modulator that may change clouds' properties through mere changes in number concentration [14].

The very first step for an aerosol-cloud-precipitation system is the activation of aerosol particles. Aerosol activation refers to the mechanism involving aerosol particles acting as the embryo for cloud droplets in a favorable humid condition. 
Primarily, activation of an aerosol particle depends on three parameters, size, chemical composition, and ambient supersaturation. Ambient supersaturation is affected by the turbulent forcing, creating conditions that either favor or oppose the activation process. Therefore, analogous to the aerosol-cloud-precipitation system, in the aerosol-cloud-turbulence system, one may consider aerosol acting as prey, cloud droplets acting as predators, and the turbulence forcing as the modulator.

Being part of two important systems, the interaction between aerosol and cloud plays a significant role in physiochemical and biogeochemical processes in the Earth system. Since precipitation is the primary mechanism to transport water from the atmosphere to the Earth's surface, this interaction affects the hydrological cycle. However, the most notable impact of ACI on the atmosphere comes from its interaction with solar and thermal radiation. Based on the temporal and spatial distribution, atmospheric aerosol particles scatter or absorb solar or thermal radiation and affect meteorological conditions such as temperature and stability. For example, on a cloudless day, aerosol particles can cool or warm the surface, depending on their scattering or absorbing properties. These effects on Earth's radiative budget are known as the direct aerosol effects.

In addition, aerosols serve to form clouds by acting as cloud condensation nuclei $(\mathrm{CCN})$ or ice nuclei (IN). Aerosol also influences properties like the lifetime or thickness of an existing cloud. These effects are known as the aerosol indirect effects. The first indirect effect refers to the increment of the optical depth of an existing cloud with increasing cloud droplet number concentration (CDNC) and fixed liquid water content (LWC), known as the Twomey Effect, or the Cloud 


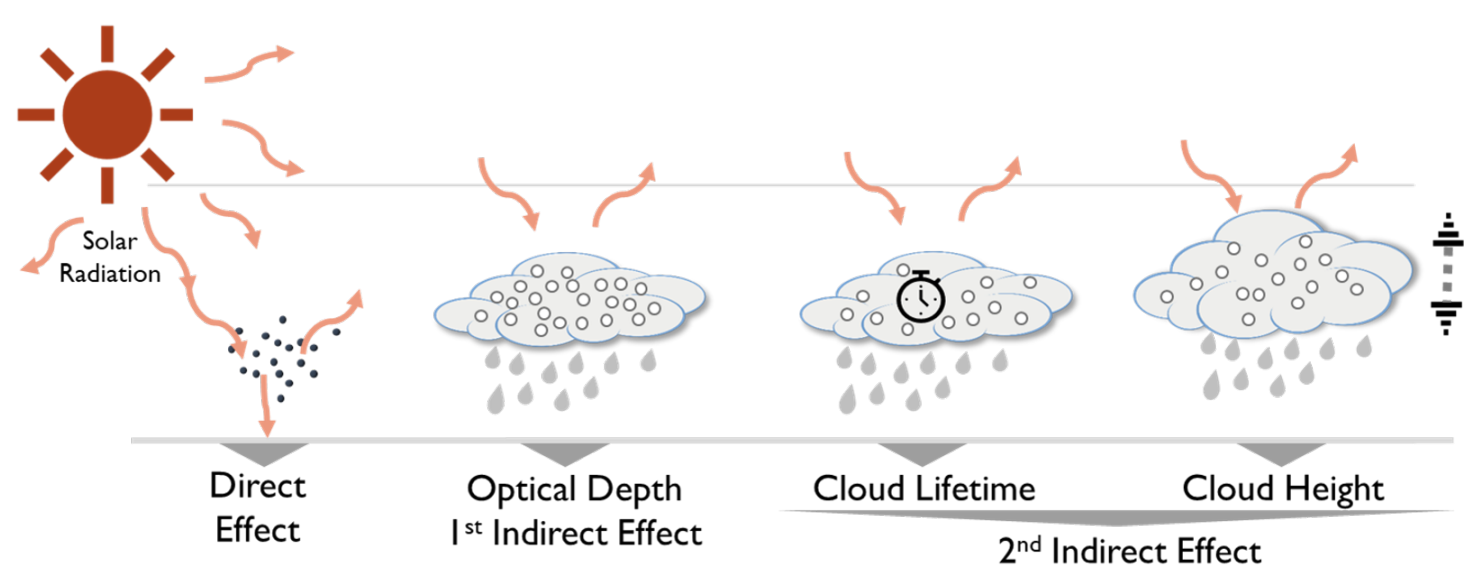

Figure 1.1: A schematic showing the aerosol direct and aerosol indirect effect. Aerosol particles affect the radiative budget by directly scattering or absorbing solar radiation or by modulating cloud properties.

Albedo Effect [15]. The second indirect effect includes the reduction of precipitation efficiency with the accretion of the smaller cloud droplets. Since this process increases the LWC, it eventually augments the cloud lifetime (Albrecht effect) [16], and the cloud thickness or cloud height [17, 18]. Although the mechanisms of these radiative effects due to $\mathrm{ACI}$ are well investigated in the literature, they cumulatively pose the largest uncertainty in the climate models.

\subsubsection{Aerosol Scavenging}

Exploring the properties of aerosol is the key element to understanding ACI. The life cycle and the behavior of atmospheric aerosols depend primarily on their interactions with various atmospheric processes and are characterized by their physical and chemical properties such as size distribution, mass, chemical composition, etc. These properties of aerosol vary depending on events like formation and removal. Understanding these mechanisms is essential to have a deeper appreciation of the role of aerosols in the atmosphere. Besides, since the 
aerosol particles determine many cloud properties, understanding the plausible removal mechanism is vital to simulate aerosol-climate effects.

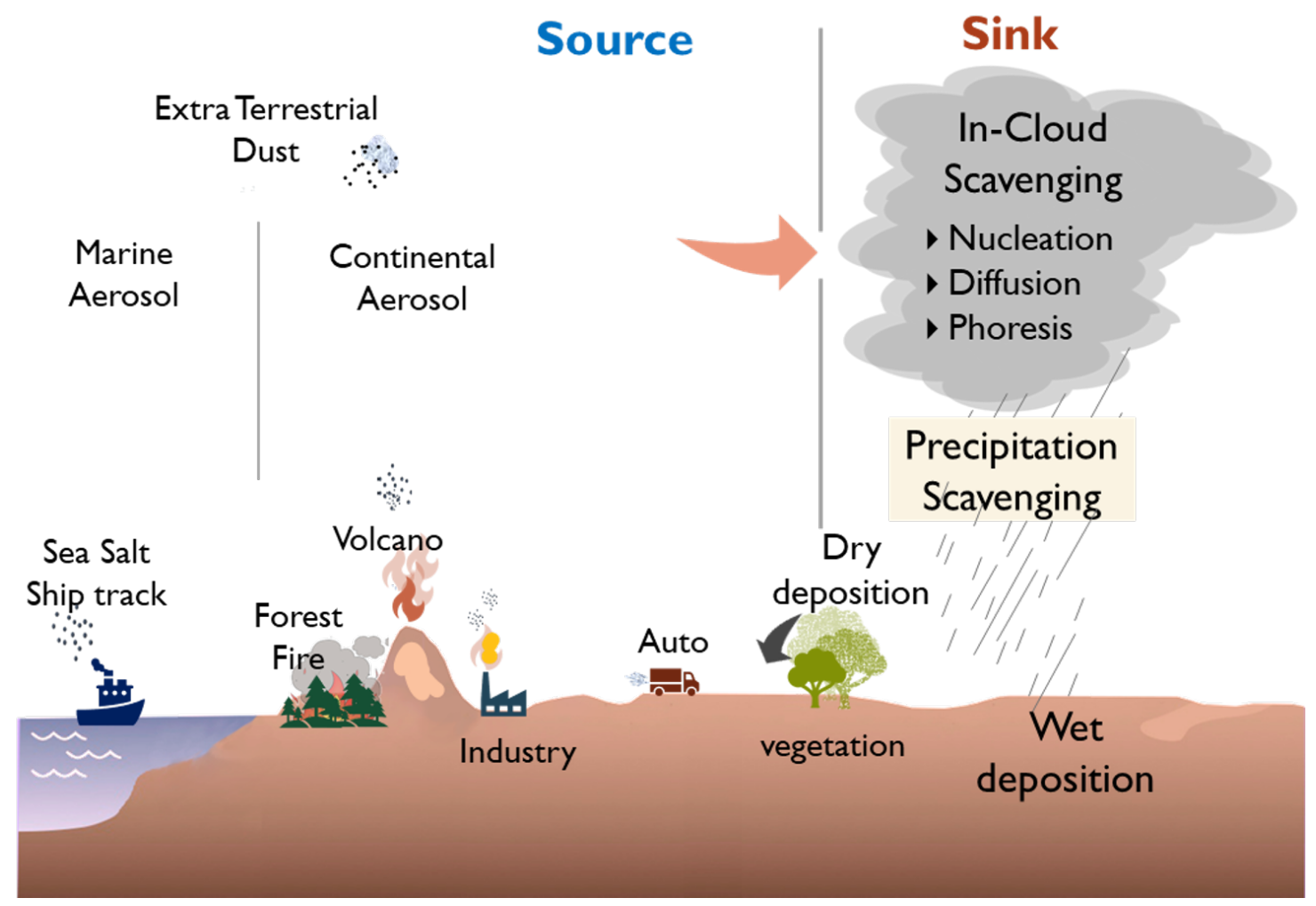

Figure 1.2: An illustration showing different sources and sinks of aerosol. The sources of aerosol can either be natural (e.g. mineral- or volcanic- dust, seasalt, etc.) or anthropogenic (e.g., ship track, industrial aerosol, etc.). Their sink mechanisms include dry- and different wet- depositions. In this dissertation, we focus on dry deposition and nucleation scavenging.

In general, the removal processes of aerosol are wet and dry deposition. Activation of preexisting aerosol in the atmosphere is one of the wet removal processes and the primary focus of this dissertation. Some aspects of the dry removal processes are also within the scope of this dissertation. 
The theories to understand the physics of turbulence effects on different collection processes are well developed and confirmed through simulation and experiments [19, 20]. Nonetheless, how turbulent flow enhances the collection efficiency has yet to be explored in detail. The motivation of this work is to improve our understanding of these aerosol scavenging processes in a controlled environment and in order to quantify the rate at which aerosol particles are removed by activation or dry deposition.

\subsection{Outline}

This dissertation is constituted of primarily three different chapters. Each of these self-contained chapters poses a different set of research questions.

Chapter 2, titled Dependence of Aerosol-droplet Partitioning on Turbulence in a Laboratory Cloud, discusses the effect of turbulence on the activation scavenging of aerosol. We explore the turbulence regime dependency on activation. The research questions we asked in this chapter are:

$\triangleright$ How does turbulence affect aerosol-droplet partitioning?

$\triangleright$ What is the relative contribution of -

- Mean Saturation Ratio, and

- Fluctuation in saturation ratio due to turbulence?

Chapter 3 explores the dry removal processes of aerosol in a turbulent environment. In this chapter, we discuss how turbulence influences the dry removal 
process. This investigation aims to quantify a rate of turbulence-induced dry removal and residence time of the aerosol particles in a Rayleigh-Bénard convection chamber. This chapter delineates some initial results and proposes a set of experiments to improve our understanding of the above-mentioned topics.

Chapter 4 focuses on instrumentation that measures the number concentration of cloud droplets as a function of supersaturation. We report a protocol to improve the temporal resolution of a dual-column cloud condensation nuclei counter.

The last chapter discusses the possible future work to further the study regarding aerosol scavenging through activation mechanism. 

Dependence of Aerosol-droplet Partitioning on Turbulence in a Laboratory Cloud 
This chapter is about the laboratory study of understanding the effect of turbulence on the aerosol activation process. It is based on a collaborative research published in JGR:Atmospheres $[\mathrm{A}] \mathrm{D}^{1}$ Supplementary materials of this chapter can be found in appendix A A brief discussion on the Integral Diameter can be found on appendix $B$ which is also related to this chapter.

\footnotetext{
${ }^{1}$ An edited version of this paper was published by AGU. Copyright (2021) American Geophysical Union
} 


\subsection{Abstract}

Activation is the first step in aerosol-cloud interactions, which have been identified as one of the principal uncertainties in Earth's climate system. Aerosol particles become cloud droplets, or activate, when the ambient saturation ratio exceeds a threshold, which depends on the particle's size and hygroscopicity. In the traditional formulation of the process, only average, uniform saturation ratios are considered. However, turbulent environments like clouds intrinsically have fluctuations around mean values in the scalar fields of temperature and water vapor concentration, which determine the saturation ratio. Through laboratory measurements, we show that these fluctuations are an important parameter that needs to be addressed to fully describe activation. Our results show, even for single-sized, chemically-homogeneous aerosols, that fluctuations blur the correspondence between activation and a particle's size and chemical composition, that turbulence can increase the fraction of aerosol particles which activate, and that the activated fraction decreases monotonically as the concentration of aerosol increases. Taken together, our data demonstrate that fluctuations can have effects equivalent to the aerosol limited and updraft limited regimes, known from adiabatic parcel theory. 


\subsection{Plain Language Summary}

Formation of cloud droplets occurs when the relative humidity exceeds a threshold value, inducing condensation of water vapor onto preexisting aerosol particles. Which of the preexisting particles become cloud droplets depends on their size and chemical composition. In the conventional view of the process, only the average value of the relative humidity is considered. Our laboratory experiments show that fluctuations, caused by turbulence, must also be considered. These fluctuations can increase the fraction of aerosol particles which become cloud droplets and, in general, can mimic the effect of heterogeneity in size and chemical composition of aerosol particles. 


\subsection{Introduction}

In the late nineteenth century John Aitken showed the importance of dust particles in the formation of fogs and clouds [5]. Since that beginning, the importance of aerosol-cloud interactions for climate as well as the hydrological cycle has been well established [21, 22, 23]. One of the best known examples of aerosolcloud interactions, the first indirect effect, is that, for constant liquid water content, an increase in the number of aerosol particles increases the concentration of droplets and eventually creates a cooling effect [15, 24]. A change in the aerosol particle loading can also change cloud lifetime [16] and thickness [17]. In a mixed phase cloud, the aerosol concentration may affect the number of ice crystals, increasing the precipitation efficiency [25]. Clearly, the concentration of aerosol particles in the atmosphere plays a crucial role in determining their interactions with clouds. In turn, aerosol number concentration is determined by the net difference of source and sink processes, i.e. how they are formed and how they get removed from the atmosphere.

The primary removal mechanism for accumulation mode particles (those with a diameter on the order of a tenth of a micrometer) is incorporation into a cloudprecipitation system [26]. Particles of this size range are neither small enough to be to be lost efficiently by diffusive process nor large enough to fall out by gravitational settling quickly. However, most accumulation mode particles readily act as cloud condensation nuclei (CCN), form cloud droplets, and can ultimately be removed from the atmosphere by precipitation. 
The aerosol particle-cloud droplet transition, known as activation and described by Köhler theory, occurs in the atmosphere when aerosol particles take up water in response to an increase in the environmental saturation ratio. When a particle reaches a critical diameter, condensation occurs spontaneously and it is considered activated. The saturation ratio $\left(S \equiv \frac{e}{e_{S}(T)}\right)$ or supersaturation $(s \equiv S-1)$ at which this occurs is known as the critical supersaturation, $s_{\mathcal{c}}$. (Here, $e$ is the partial pressure of water vapor and $e_{S}$ is the saturation vapor pressure of water at temperature T.) The critical diameter and supersaturation are functions of the size and chemical composition of the particles. Lower critical supersaturations (larger critical diameters) are characterized by larger dry sizes and more soluble substances.

As supersaturation increases, for example in a rising parcel of air undergoing adiabatic expansion, more and more aerosol particles activate. With an increasing number of cloud droplets, the sink for excess water vapor increases until, eventually, the condensation depletes supersaturation faster than expansion creates it. The maximum supersaturation reached then determines the number concentration of cloud droplets. (Droplet concentration can change due to other processes later in the cloud's life cycle.) This process is generally described in terms in which the values of $e$ and $T$ are uniform throughout the parcel. In other words, the supersaturation has a single, uniform value in the parcel, and the peak supersaturation is a single value [27, 28].

However, the assumption of a single peak saturation ratio is adequate only for a non-turbulent environment. In a turbulent environment, because of spatial 
and temporal variations in the values of scalar quantities like water vapor pressure and temperature, the saturation ratio within a parcel is characterized not by a single value but rather by a distribution of values. Recent field measurements suggest that this variability in supersaturation in a stratocumulus cloud can reach 0.1 to $0.3 \%$ [29]. The effect of variability in the saturation ratio has been considered in theory and models, showing that it can be significant. [9] argued that fluctuations in supersaturation in stratocumulus clouds could activate even small sulfate particles, which could then add mass through aqueous phase sulfate production. They proposed that such a mechanism could quadruple the effect of anthropogenic emissions of $\mathrm{SO}_{2}$ on climate through cloud processes. [11] pointed out that, even though activation strongly depends on the mean saturation ratio, a particle can grow in a stochastic saturation field with a subsaturated mean. More recently, a super-droplet method was implemented by [12] to address the difference in efficiency of aerosol activation in a mean-plus-fluctuation field. They suggested that, in a turbulent parcel, even if the mean supersaturatioin is subcritical, some "lucky" aerosols might get activated because of the fluctuations.

A wider range of literature may be found discussing the effect of turbulence on the cloud droplet distribution. [30] and [31] developed theoretical frameworks which suggested that both small- and large-scale turbulence broadens the droplet distribution. They argued that the close link between updraft velocity and supersaturation breaks in a turbulent environment. This phenomenon causes droplets to be exposed to different supersaturation values and eventually causes broadening in the droplet size distribution. [32] and [33] further explored 
the relevance of the phase relaxation time to this kind of broadening. Another mechanism for producing supersaturation fluctuations that may contribute to the broadening of droplet size distributions is the clustering of droplets in turbulence due to their finite inertia [34, 35]. In all of those scenarios, however, droplet growth is explored without consideration of the aerosol activation process. Activation is fundamentally different from droplet growth because it is a threshold phenomenon, implying that a single fluctuation can lead to a change in the system that persists for long times. How supersaturation fluctuations combine to affect both activation and droplet growth together is a more complex problem that is not considered here.

Laboratory investigations of the role of fluctuations in cloud microphysics have been more sparse, primarily because of the difficulty inherent in subjecting a parcel of air to a well characterized, turbulent, cloudy environment. The recent development of Michigan Tech's $\Pi$ chamber has enabled experiments where the role of fluctuations could be explored [33, 36, 37, 38, 39] though the primary focus to date has been on effects on the cloud droplet size distribution. However, [13] explicitly considered activation and scavenging, showing that in the $\Pi$ chamber's turbulent environment, aerosol particles of the same size and chemical composition could be found simultaneously in both cloud droplets and unactivated haze drops. More recently, [3] suggested three regimes of activation in a turbulent cloud, based on values of particles' critical saturation ratios relative to the environmental saturation ratio, using experiments from the $\Pi$ chamber as motivation. 
In this study, we use the $\Pi$ chamber to explore the effect of the mean saturation as well as the fluctuations that occur due to turbulence on aerosol activation. In previous experiments, the mean saturation ratio and fluctuations about the mean were inextricably linked. Here, we have developed a procedure to study the two independently. In the following two sections, we describe our experimental setup and analyses. Following that, results are presented and discussed. The last section includes a discussion of implications for the atmosphere and a brief summary of our study.

\subsection{Instrumentation}

Experiments were carried out in a multiphase, turbulent chamber, known as the $\Pi$ Chamber. It is a Rayleigh-Bénard convection chamber with a vertical separation of $1 \mathrm{~m}$ between the top and bottom plates. A cylindrical insert reduces the volume of the $4 \mathrm{~m}^{3}$ cuboid to $3.14 \mathrm{~m}^{3}$. The temperatures of the lower and upper control surfaces, as well as the side walls, can be controlled independently. Moist filter paper is generally used to keep all the walls saturated throughout the experiments. A supersaturated environment in the chamber is generally created through turbulent mixing. When an unstable temperature difference, $\Delta \mathrm{T}$, is set between the top and bottom plates, positively buoyant, moist air from the warmer bottom plate rises and mixes with negatively buoyant, moist air from the colder top plate, creating a supersaturated condition. The temperature of the

side walls are kept at the bulk temperature to minimize their effect on values of temperature and water vapor concentration [40]. More details of the $\Pi$ chamber are discussed in [36]. 
As noted above, turbulent mixing creates a supersaturated environment. To create a cloud, aerosol particles or cloud condensation nuclei $(\mathrm{CCN})$, are injected into the chamber. We used sodium chloride $(\mathrm{NaCl})$, generated using a constant output atomizer (TSI model 3076). The atomizer produces wet, polydisperse particles. After drying using a diffusion drier, the desired size distribution was selected using a differential mobility analyzer (DMA, TSI model 3071). We selected a mobility diameter of $130 \mathrm{~nm}$ unless otherwise noted in the text. The size selected aerosol were then injected into the center of chamber, at the midplane, with a flow rate of 2 slpm. A schematic of the cylindrical insert along with the aerosol injection and sampling systems is shown in Figure 2.1. Further details, including the dry size distribution of the aerosol injected, are shown in the Supporting Information.

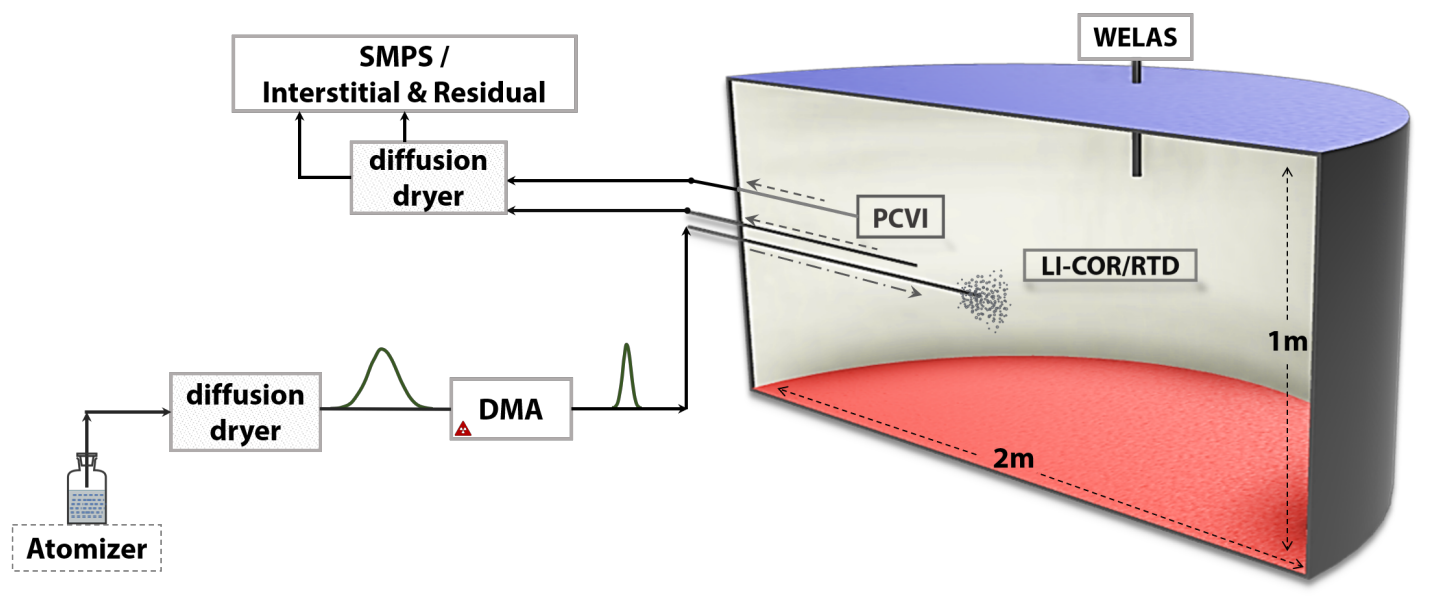

Figure 2.1: Schematic showing the instrumentation setup for the experiments. Atomizer generated aerosols are size selected using a DMA. The sampling inlet for the interstitial aerosol projected into the center of the chamber at the midplane. The interstitial outlet was $\sim 30 \mathrm{~cm}$ shorter than the inlet to avoid collecting the injected aerosols. The WELAS and PCVI were used to obtain the in situ size distribution of haze and cloud in the chamber and the size distribution of the cloud droplet residuals, respectively. 
We sampled aerosol particles from the chamber using a $6.35 \mathrm{~mm}$ od stainless steel tube projecting into the chamber at the midplane, $30 \mathrm{~cm}$ from the center. Particles with diameters $\leq 1 \mu \mathrm{m}$ diameter in the humid conditions in the chamber are collected, dried by diffusion drier, and sized using a Scanning Mobility Particle Sizer (SMPS, TSI model 3080); larger particles fall out of the sampling tube before exiting the chamber. This data is a measure of the dry size distribution of the interstitial particles. We measured the size of hydrated interstitial aerosol and cloud droplets directly with a WELAS optical particler counter (digital 2000, PALAS), in the size range 0.6 to $40 \mu \mathrm{m}$ diameter. In some experiments, cloud droplets are collected through a pumped counter flow virtual impactor (PCVI, Brechtel model 8100). The droplets were dried and the size distribution of the residuals measured with the SMPS. Note that we have only a single SMPS system; the size distributions of interstitials and residuals were not measured simultaneously. However, they were both measured in the same steady state conditions.

The saturation ratio in the chamber was calculated using collocated measurements of water vapor concentration and temperature near the center of the chamber. An open path $\mathrm{H}_{2} \mathrm{O}$ analyzer (LI-COR $7500 \mathrm{~A}$ ) was used to measure the water vapor concentration at $1 \mathrm{~Hz}$. The LI-COR has a working path length of $12.5 \mathrm{~cm}$ and averages over approximately $10 \mathrm{~cm}^{3}$. Four resistance temperature detectors (RTDs) were set up to measure the temperature across the working length of the LI-COR. The RTDs measured the temperature at the same rate as the LI-COR. The measurements of water vapor concentration and temperature 
were then used to calculate saturation ratio. While we cannot determine the absolute value of the saturation ratio obtained in this manner accurately enough to use for cloud microphysics calculations, previous work has indicated that the relative changes are reliable [1]. In other words, there is almost certainly an offset in the value of $S$ derived in this manner, but the $\sigma_{S}$ is sound.

\subsection{Methods and Analysis}

\subsubsection{Saturation Ratio}

As noted in the beginning of Section 2.4, the supersaturation in the $\Pi$ chamber is created through a turbulent mixing process, initiated by an unstable $\Delta \mathrm{T}$. The environment initially is cloud-free (because of the absence of $\mathrm{CCN}$ ) and does not contain any droplets. We denote the mean and variation of the saturation ratio for these conditions as $S_{0}$ and $\sigma_{S_{0}}\left(s_{0}\right.$ or $\sigma_{S_{0}}$ in terms of supersaturation). These are the initial conditions. Injecting aerosol (i.e. $\mathrm{CCN}$ ) at a constant rate into this supersaturated environment creates a cloudy condition and after a brief transient the system reaches a new, lower, steady-state supersaturation, $s$. This new supersaturation is the in-cloud supersaturation. The mean, $s$, and variation, $\sigma_{s}$, of in-cloud supersaturation can be modulated by changing the number concentration of injected aerosol. However, changing the aerosol number concentration changes both $s$ and $\sigma_{s}$ simultaneously.

The evolution of supersaturation in the cloud chamber can be described as the combination of source and sink term like in a natural cloud, and can be written 
as [3, 33, 41]-

$$
\frac{d s}{d t}=\frac{s_{0}-s}{\tau_{t}}-\frac{s}{\tau_{c}}
$$

where $\frac{s_{0}-s}{\tau_{t}}$ is the source term which is similar to the quantity $\alpha w$ used in the adiabatic parcel model; $\alpha$ is a thermodynamic factor and $w$ is the updraft velocity of the parcel. Here, $\tau_{t}$ is the turbulence correlation time and $\tau_{c}$ is the characteristic time scale that $s_{0}$ requires to reach the new, steady state value, $s$. This time scale is known as the phase relaxation time and defined as

$$
\tau_{c}=\left(2 \pi \Lambda n_{d} \bar{D}_{w}\right)^{-1}
$$

where $\Lambda$ is the diffusion coefficient of water vapor in air, modified to account for the release of latent heat as water condenses, $n_{d}$ is the number concentration of cloud droplets, and $\bar{D}_{w}$ is the mean cloud droplet diameter [42]. The product $n_{d} \bar{D}_{w}$ is defined as the integral diameter.

For an experiment in typical conditions, the temperature difference between the bottom and top boundaries, $T_{\text {bottom }}-T_{\text {top }} \equiv \Delta T$ induces an isobaric mixing which leads to a supersaturated condition. Since this mixing process is inherently turbulent, an increase in $\Delta T$ results in larger values of $s_{0}$ and an increase in the turbulent intensity. As a result, the fluctuations about the mean value, (i.e. $\left.\sigma_{s_{0}}\right)$ increase with $\Delta T$. A measurement of the turbulent intensity is discussed in section 2.5.3. Figure 2.2 is a schematic showing how mixing leads to a mean supersaturation. (See [43] for further discussion of supersaturation produced by isobaric mixing.) 


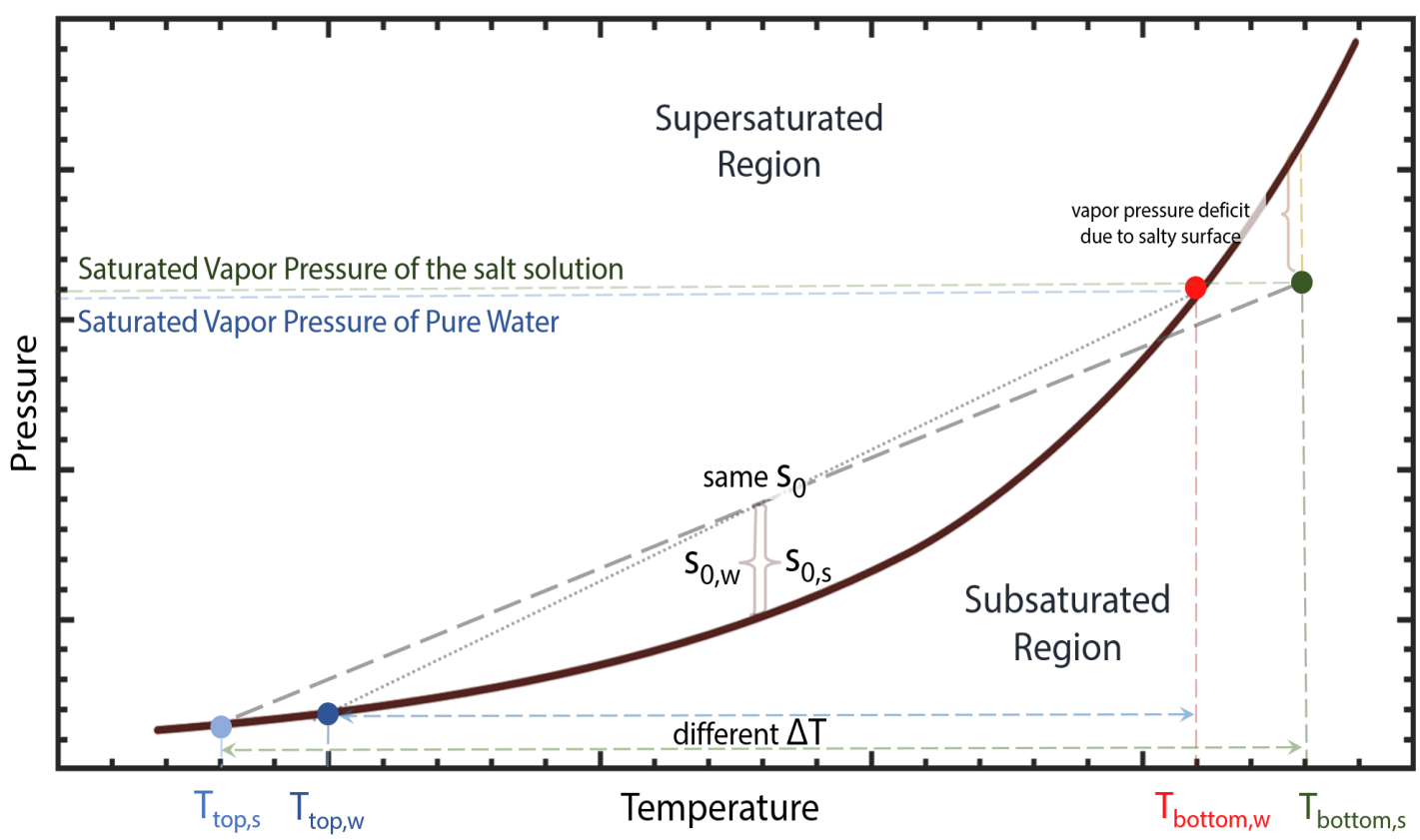

Figure 2.2: An illustration depicting how saturation ratio was modulated in the cloud chamber. The solid black curve represents the saturated vapor pressure of the flat surface of pure water (Clausius-Clapeyron curve). The dotted straight lines represents the mixing line. Note that, both Clausius-Clapeyron curve and the mixing line are schematics (i.e., not scaled). A temperature difference between the top and bottom plates creates a supersaturation through the mixing process (subscripts $w$ and $s$ represent the water boundary and salt boundary respectively). An increase in $\Delta \mathrm{T}$ increases the mean supersaturation while salinity of the water at the bottom boundary decreases the equilibrium vapor pressure. A balance between the salt concentration at the bottom boundary and the changing $\Delta \mathrm{T}$ enables us to modulate the mean saturation ratio and its variance independently.

In the typical configuration, it is not possible to decouple $s_{0}$ and $\sigma_{s_{0}}$. Both are controlled by the magnitude of $\Delta T$. To vary the two independently, another degree of freedom is needed. We achieve this by replacing the wet filter paper at the bottom boundary with a shallow pool of water $(\approx 1 \mathrm{~cm}$ depth), to which salt can be added (we use $\mathrm{NaCl}$.) At the same temperature, the equilibrium vapor pressure over a salt solution is lower than over pure water. Thus, by simultaneously decreasing the vapor pressure at the bottom boundary by adding salt to 
the pool and increasing the temperature difference between the plates, the mean supersaturation, $s_{0}$, can be matched, but at a different $\sigma_{S_{0}}$ because of the larger temperature gradient. This is shown schematically in Figure 2.2 .

\subsubsection{Cloud Droplets, Residuals, Interstitials, and Activated}

\section{Fraction}

\subsubsection{Cloud Droplets:}

In our analyses, the critical diameter, $D_{c}$, was calculated using a single parameter form of the Köhler equation based on the hygroscopicity $(\kappa)$ of the aerosol known as $\kappa$-Köhler theory [44, 45]. $D_{c}$ is the diameter of a particle at the critical supersaturation, and separates haze droplets, which are assumed to be in instantaneous equilibrium with the environmental saturation ratio, and cloud droplets, which are either growing or shrinking in response to the saturation ratio to which they are exposed. In all the experiments, size selected $\mathrm{NaCl}$ (dry diameter $=130 \mathrm{~nm}, \kappa=1.28$ ) were used as $\mathrm{CCN}$. At $T=20^{\circ} \mathrm{C}$ (which was the mean temperature maintained in all the experiments), the critical diameter of $130 \mathrm{~nm}$ diameter $\mathrm{NaCl}$ aerosol is $\approx 2 \mu \mathrm{m}$. Therefore, we considered all hydrometeors with a diameter greater than $2 \mu \mathrm{m}$ as cloud droplets. The size distribution of particles in the chamber was measured with a WELAS optical particle counter, in the range $0.6 \mu \mathrm{m}$ to $40 \mu \mathrm{m}$ diameter. The WELAS has a detection efficiency of approximately 1 down to $0.45 \mu \mathrm{m}$ diameter [46]. Panel (b) of figure 2.6 in section 2.6.2, shows a few examples of the size distribution haze and cloud droplets measured by the WELAS. 


\subsubsection{Cloud Droplet Residuals:}

The $\mathrm{CCN}$ upon which cloud droplets form can be isolated by evaporating the droplets, leaving residual particles. We collect cloud droplets using the PCVI, evaporate the water, then measure the dry aerosol size distribution using an SMPS. In the absence of collision-coalescence (which, to first order, is the case for the $\Pi$ chamber), the residuals are the size distribution of the particles that were activated. The PCVI collected cloud droplets which are larger than $\approx 4.5 \mu \mathrm{m}$. Since the PCVI has a low collection efficiency, the number concentration measured was corrected using the WELAS data. A multiplication factor, $M$, was introduced and defined as the ratio of measured number concentration of droplets larger than $\approx 4.5 \mu \mathrm{m}$, measured by the WELAS, to the number concentration

obtained from the PCVI, i.e. $M=\frac{n_{d}\left(D_{w} \geq 4.5\right)}{n_{r}}$, where $n_{r}$ is the number concentration of the residuals collected by the PCVI. Each bin in the distribution measured using the SMPS was then multiplied with this factor to obtain the final residual size distribution.

\subsubsection{Interstitial:}

As noted above, the critical diameter, $D_{c}$, separates those $C C N$ that have exceeded the peak in the Köhler curve and become cloud droplets from those that have not. In other words, if an aerosol does not overcome the threshold diameter (i.e. does not encounter the critical supersaturation), it remains as a haze particle and maintains an equilibrium size based on the environmental saturation ratio. The unactivated haze particles are called interstitials [47]. In our analysis, particles with diameter less than $2 \mu \mathrm{m}$ were considered interstitial aerosol. Haze 
droplets with diameters of $1 \mu \mathrm{m}$ or less were sampled using a stainless steel tube that projected into the chamber, and their diameter were measured using the SMPS (see Sec. 2.4 for details). Larger haze droplets could not be sampled in this manner. The number and (wet) size of those particles are determined using the WELAS. The number concentration of particles with wet diameters between 1 and 2 microns (measured with the WELAS) and the concentration measured with the SMPS were added to obtain the total number concentration of interstitial aerosol.

\subsubsection{Activated Fraction:}

The activated fraction, $F_{n}$, of aerosol particles can be considered as their activation efficiency [48]. It is defined as

$$
F_{n} \equiv \frac{n_{d}}{n_{d}+n_{i}}
$$

where, $n_{d}$ and $n_{i}$ are the concentration of cloud droplets and concentration of interstitial aerosols, respectively.

In all experiments, we set up the desired $\Delta \mathrm{T}$, to obtain a supersaturation and turbulent forcing. Size selected $\mathrm{NaCl}$ aerosol particles were introduced after the system reached thermodynamic steady state. All data reported here (unless otherwise noted) are for a second steady state condition, reached when the injection of aerosol particles was balanced by the removal of droplets through sedimentation. Figure 2.3 shows the number concentration of interstitial aerosol as a 


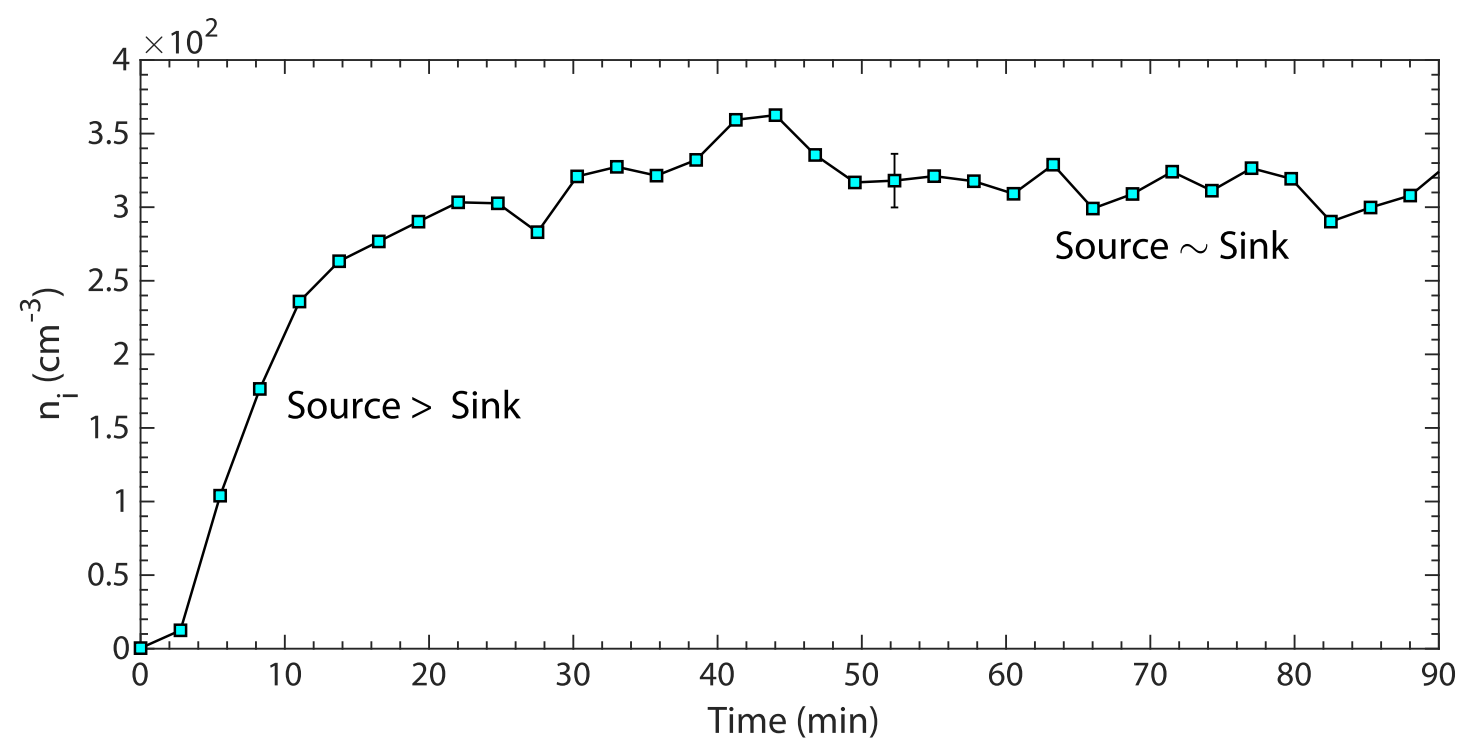

Figure 2.3: Time series of the number concentration of interstitial aerosols, showing an approach to steady-state. The uncertainty bar (at approximately the 53rd minute) shows the first standard deviation around the mean value of $n_{i}$ at the steady-state. At the start of the aerosol injection, the source (injection) was greater than the sink (activation). Aerosol-cloud steady state was reached when source was balanced by the sink and the concentration of interstitial reaches a plateau.

function of time where the plateau signifies the aerosol-cloud steady state condition.

\subsubsection{Turbulence in the Chamber}

The supersaturation in the experiments was created through turbulent mixing via Rayleigh-Bénard convection. The level of turbulence in the chamber was modified by adjusting the temperature difference between the top and bottom plates. However, in cloud-free or cloudy cases, there is a gradient in both temperature and water vapor concentration. The Rayleigh number was therefore calculated as [49]:

$$
R a_{v}=\frac{g \Delta T H^{3}}{T_{0} v \kappa_{T}}+\frac{g \epsilon \Delta q_{v} H^{3}}{v \kappa_{T}}
$$


where $\Delta T$ is the temperature difference between top and bottom plates, $T_{0}$ is the mean temperature, $\mathrm{g}$ is the gravitational acceleration, $v$ is the kinematic viscosity of the fluid, $H$ is the distance between the plates, $\kappa_{T}$ is the thermal diffusivity and $\epsilon=0.61$. Using this relation we can obtain an estimate of the turbulent kinetic energy dissipation rate, given by [50]:

$$
\langle\varepsilon\rangle=\frac{v^{3}}{H}(N u-1) R a_{v} \operatorname{Pr}^{-2}
$$

where, $R a_{v}$ is the Rayleigh number obtained from Eqn. 2.4, $P r$ is the Prandlt number $\left(\operatorname{Pr}=\frac{v}{k_{T}}\right)$ and $N u$ is the Nusselt number $\left(N u \simeq 0.27 \mathrm{Pr}^{-1 / 7} \mathrm{Ra}^{2 / 7}\right)$.

\subsection{Results and Discussion}

Our focus was to explore aerosol activation and its dependence on the cloudfree, $S_{0}$, and in-cloud, $S$, saturation ratios in a turbulent environment. In our experiments, $S_{0}$ is the forcing, and the quantity over which we have the most direct experimental control. The analogous variable from adiabatic parcel theory is the rate at which supersaturation increases in the absence of droplets, frequently written as $\alpha w$ where $\alpha$ is a function of $T$ and $w$ is the updraft. In the chamber, when $\mathrm{CCN}$ are injected, the initial saturation ratio, $S_{0}$, is reduced as water condenses. In adiabatic parcel theory, this value, $S$, is analogous to the value of supersaturation in the parcel once $\alpha w$ and the sink are both considered. In essence, $S$ is the response of the system considering all sources and sinks. (See [42] for a more complete discussion of supersaturation in an adiabatic parcel.) We alter $S_{0}$ by changing $\Delta T$ and the saturation ratio at the lower boundary. We 
have limited control over $S$, but by holding $S_{0}$ constant and varying the injection rate of $\mathrm{CCN}$, we can change the sink in the system, thus changing $S$.

Through the laboratory measurements, we examined:

1) The effect of the mean forcing, $S_{0}$, and the turbulent fluctuations about the mean, $\sigma_{S_{0}}$, on aerosol partitioning between the interstitials and cloud droplets, and

2) The effect of in-cloud supersaturation $\left(S\right.$ and $\left.\sigma_{S}\right)$ on the activated fraction.

Since the experiments were performed in a controlled environment, the chemical composition and the size of the aerosol are constant in all the experiments, isolating the effects of the parameters listed above. We used size selected 130 $\mathrm{nm} \mathrm{NaCl}$ as $\mathrm{CCN}$ for all the experiments. The number concentration of the input aerosols was also kept constant, with the exception of experiments designed to determine the effect of the number concentration of aerosol on the activation process.

\subsubsection{Effect of Fluctuations on Aerosol Partitioning}

Our first result is that fluctuations in the supersaturation blur the relationship between size, chemical composition, and activation. In the traditional formulation, where all particles in a parcel are assumed to be exposed to the same, uniform supersaturation, activation can be predicted by the particle's size and chemical composition [27, 28]. In a turbulent environment, a particle's critical 


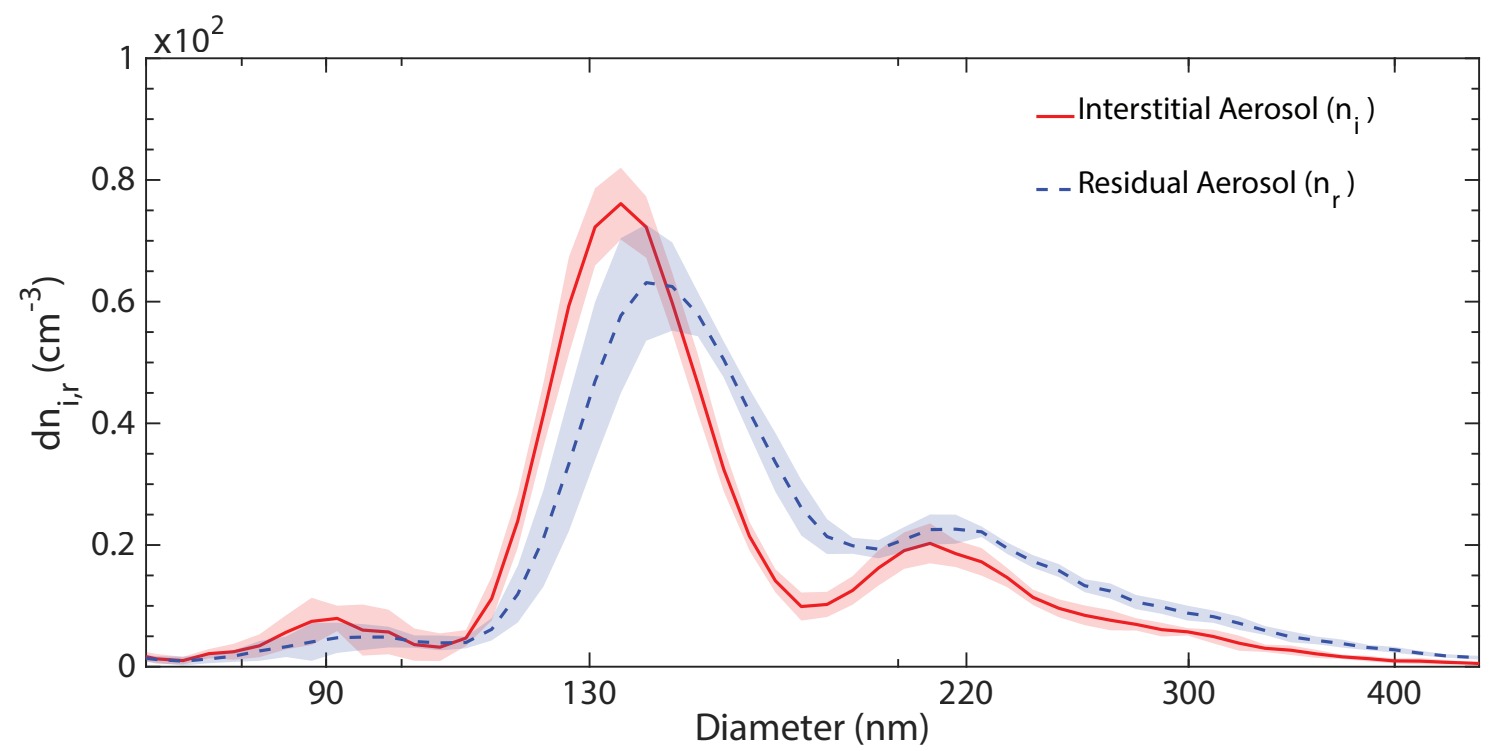

Figure 2.4: Size distributions of interstitial (solid, red) and residual (dashed, blue) aerosol for a turbulent, cloudy condition in the chamber. The shaded region indicates one standard deviation. $\mathrm{NaCl}$ was size-selected at $130 \mathrm{~nm}$ and injected at a constant rate of $\approx 170 \mathrm{~cm}^{-3} \mathrm{~min}^{-1}$. The peak at $\approx 210 \mathrm{~nm}$ is from doubly charged particles, which have the same electrical mobility as singly charged $130 \mathrm{~nm}$ particles. The peak at $\approx 90 \mathrm{~nm}$ is from particles that were singly charged when they were injected into the chamber, but doubly charged when they were measured with the SMPS after being sampled from the chamber. The distributions shown here are the average of approximately 10 distributions collected over $30 \mathrm{~min}$ in steady state conditions. The size cut used for the PCVI was $4.5 \mu \mathrm{m}$ diameter. $\Delta T=18 \mathrm{~K}$ for these experiments.

supersaturation is still a function of those two parameters, but not all particles will be exposed to the same supersaturation. Figure 2.4, a plot of the size distribution of interstitial and residual aerosol, shows this clearly because the quasimonodisperse CCN are seen in both interstitial and residual distributions.

For this experiment, there was a temperature difference of $18 \mathrm{~K}$ between the plates in the chamber, corresponding to $\mathrm{Ra}=2.3 \times 10^{9}$ and $\langle\varepsilon\rangle=2 \times 10^{-3} \mathrm{~m}^{2} \mathrm{~s}^{-3}$. The almost complete overlap of the two distributions shows that particles of the same critical supersaturation were both activated and remained as interstitial. Because of the spatial and temporal heterogeneity of the saturation ratio in the 
chamber, there were injected particles that did not encounter a supersaturation great enough for activation before being sampled. These are the interstitials (It is possible that some aerosol that are sampled as interstitial have activated but then encountered a subsaturated region and deactivated. We think that this is unlikely here. This issue is addressed below.) There are also particles that were injected into the chamber that did encounter a sufficiently large supersaturation, became a cloud droplet, and were sampled with the PCVI. These are the residuals. The fact that particles of the same size and chemical composition, and thus the same critical supersaturation, are seen in both interstitial and residual distributions is an unambiguous demonstration that fluctuations from turbulence blur the concept of a single peak supersaturation in a parcel and the clear dividing line between activated and interstitial based on that peak supersaturation.

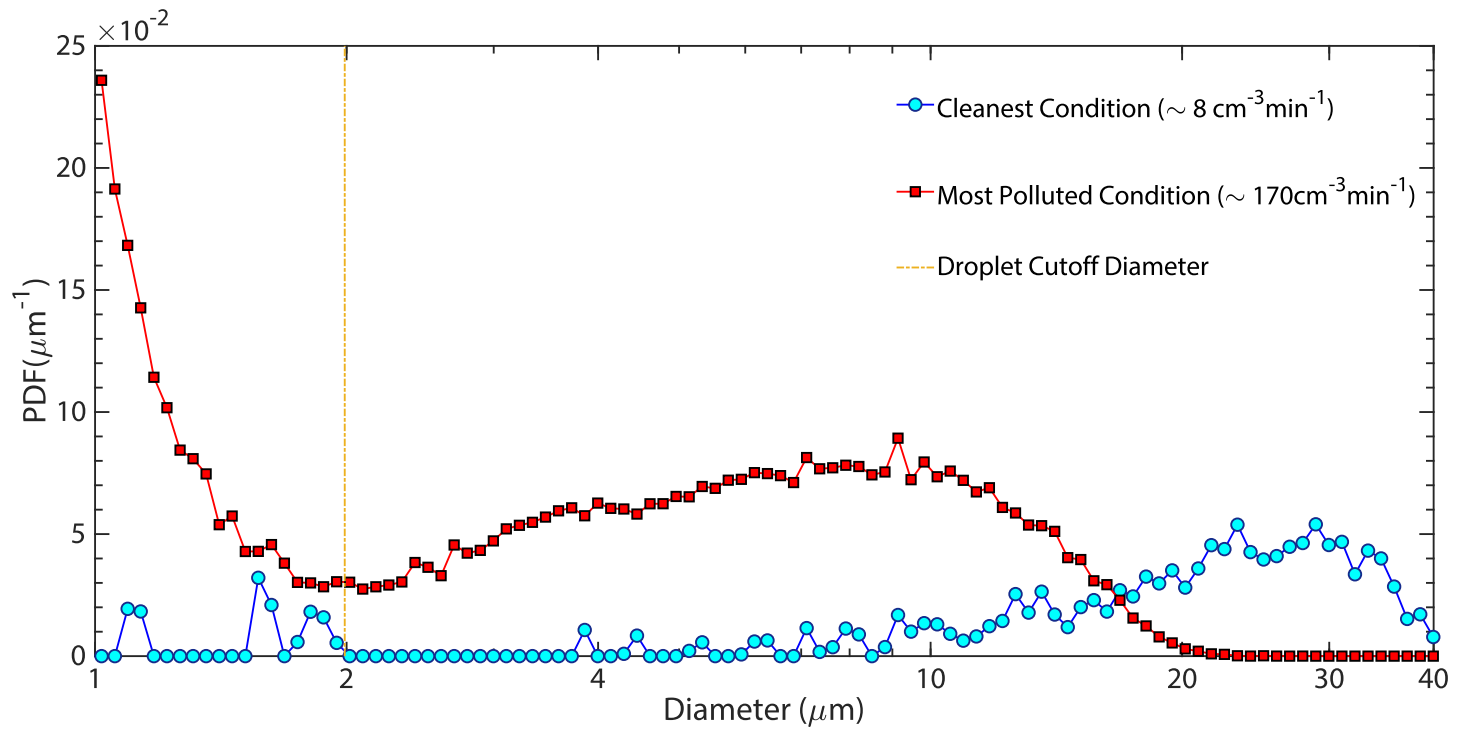

Figure 2.5: The size distribution of haze and cloud droplets measured from the chamber using the WELAS. Clean and polluted conditions are shown (blue circles and red squares respectively). The orange dotted line corresponds to the the cutoff diameter considered to delineate interstitials and droplets. The numbers in the parentheses indicate the corresponding aerosol injection rate. 
Simultaneous coexistence of particles with the same critical supersaturation as interstitials and as cloud droplets is also evident in Figure 2.5, a plot of the particle probability density function from two different experiments in the chamber $(\Delta \mathrm{T}=18 \mathrm{~K})$. In both cases, size selected aerosol were injected, but the injection rate was varied to create clean to polluted cloud conditions. In the cleanest conditions (shown with blue circles), the steady state supersaturation in the chamber was high, activating essentially all the particles. The activated droplets quickly grew to diameters exceeding $10 \mu \mathrm{m}$, where they settled out of the chamber. In contrast, for higher injection rates (shown with red squares), where competition for water vapor is greater and the in-cloud supersaturation is lower, there are two clearly identifiable populations of interstitial aerosol particles and activated droplets. (The vertical dashed line in the figure shows the critical diameter for $130 \mathrm{~nm}$ diameter $\mathrm{NaCl}$.) The fact that there is a reservoir of both unactivated particles and a population of droplets in the chamber, is the evidence that there are significant spatial and temporal variations of the saturation ratio in the chamber, activating some particles and leaving some as interstitial. Fluctuations in the turbulent environment destroy the one-to-one correspondence between activation and chemical composition and size.

There are limited instances of concurrent measurements of interstitial aerosol and cloud droplet residuals, due in part to the difficulty in measuring the interstitial distribution from an aircraft. One case in which the two were observed, performed at the mountaintop observatory at Jungfraujoch, showed particles of the same size present as both interstitial and cloud droplet residual [51]. The investigators in that study attributed it to differing chemical compositions. 
Another measurement at Jungfraujoch, performed by [52], focused on aerosolpartitioning in mixed phase clouds. The size distribution of both activated and interstitial aerosol were measured using a total inlet, and only interstitials separately using an SMPS. The comparison between the total inlet and the interstitial showed coexistence of interstitials and activated aerosols of same size in both warm and mixed phase clouds. This overlapping of particles was attributed to the heterogeneity in chemical composition and evaporation of the droplets due to the presence of ice crystals. [53] measured the in-cloud residuals and below-cloud aerosol of stratocumulus clouds over the southeast Pacific Ocean to determine which particle sizes acted as CCN for the clouds they investigated. They found particles of the same size in both interstitial and residual, which was also shown in LES modeling of the cases. It should be noted that in both cases, the distributions were averaged over several seconds of flight time such the particles shown in the same distribution may have had quite different thermodynamic histories. The first experimental study, to our knowledge, showing particles present as both cloud droplets and interstitial solely as the result of turbulence was [13]. Polydisperse aerosol was used as the input to the $\Pi$ Chamber in that case. More recently, results from another type of turbulent cloud chamber have shown co-existence of droplets and interstitial for monodisperse aerosol particles of a single chemical composition [54].

The partitioning of aerosol without a clear separation of cloud droplets and haze droplets (for the same size and chemical composition) constitutes the importance of turbulence in the activation process. However, to distinguish the effect of how the initial (i.e., no-cloud) mean saturation ratio, $S_{0}$, and turbulent fluctuations 
about that value, $\sigma_{S_{0}}$, affect the activation rate independently, we modified the chamber's boundary condition, as discussed in Section 2.5 above. Using the modified setup, we investigated the effect of mean saturation ratio by keeping the same $\Delta \mathrm{T}$, which corresponds to the same turbulent forcing, but reducing the vapor pressure at the bottom boundary, thus reducing the mean saturation ratio. We also investigated the effect of fluctuations by holding the mean saturation ratio constant, while increasing $\Delta \mathrm{T}$ (as illustrated in Fig. 2.2). We discuss the effect of the mean saturation ratio first.

\subsubsection{The Effect of Mean Forcing, $S_{0}$}

As outlined at the beginning of the section, one of the goals in these experiments was to quantify the effect of the mean forcing (i.e. $S_{0}$ ) and the fluctuations around it. To that end, panel a of Figure 2.6 is a plot of the activated fraction, $F_{n}$, as a function of the mole fraction of water, $\chi_{\mathrm{H}_{2} \mathrm{O}}$ at the bottom boundary of the chamber, which is a proxy for $S_{0}$. (See Section 2.5 for details of the boundary conditions.) In these experiments, $\Delta T=20 \mathrm{~K}$ and aerosol injection was constant at $\sim 70 \mathrm{~cm}^{-3} \mathrm{~min}^{-1}$, so adding salt to the bottom boundary (i.e. decreasing $\chi_{\mathrm{H}_{2} \mathrm{O}}$ ) had the effect of reducing the forcing while holding the fluctuations constant. Note that the Rayleigh number is dominated by $\Delta \mathrm{T}$, so a small reduction in vapor pressure at the bottom boundary as we added salt did not reduce the overall turbulent fluctuations significantly. (The Rayleigh number given by eqn. 2.4 is dominated by the term proportional to $\Delta T$; the correction afforded by term proportional to $\Delta q_{v}$ is an order of magnitude lower.) We have plotted $\chi_{\mathrm{H}_{2} \mathrm{O}}$, not 
$S_{0}$, because our determination of the absolute value of the saturation ratio in the chamber does not have sufficient accuracy.

There is a monotonic decrease in $F_{n}$ as $\chi_{\mathrm{H}_{2} \mathrm{O}}$, and thus $S_{0}$, decreases. At the highest supersaturation $\left(\chi_{\mathrm{H}_{2} \mathrm{O}}=1\right)$, the majority of aerosol particles injected into the chamber become cloud droplets. As $S_{0}$ is reduced, fewer of the aerosol particles encounter a supersaturation greater than their critical value. At $\chi \approx 0.95$, $F_{n}$ drops below 0.5 ; the rate of decrease in $F_{n}$ changes noticeably, suggesting a change in the mechanism.

To see this more clearly, we examine the size distributions of particles in the chamber (droplets and interstitials), shown in Panel b of Fig. 2.6. Distributions from three different values of $S_{0}$ are plotted, corresponding to high, medium, and low $F_{n}$, labeled I, II, and III in Panel a. At point I, a high value of $F_{n}$, there are clearly two modes in the distribution, corresponding to interstitial aerosol and cloud droplets (the orange line indicates the critical diameter for $130 \mathrm{~nm}$ $\mathrm{NaCl}$ ), but there are more droplets than interstitials. At point II, the minimum between the two modes is also clearly evident, but the proportion of interstitials and droplets has reversed. At point III, $F_{n}$ has essentially gone to zero; most of the particles in the chamber are interstitial, with a very small number of droplets.

The trend seen in Figure 2.6 is analogous to the three regimes of $\mathrm{CCN}$ activation described by [2]: aerosol-limited, updraft-limited, and transitional. The regimes were proposed based on the peak supersaturation and fraction of aerosol activated in a cloud parcel model, where updraft (i.e. adiabatic cooling) is the source of the supersaturation and growing cloud droplets are the sink. (See [55] 
(a)
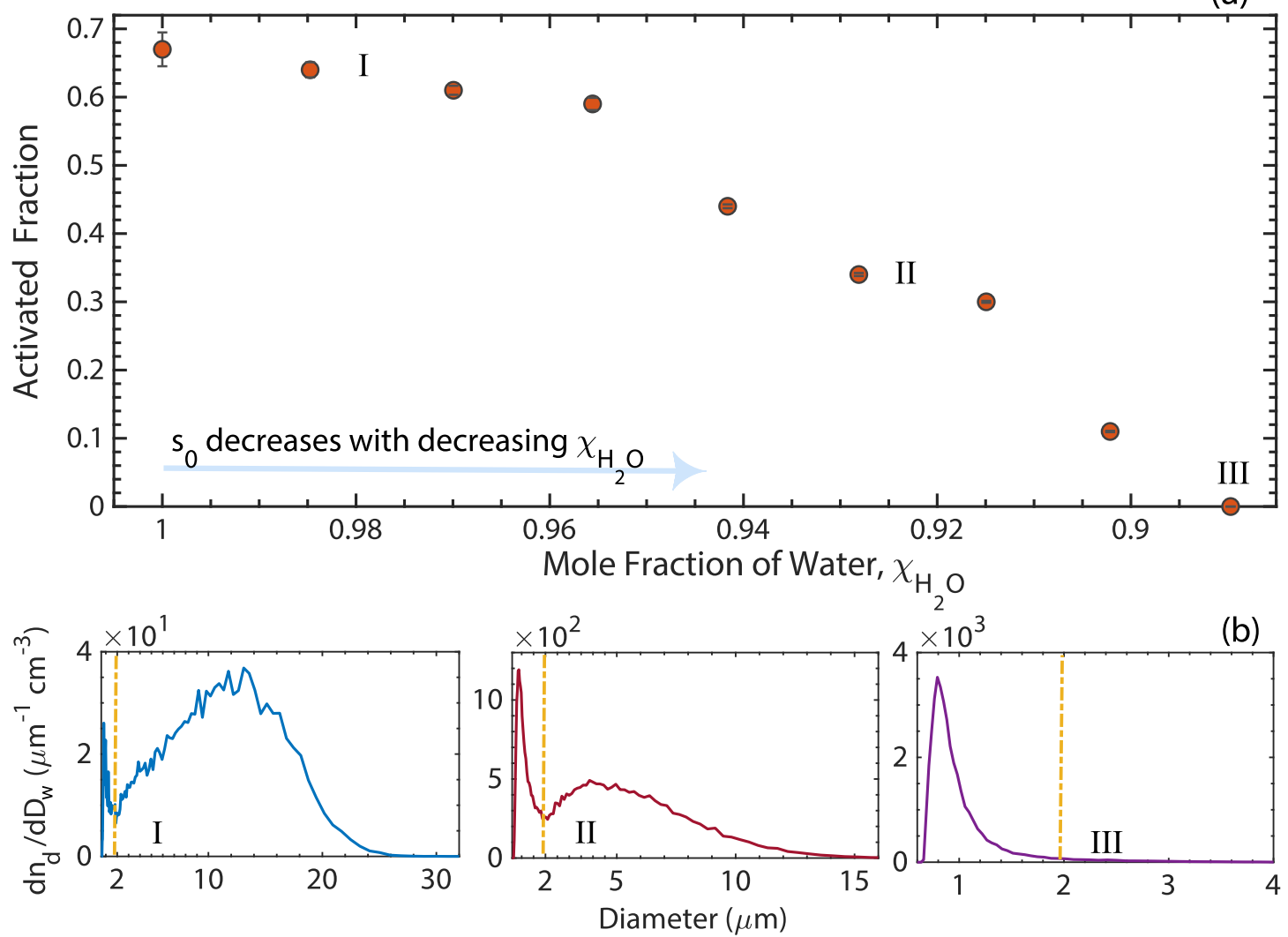

Figure 2.6: Effect of $S_{0}$ on activation with fluctuations held constant, showing (a) activated fraction as a function of the mole fraction of water $\left(\chi_{\mathrm{H}_{2} \mathrm{O}}\right)$ at the bottom boundary, and $(b)$ size distributions of interstitial aerosol and droplets for three different cases. $\chi_{\mathrm{H}_{2} \mathrm{O}}$ gives an estimation of the relative change in forcing, $S_{0}$, in the system. As $\chi_{\mathrm{H}_{2} \mathrm{O}}$ decreases $S_{0}$ also decreases. All these experiments had the same $\Delta T$ and the same injection rate of $\mathrm{NaCl}$ aerosol particles. The only variable was $S_{0}$. Thus, in panel (a), the largest activated fraction was achieved when we have only fresh water at the bottom, i.e., at the highest $S_{0}$. No activation was observed when the bottom boundary had the highest amount of salt, i.e., at lowest $S_{0}$. Size distributions shown in blue, red, and purple correspond to the three points marked I, II, and III in panel (a). The orange dashed line, showing the critical diameter of $130 \mathrm{~nm} \mathrm{NaCl}$ aerosol $\left(D_{c}\right)$, is the demarcation between the cloud and the haze droplets.

for an extension of these ideas, covering relative dispersion.) In our experiments, $S_{0}$ is the source term. Panel a of Figure 2.6 shows at least two distinct regimes achieved in the controlled laboratory environment. The cases with higher activated fraction could be considered as the transitional regime while the final plot 
in Panel b corresponds to the updraft limited regime, which is low supersaturation to the point of subsaturation.

Though there are similarities between the regimes suggested by [2] and the regimes achieved in the $\Pi$ chamber, the fundamental difference is the inclusion of fluctuations. At a given altitude or moment in time, the parcel model is characterized by a single value of supersaturation. A particle in the parcel with a given size and chemical composition either will or will not activate, depending on the relationship between the particle's critical supersaturation and the supersaturation in the parcel. As noted by [3] and discussed more extensively there, in our experiments, there are fluctuations around $S_{0}$. We characterize the regimes in the chamber based on the relative values of $S_{0}$ and $\sigma_{S_{0}}$ and the critical saturation ratio of the particles, $S_{c}$. There is a regime where $S_{0}$ is dominant, a regime where $\sigma_{S_{0}}$ is dominant, and an intermediate regime between those two. In the mean dominated regime, $S_{0}$ is significantly greater than $S_{c}$ for the particle. One of the important characteristics of this regime is that it exhibits a dominant mode in the particle distribution, corresponding to the droplets. Essentially, $F_{n}$ is unity. The saturation ratio is always large enough to activate all aerosol particles, even considering fluctuations. We do not see this regime in the experiments shown in figure 2.6, though the cleanest case shown in Figure 2.5 exhibits this behavior. In the intermediate or fluctuation-influenced regime, $S_{0}>S_{c}$, but the distribution of the saturation ratio in the chamber is such that a significant portion of it is less than $S_{c}$. The particles that encounter these subcritical values remained as interstitials. $F_{n}<1$ and two distinctive modes, corresponding to haze and cloud droplets with a distinctive minimum between the two, characterize this 
regime. Since the distributions of the first two cases (I and II) show a distinctive minimum and modes of both interstitial and cloud droplets, these two cases fit in the fluctuation influenced regime. Lastly, a fluctuation-dominated regime can be characterized by a dominant mode of interstitials without any distinctive minimum. In this regime $S_{0}$ is lower than $S_{c}$ and aerosol only activate through positive fluctuations in the saturation ratio. $F_{n}$ is expected to be very low as a result. Point III in Figure 2.6 is in this regime. (For a more comprehensive discussion of these regimes and an underlying theory, see [3].) We return to the discussion of the fluctuation-dominated regime in Section 2.6.3.

\subsubsection{Effect of Fluctuations in Forcing, $\sigma_{s_{0}}$}

In the previous section, we describe experiments in which we explored the role of the mean forcing in the partitioning between activated and unactivated aerosol. We now turn to the role of fluctuations. To isolate the effects of fluctuations about the mean in the forcing, we reconfigured our experimental setup to hold the mean saturation ratio constant but vary the level of turbulence, and thus the fluctuations. We considered two base cases. Case A had a low $S_{0}$ with $\Delta T=6 \mathrm{~K}\left(S_{0,6}\right)$, and case B had higher $S_{0}$ at $\Delta T=9 \mathrm{~K}\left(S_{0,9}\right)$. To hold $S_{0}$ constant, but change $\sigma_{S_{0}}$, we added salt to the bottom boundary, while simultaneously changing $\Delta T$. Adding salt decreases $S_{0}$, but an increase in $\Delta T$ increases it. The two changes together can be used to match $S_{0}$ at two values of $\Delta T$ (as illustrated in Fig. 2.2. . The higher value of $\Delta T$ will have the higher $\sigma_{S_{0}}$. (See Section 2.5 for further details.) $\mathrm{CCN}$ of the same size and chemical composition (130 nm 
diameter $\mathrm{NaCl}$ ) were used in all the experiments with a constant injection rate of approximately $33 \mathrm{~cm}^{-3} \mathrm{~min}^{-1}$.

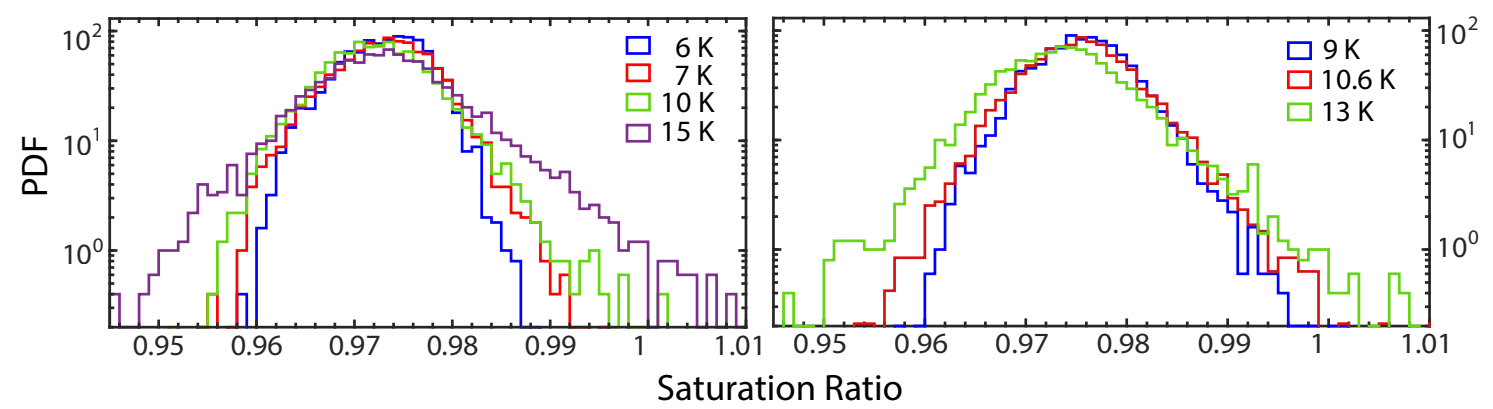

Figure 2.7: Probability density functions of $S_{0}$ for case A (left) and B (right). Note that the mean is constant, but that the width of the PDFs increase with $\Delta T$. There is an offset between the measured $S_{0}$ and actual values of $S_{0}$ in the system. However, the relative changes among different cases as well as the measured $\sigma_{S_{0}}$ are reliable [1].

Figure 2.7 shows the probability distribution functions of $S_{0}$ for cases $\mathrm{A}$ and $\mathrm{B}$. In case $\mathrm{A}$ (left panel), the base is for $\Delta T$ of $6 \mathrm{~K}$. Subsequent experiments at $\Delta T=$ 7,10 , and $15 \mathrm{~K}$ had the same mean saturation ratio, $S_{0,6}$, but larger $\sigma_{S_{0}}$. For $\Delta T=$ $9 \mathrm{~K}$, experiments at 10.6 and $13 \mathrm{~K}$ had the same mean saturation ratio, $S_{0,9}$ (right panel). There is an evident change in the width of the PDFs at the different $\Delta \mathrm{T}$. As discussed in section 2.4, $S_{0}$ measured using LI-COR and RTDs may have an offset from the actual value. However, the relative changes in $S_{0}$ and measured $\sigma_{S_{0}}$ are reliable [1]. The activated fractions, $F_{n}$, for all these cases are shown as a function of $\Delta \mathrm{T}$ in Figure 2.8. The blue and red triangles represent $F_{n}$ for cases $\mathrm{A}$ and $\mathrm{B}$ respectively. The second $\mathrm{x}$ axis shows the equivalent turbulent kinetic energy dissipation rate, $\langle\varepsilon\rangle$, calculated using equation 2.5. $\varepsilon$ calculated for the cloud chamber is comparable to those seen in stratocumulus clouds [56, 57, 58, 59], which is in the order of $10^{-3} \mathrm{~m}^{2} \mathrm{~s}^{-3}$. This dissipation rate is sufficiently weak that inertial clustering and its influence on supersaturation fluctuations can be 


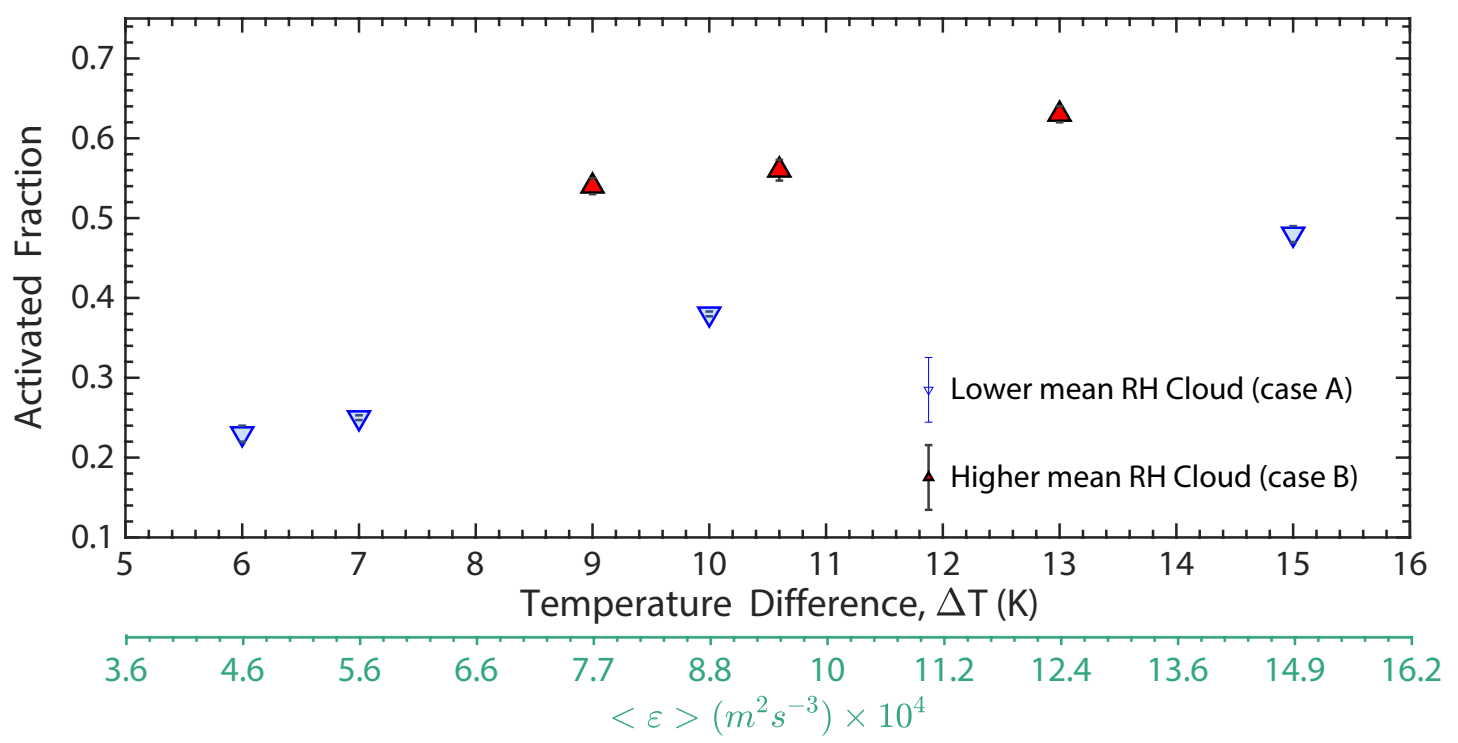

Figure 2.8: Activated fraction as a function of $\Delta \mathrm{T}$ in the chamber. Blue (downward pointing) and red (upward pointing) triangles represent $F_{n}$ for cases $\mathrm{A}$ and $\mathrm{B}$ respectively. The mean saturation ratio was held constant for each case, but the fluctuations increased, as shown in Figure 2.7. The secondary $x$ axis shows the turbulence energy dissipation rate corresponding to $\Delta \mathrm{T}$. Since case A had a lower initial saturation ratio, it also has a lower initial activated fraction..

neglected. Specifically, droplet Stokes numbers are of order $10^{-2}$ and below and prior work has demonstrated that in that regime, combined with the importance of gravitational settling, inertial clustering is extremely weak [60, 61, 62].

It is evident from the figure that $F_{n}$ increases with increasing $\Delta T$ (therefore, increasing $\langle\varepsilon>$ ), corresponding to increasing fluctuations in the saturation ratio. Though the mean saturation ratio is the same for each case, an increase in the fluctuations exposed a higher fraction of the aerosol in the chamber to a saturation ratio greater than their critical value.

To further illustrate the influence of fluctuations, we consider the integral diameter, $I=n_{d} \overline{D_{w}}$, defined as part of Eqn 2.2. The integral diameter is a measure of 
the total sink for water vapor in a cloud. The upper panel of Figure 2.9 shows the integral diameter for these experiments as a function of the temperature difference between the plates in the chamber. An immediate conclusion from this plot is that fluctuations are driving changes in $I . S_{0}$, the forcing, is a constant for each series of experiments, yet $I$ is changing with $\Delta T$, which forces the fluctuations. The two lower panels in Figure 2.9 illustrate this even more clearly. For
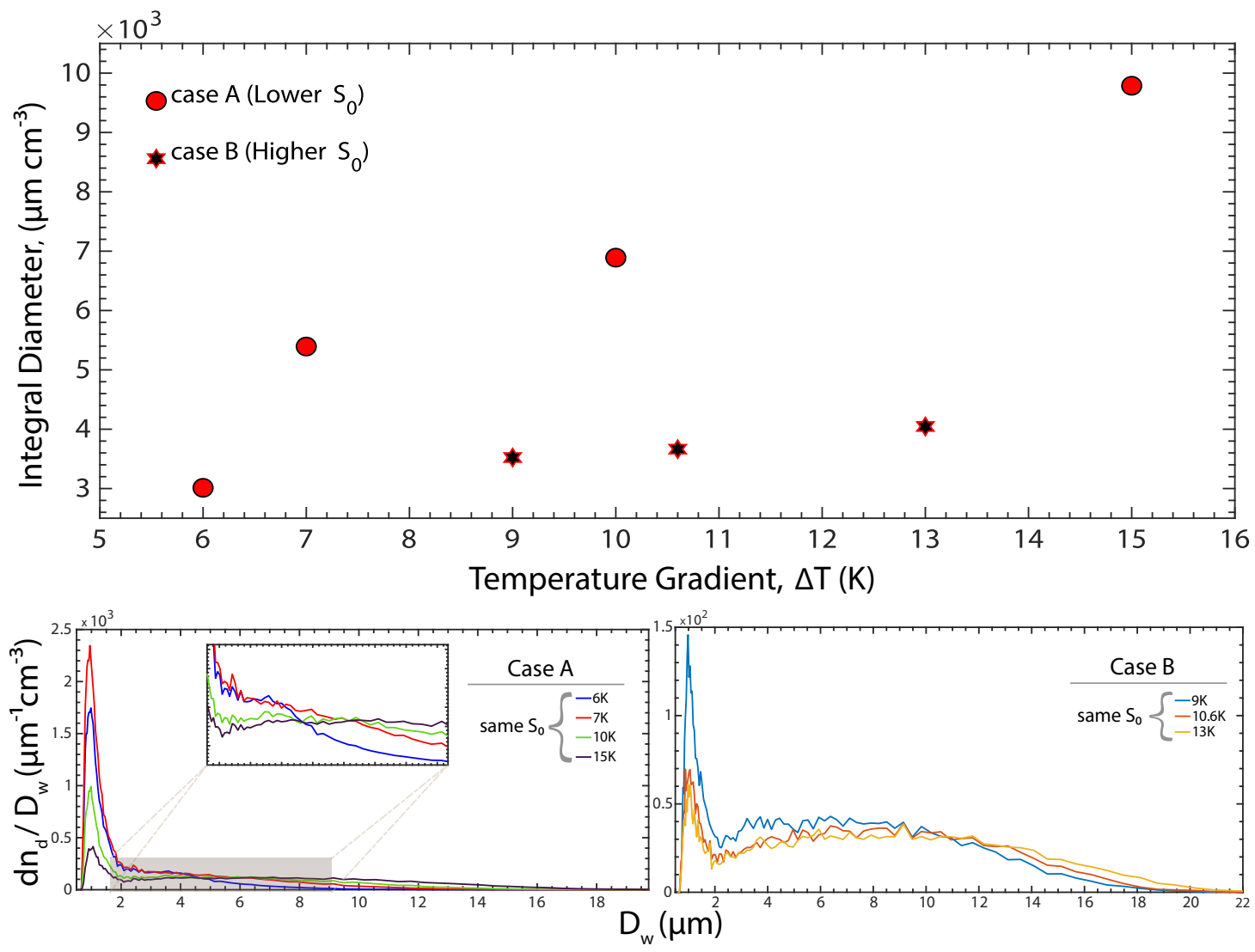

Figure 2.9: Figure showing relationships between integral diameter and fluctuations in the chamber. Top: integral diameter as a function $\Delta T$ for both case A and B. Bottom left: Size distributions of particles in the chamber for case A. Bottom right: Size distributions of particles in the chamber for case B. Note that a constant $S_{0}$ was maintained for all the gradients in case $\mathrm{A}\left(S_{0,6}\right)$, and all the gradients in case $\mathrm{B}\left(S_{0,9}\right)$. Only $\sigma_{S_{0}}$ was varied in these cases (see Figure 2.7). Note the differences in the number concentrations of particles in the chamber for the two cases. Though the injection rate is the same for all cases, the residence time of particles in the chamber changes, resulting in a different concentration of particles in the chamber. 
case $\mathrm{A}$, the absence of a minimum in the droplet size distribution at $\Delta T=6$ and $7 \mathrm{~K}$ indicate that the system is subsaturated in the mean (See [3] for a more complete discussion of this point.) The integral radius is nonzero only because fluctuations in the chamber produce local regions of supersaturation where cloud droplets form and grow. As fluctuations increase further, more cloud droplets with larger diameters were observed.

The size distributions in the right panel of Figure 2.9 are in the fluctuationinfluenced regime as both interstitial and cloud droplet modes are visible, with a clear minimum separating them. The presence of the droplet mode indicates that the mean saturation ratio of the base case $(\Delta T=9 \mathrm{~K})$ is higher than case $A$. With increasing fluctuations, more droplets are activated and droplets can grow slightly larger resulting in a larger integral diameter. The increase in the integral diameter with $\Delta T$ is not as large in this case as in the previous case because the integral diameter is already fairly large.

\subsubsection{Effect of In-cloud Saturation Ratio, $S$ and $\sigma_{S}$}

Having considered the effects of the initial forcing, $S_{0}$, and the initial fluctuations, $\sigma_{S_{0}}$, we turn to an explicit investigation of the in-cloud saturation ratio, $S$. While $S_{0}$ is determined by the temperature difference between the plates in the chamber, $S$ is a result of both the source and the sink. As noted above, $S_{0}$ can be considered the source term, while $S$ is the overall response of the system. In very clean conditions, $S \approx S_{0}$ because the sink of only a few growing cloud droplets is insufficient to deplete the vapor concentration. In contrast, in polluted conditions, $S \approx 1$ because a large number of droplets constitute a large sink. 


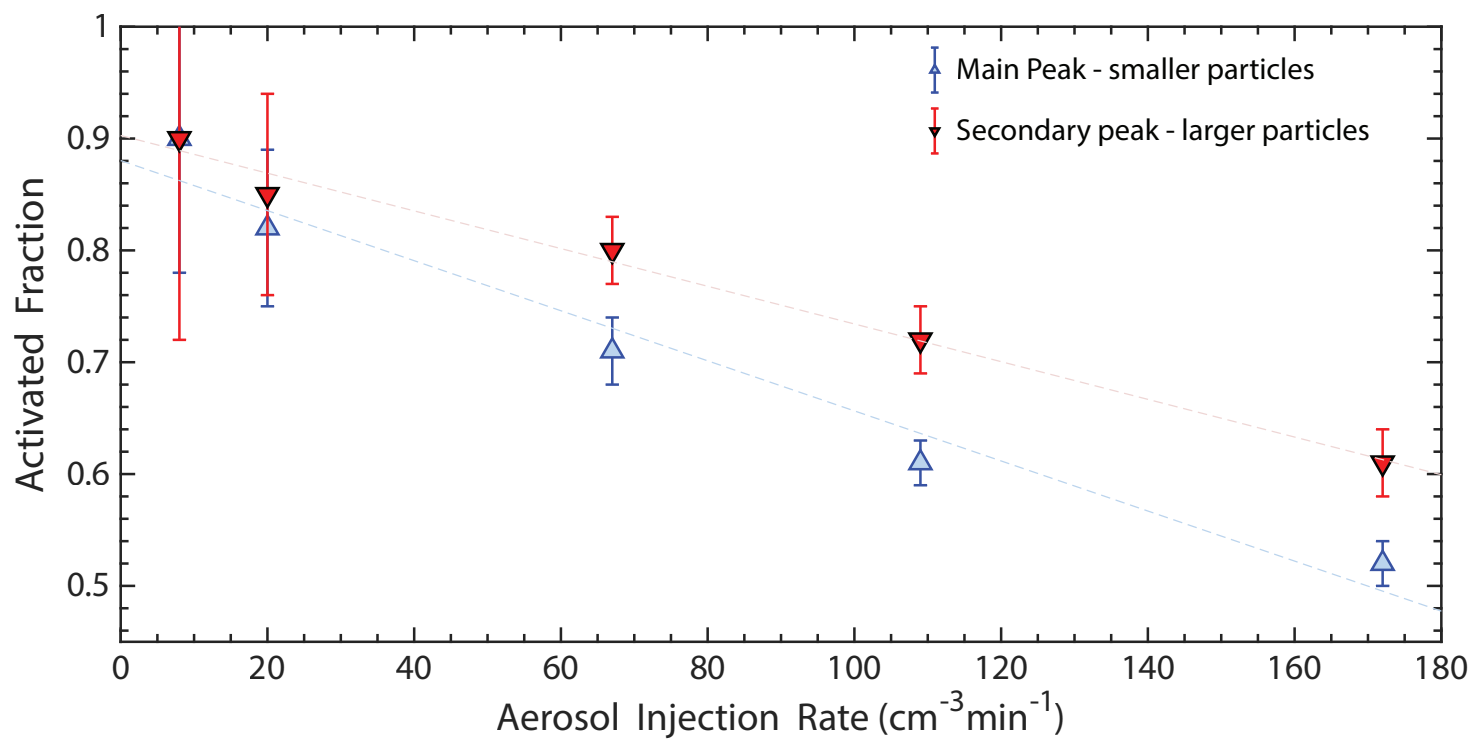

Figure 2.10: Activated fraction, $F_{n}$, as a function of aerosol injection rate. Upward pointing triangles (blue) and downward pointing triangles (red) correspond to the smaller (singly charged, $130 \mathrm{~nm}$ ) and larger doubly charged particles (220nm, doubly charged) aerosol respectively. The lines are the linear fits calculated for each mode. The temperature difference in the chamber was held at $18 \mathrm{~K}$; thus the forcing, $S_{0}$, was constant in these experiments. Differences occur because the in-cloud saturation ratio, $S$, is changing.

To explore the response of $F_{n}$ to $S$, we performed experiments where the only variation in conditions was the rate of injection of aerosol into the chamber. The initial forcing was held constant with a $\Delta T$ of $18 \mathrm{~K}$ between the plates. The lowest injection rate was 8 particles $\mathrm{cm}^{-3} \mathrm{~min}^{-1}$; the highest injection rate was 172 particles $\mathrm{cm}^{-3} \mathrm{~min}^{-1}$. Activated fraction as a function of injection rate is shown in Figure 2.10. As noted above, all experiments were conducted using aerosol particles size selected with a DMA. Here, we use the fact that smaller, singly charged particles have the same electrical mobility as larger, doubly charged particles; both sizes are "selected" by the DMA. We use the cloud droplet residuals, measured using a PCVI, and the interstitials to calculate the activated fraction for both modes separately. (Note that because they are different sizes, the 


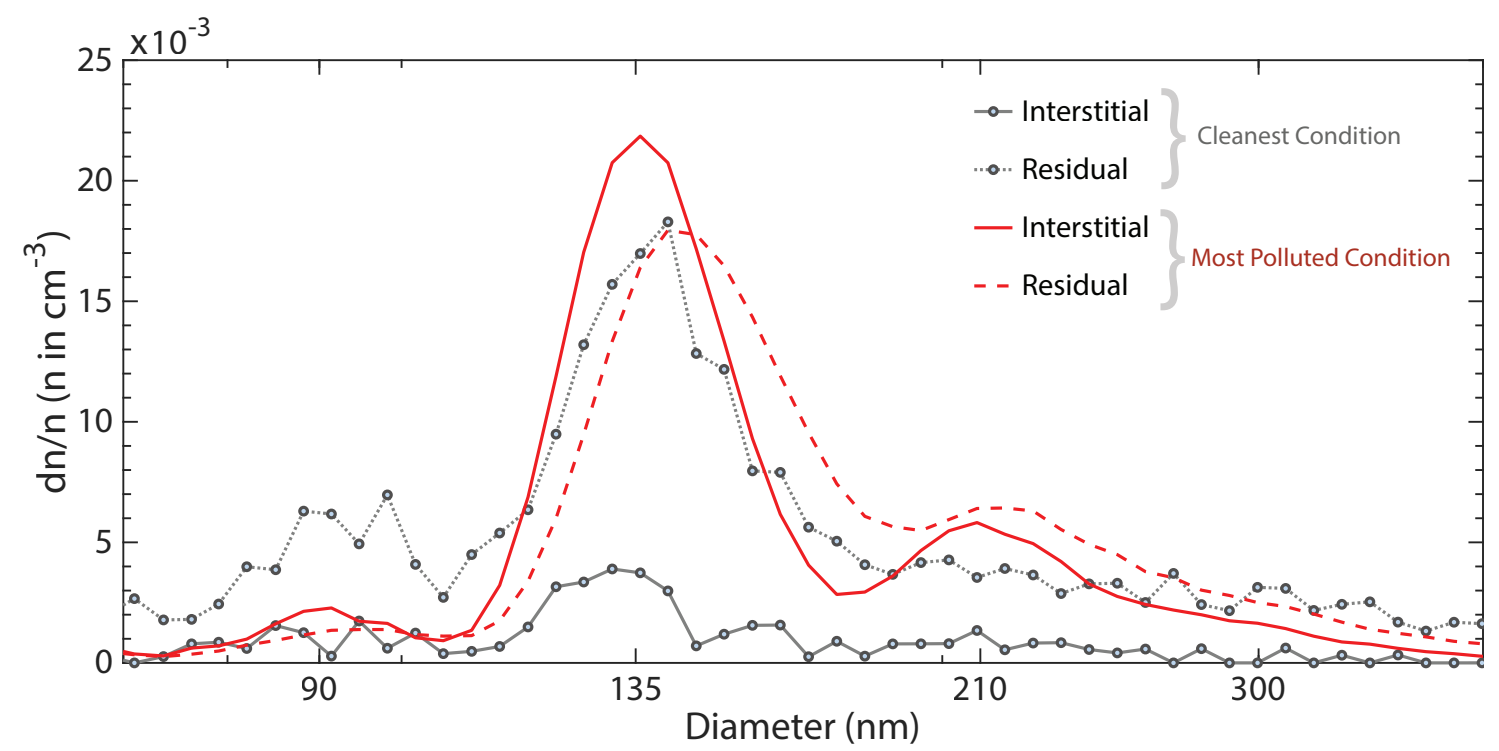

Figure 2.11: Normalized size distribution of interstitial (solid lines) and cloud droplet residuals (dashed lines) from the cleanest (grey lines) and most polluted (red lines) cases shown in Figure 2.10. The distributions are normalized by the total number concentration of aerosols in each case. In the cleanest condition, there was almost no interstitial, with nearly all the aerosols found in residuals. However, in the polluted condition, the scenario was reversed, and more interstitials were found than residuals.

two modes will have different critical supersaturations.) $F_{n}$ is observed to decrease with increasing injection rate, because competition among cloud droplets for available water vapor increases. Though the trend is not dramatic, the larger particles are more likely to become cloud droplets with decreasing $S$, even in a turbulent environment. This trend is also apparent in Figure 2.11, a plot of the size distributions of the cloud droplet residuals and the interstitials from the cleanest and most polluted cases shown in Figure 2.10. In the cleanest case, a comparison of the size distributions of the interstitials and droplet residuals shows that the supersaturation in the chamber, even when a cloud had formed was high enough to activate essentially all of the aerosol particles injected; i.e., there are only a few of the smallest particles seen in the distribution of residuals. 
In the polluted case, approximately half of the particles are unactivated; furthermore the distributions of the interstitials and cloud droplet residuals are quite similar and reflective of the distribution injected into the chamber. (The slight shift between the interstitials and residuals is the object of ongoing experimentation.) In both cases, however, the effect of fluctuations about $S$ are evident.

\subsubsection{An Expression for Activated Fraction, $F_{n}$}

Based on the results shown in the preceding sections and the context of the framework presented in [3], we propose a complementary error function as a simple model for the fraction of activated aerosol, shown in equation 2.6 and illustrated in Figure 2.12. In this model, $F_{n}$, depends on a particle's critical saturation ratio, $S_{c}$, the mean saturation ratio in the environment, $S$, and the width of the distribution of $S_{e n v}, \sigma_{S}$. For monodisperse particles with the same chemi-

cal composition as we have used in our experiments, $S_{c}$ is constant, represented by the red vertical dashed line in the figure. We assume that the profile of saturation ratio follows a Gaussian distribution, which measurements in the atmosphere suggest is valid (e.g. [59]). The activated fraction in this system is then the probability for a particle to encounter a saturation ratio greater than its critical value,

$$
F_{n}\left(S_{c}\right)=\frac{1}{2} \operatorname{erfc}\left[\frac{S_{c}-S}{\sqrt{2} \sigma_{s}}\right]
$$

The function and figure show that the activated fraction responds to both $S$ and $\sigma_{S}$. A change in $S$ or $\sigma_{S}$ alone can give the same activated fraction. This equation 
has some other notable properties. For example, half of the aerosol will activate if $S$ coincides with $S_{c}$ of the aerosol. The distribution of saturation ratios, characterized by $\sigma_{S}$, will aid both in activating and deactivating particles. From this

perspective and referring back to Figure 2.10, equation 2.6 suggests that in the most polluted condition $S \approx 1.0008$, which is the critical supersaturation for a $130 \mathrm{~nm}$ diameter $\mathrm{NaCl}$ aerosol particle. If we consider a value for $\sigma_{s}=0.001$, which is a reasonably conservative value from an atmospheric context, $S$ has to be approximately 1.002 to activate most of the particles. This corresponds to the mean-dominated regime, which requires that $S-S c \ll \sigma_{S}$. Additionally, when $S$ has a subcritical value, turbulence aids the activation process. For example, in figure 2.6, the second lowest activated fraction $\left(F_{n} \approx 0.1\right)$, can be achieved even if $S$ is subsaturated. This will correspond to a possible sub-regime of the fluctuation-dominated regime, when $S$ is subsaturated.

\subsection{Implications for the Atmosphere and Summary}

In the traditional view of activation in the atmosphere, based on a concept of a parcel of air rising through the atmosphere, the number fraction of aerosol that become cloud droplets is calculated based on the particles' size and chemical composition and the peak supersaturation in the parcel. [27, 28]. In this view, the environmental saturation ratio has a single peak value, and if it is larger than the critical supersaturation of an aerosol particle, the aerosol activates. Therefore, the larger and more hygroscopic particles become cloud droplets and the smaller and less hygroscopic particles remain as interstitials. However, in a turbulent environment, this concept of a single supersaturation is not a complete 


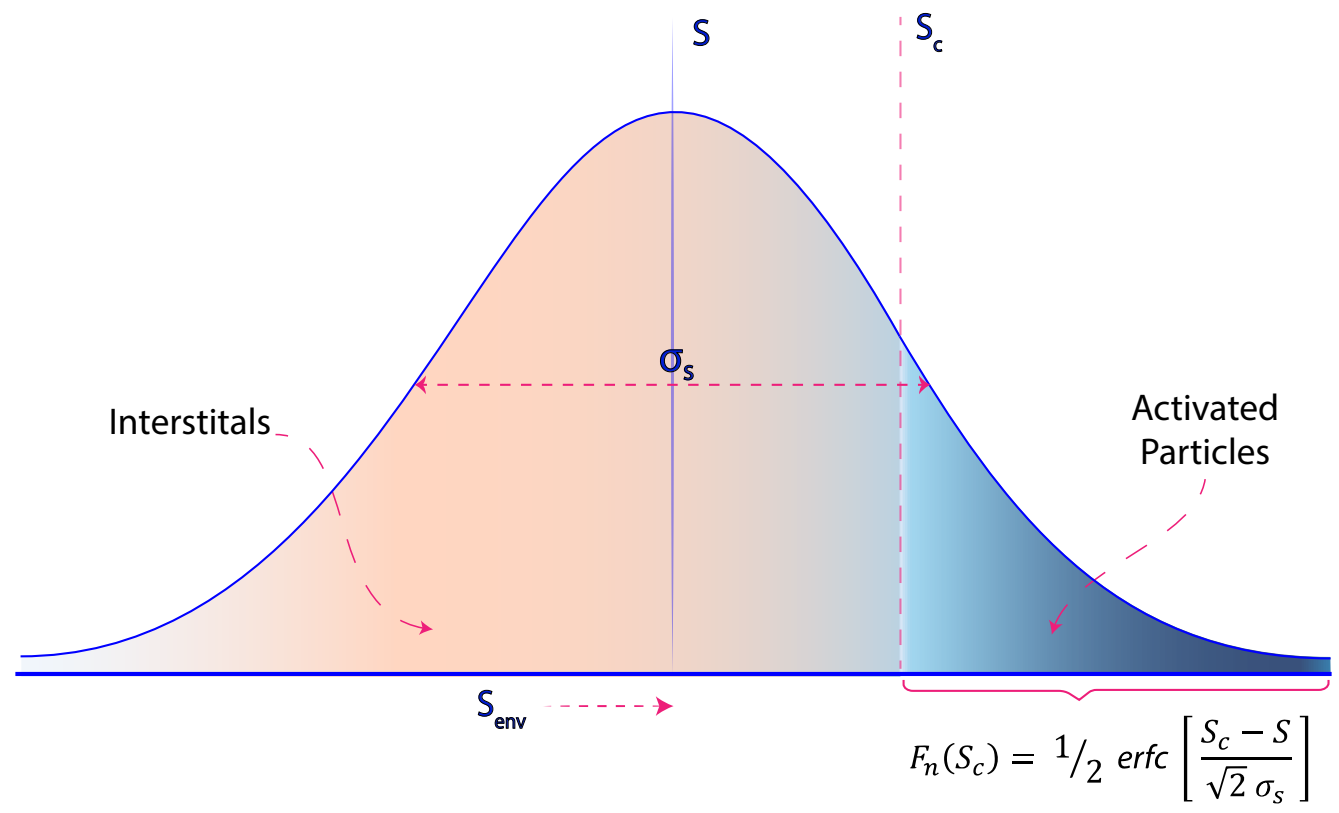

Figure 2.12: Schematic of the proposed model of $F_{n}$. The saturation ratio of the environment, $S_{\text {env }}$ is considered to have a Gaussian profile, with a mean $S$ (blue vertical line), and standard deviation, $\sigma_{S}$ (pink horizontal line). The red, dashed vertical line depicts the critical saturation of monodisperse and chemically homogeneous aerosol. The probability of a particle to be exposed to a saturation ratio larger than $S_{c}$ is represented by the shaded blue region. Eqn. 2.6 gives the area under the curve of that region.

picture of the aerosol activation process [63]. The saturation ratio in a turbulent environment is a distribution, rather than a single value, and hence, aerosols in a parcel have the possibility to be exposed to different "peak" saturation ratios. Viewed from that perspective, the dynamics of the activation/deactivation process are quite different.

These two views of supersaturation and activation can be further illustrated through a contrasting comparison between the regimes described by [2] (see also [55] and [64]) and those introduced by [3] and explored more completely here. In Sec. 2.6.2, these two perspectives were introduced in the context of our experiments. Here, we discuss them from a more holistic point of view. (See Fig. 
2.13 for a schematic representation.) [2] considered a parcel model to describe three distinctive regimes of activation. In this model, the source of supersaturation is an adiabatic cooling due to an updraft. We focus on the two extremes, the aerosol-limited and the updraft-limited regimes. The number of cloud droplets in the aerosol-limited regime is limited by the available CCN present in the system. The updraft, or forcing, is large enough to activate essentially all the CCN available. In contrast, the forcing in the updraft-limited regime is such that the peak supersaturation is low, and therefore, only particles with small $S_{c}$ (i.e., either relatively large or more hygroscopic particles) activate in this regime. These two regimes can be compared respectively with the mean dominated and the
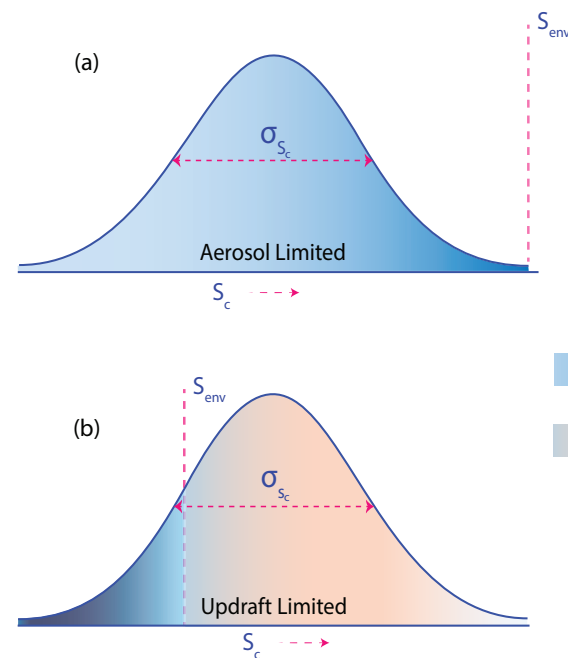
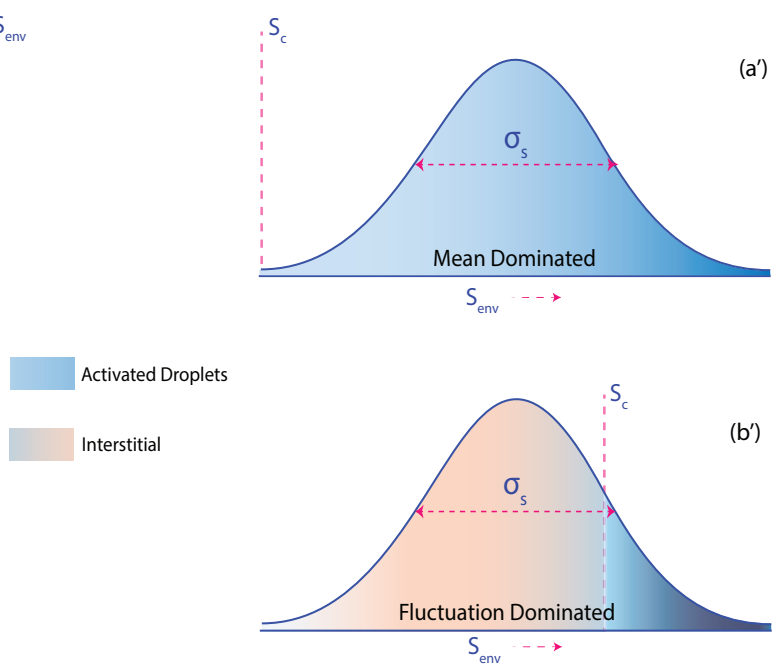

Figure 2.13: A schematic comparing the aerosol-limited (panel a) and updraftlimited (panel b) regimes described by [2], and the mean (panel a') and fluctuation dominated (panel $b^{\prime}$ ) regimes discussed here and by [3]. Note that the abscissas for panels a and $b$ are the aerosol critical saturation ratio, $S_{c}$, while in a' and $\mathrm{b}^{\prime}$, the abscissas are the saturation ratio in the environment, $S_{\text {env }}$. The variable considered changes because [2] considered polydisperse particles exposed to a single peak supersaturation in a parcel (vertical lines in the figure) while in [3] and here we have examined particles of a single size and composition (vertical lines in the figure) which are exposed to a distribution of saturation ratios in the environment. 
fluctuation dominated regime. In the mean dominated regime, the forcing, or mean supersaturation, $S_{0}$, is high enough to activate all the particles (e.g. the cleanest condition shown in Fig. 2.10). In the fluctuation dominated regime, the mean forcing is smaller than the required critical supersaturation to activate any particles. Particles in this regime activate only due to the turbulence-induced fluctuations in the saturation ratio (e.g. Fig. 2.8).

As Fig. 2.13 shows, an activated fraction of approximately 1 is possible from either of the viewpoints represented. If the forcing is large enough, all aerosol particles will act as $\mathrm{CCN}$, even in the presence of fluctuations in the saturation ratio. The figure also shows that a small activated fraction is possible in either case. The most striking difference is the possibility of activation even when the mean saturation ratio is equal to or less than unity. This phenomenon is only possible if the effects of fluctuations are considered.

In the atmosphere, these concepts may play a particularly important role in the transition from continental to maritime clouds (maritimization) or in the transition from stratocumulus to cumulus clouds. Clouds being swept from relatively particle-rich environments (e.g. continents) over cleaner ones (e.g. oceans) undergo a slow cleansing process that eventually results in a qualitative transition from a closed to an open cell convection pattern [65, 66]. This cleansing process is strongly influenced by predator-prey type interactions between aerosols, cloud droplets, and drizzle [14]. As noted in the Introduction, the dominant removal mechanism for the accumulation mode is precipitation, and even for modest drizzle rates, aerosol populations can be strongly modified by collection 
[67, 68, 69]. [70] have pointed out that in the absence of drizzle (or for imperceptible drizzle rates), clouds can act as a net loss for CCN through droplet coalescence. As we show above, fluctuations associated with turbulence can activate $\mathrm{CCN}$ that would remain as interstitial without it. In the initial stages of cleansing, when cloud droplet concentrations are high and in-cloud supersaturation low, activation because of fluctuations in the saturation ratio, with a subsequent reduction in the concentration of $\mathrm{CCN}$ in the cloud (through coalescence or low drizzle rates) may play a role in the cleansing that eventually leads to a qualitative transition [13, 69].

In summary, we performed a set of controlled experiments in Michigan Tech's $\Pi$ chamber to understand the effect of the mean saturation ratio, and fluctuations about the mean, on aerosol activation processes. Overall, we see that knowledge of a particle's size, chemical composition, and the mean supersaturation in the environment is not sufficient to quantify aerosol activation because of spatial and temporal variations in the saturation ratio induced through turbulence. In addition, we show relationships among the mean supersaturation, fluctuations in the supersaturation, and the activated fraction of aerosol. Finally, we showed that, for constant forcing, increasing aerosol concentration does result in an increase in cloud droplet number, but results in a smaller fraction of the aerosol being activated. 


\subsection{Acknowledgments}

This work was funded through the US Department of Energy, Office of Science grant DE-SC0018931 and by the National Science Foundation grant AGS1754244. The data presented here is available on Michigan Tech's Digital Commons, digitalcommons.mtu.edu . 
Dry Scavenging of Aerosol: Laboratory

Investigation of the Effect of Turbulence 


\subsection{Introduction}

Based on the contribution of precipitation (or activation), the removal processes of atmospheric aerosol can be categorized as either wet deposition or dry deposition. In the absence of precipitation, atmospheric particles can be removed by different dry removal processes [47, 71]. These processes include diffusion, impaction, interception, and gravitational settling.

Though wet deposition of some aerosol is estimated to be even four times larger than the corresponding dry deposition, the uncertainty associated with dry removal is substantial [72, 73]. Dry deposition is also an essential parameter for understanding indoor aerosol particles' transmission and their impact on human health [74]. This knowledge becomes more relevant considering the recent events of public health emergencies (e.g., the spread of COVID-19). Besides, it has also been argued that spatial distribution of aerosol deposition on lungs, along with its physiochemical properties, affects the toxicity of the particles [75].

The effect of turbulence on the dry deposition of aerosol was previously investigated through atmospheric measurements, targeted experiments, and modeling $[20,76,77,78]$. In the atmosphere, measuring dry removal requires the measurement of relative aerosol flux. Measuring aerosol flux comes with its difficulties and significant uncertainty [6, 72]. Knowledge of several other parameters such as vegetation, roughness, etc., is also necessary for these measurements.

Targeted experiments without turbulence focus only on specific size ranges. Experiments that considered turbulence generally use mechanical mixing, such as 
using a wind tunnel [76, 77]. Regardless of the consideration of turbulence, most of these experiments were carried out in chamber or vessel settings. Unlike the atmosphere, these chambers have sidewalls, and losses to the walls also need to be considered.

Loss mechanisms in a chamber can be characterized in tranquil and stirred settling conditions. In the absence of any fluid motion (i.e., tranquil condition), larger particles will settle solely due to gravity. In this environment, the number concentration of decaying monodisperse particles will follow a linear relationship with time. However, suppose there is a continuous and vigorous stirring mechanism present in the chamber so that the concentration of aerosol is always uniform throughout the chamber. In that case, both number concentration and the rate of the change in the number concentration change with time. Therefore, this relationship could be given by -

$$
\begin{aligned}
& \frac{d N_{a, t}(t)}{d t} \propto N_{a, t} \\
& \frac{d N_{a, t}(t)}{d t}=-\beta N_{a, t}
\end{aligned}
$$

where, $N_{a, t}(t)$ is the number concentration at a time, $t . \beta$ is known as the deposition coefficient. Earlier literature suggested a simple form of $\beta$, considering the loss due to diffusion and gravitational settling [76, 79].

$$
\beta=\frac{D_{v} S}{\delta_{\mathrm{dbl}} V}+\frac{v_{s t}}{H}
$$


Here, $S, V$, and $H$ denotes the surface area, volume, and height of the chamber respectively. $\delta_{\mathrm{dbl}}$ is the width of the diffusive boundary layer. $D_{v}$ and $v_{s t}$ are the diffusivity and the settling velocity of the particles, defined as [80]

$$
\begin{aligned}
& v_{s t}=\frac{\rho D_{p g}^{2} g C_{c}}{18 \eta} \text { for } R e \leq 1 \\
& D_{v}=\frac{k T C_{c}}{3 \pi \eta D_{p}}
\end{aligned}
$$

here, $R e$ is the Reynolds number of the particle, $D_{p}$ is the diameter of the particle, $k$ is the Boltzmann constant, $T$ is the absolute temperature, $\eta$ is the gas viscosity, $\rho$ is the particle bulk density, $g$ is the gravitational acceleration, and $C_{c}$ is the Cunningham slip correction factor.

Along with mixing, equation 3.2 assumes that Brownian diffusion is the only diffusive transport mechanism and is appreciable only near the wall. Therefore, gravitational settling (the second term of equation 3.2) is independent of diffusive settling (the first term).

Crump et al. [76] argued that the form of $\beta$ given by equation 3.2 is inadequate for general cases, and the diffusive term is not entirely independent of the gravitational settling . However, in this dissertation, we discuss some preliminary results, and exploring the complexity of $\beta$ is beyond this report's scope.

Our motivation behind this work is to understand and quantify the turbulenceinduced dry removal rate and the size dependency of the effect of turbulence. It would also help us quantify the residence time of aerosol particles in the chamber as a function of $\Delta T$, without additional challenges posed by the humidity. 
We discuss the method used for calculating a decay time constant in the method section. Following that, the results are discussed. In the summary section, we discuss the caveats of our experiments and propose some experiments as possible future work.

\subsection{Method and Instrumentation}

We performed targeted sets of experiments to explore the effect of turbulence on the dry removal of aerosol and any corresponding size or composition dependency of the aerosol. The experiments were carried out in Michigan's Tech Pi chamber, which is a Rayleigh-Bénard convection chamber. A short description of this chamber was discussed in section 2.5, and further technical details can be found in Chang et. al. [36].

The turbulence intensity of this chamber can be controlled by creating a temperature gradient between the top and bottom plates. Unlike the cloudy conditions discussed in section 2.4 the relative humidity for these experiments was maintained below the aerosol particles' deliquescence relative humidity (DRH). This condition ensures dehydrated aerosol particles (i.e., no condensation on aerosol particles). The Rayleigh number of the flow in this condition can be described using the first part of equation 2.4, and the turbulent kinetic energy dissipation rate can be determined by equation 2.5 using the new Rayleigh number.

In all cases, mobility size-selected aerosol particles were used, which helped reduce the uncertainty of polydisperse particles [77]. The aerosol composition was 
changed either to obtain a larger diameter or a different density. Note that, between diffusion and Stokes settling, only the latter has a density dependence. In contrast, deposition due to diffusion is independent of the density and depends only on the size of the particles.

Size selection of the aerosol particles was obtained using either DMA (TSI model 3071) or an aerodynamic aerosol classifier (AAC, Cambustion). DMA is based on the electric mobility diameter of a particle, while AAC selects the sizes based on the equivalent aerodynamic diameter. However, the focus is on the relative comparisons, and conversion between aerodynamic diameter to mobility diameter (and vice versa) is possible for a known shape factor.

After size selection, aerosol particles were injected into the chamber. The injection was stopped after reaching a substantial number concentration. Since a temperature gradient was always maintained for each experiment, and the flow is fully turbulent, it is safe to assume that the aerosol was well mixed and that the concentration was uniform throughout the chamber. Therefore, following the relation given in equation 3.1a, the decay in number concentration is

$$
N_{a, t}(t)=N_{a, 0} \exp \left[\frac{-t}{\tau_{\text {decay }}}\right]
$$

Here, $N_{a, 0}$ is the initial number concentration (i.e. at $t=0$ ), and $\tau_{\text {decay }}$ is the decay time constant. $\tau_{\text {decay }}$ is defined as the time that requires for the number concentration to reach $1 / e$ of the original number concentration. In our case, $\tau_{\text {decay }}=1 / \beta$. Since, $\beta \sim 1 / D_{v}$ (from equation 3.2), $\tau_{\text {decay }} \sim D_{v}$. 
Taking a natural logarithm on the both side of equation 3.4, gives us equation of a straight line, where $-\frac{1}{\tau_{\text {decay }}}$ is the slope. Both $N_{a, 0}$ and $N_{a, t}(t)$ were measured using the SMPS system. Figure 3.1 shows one of the examples to calculate $\tau_{\text {decay }}$.

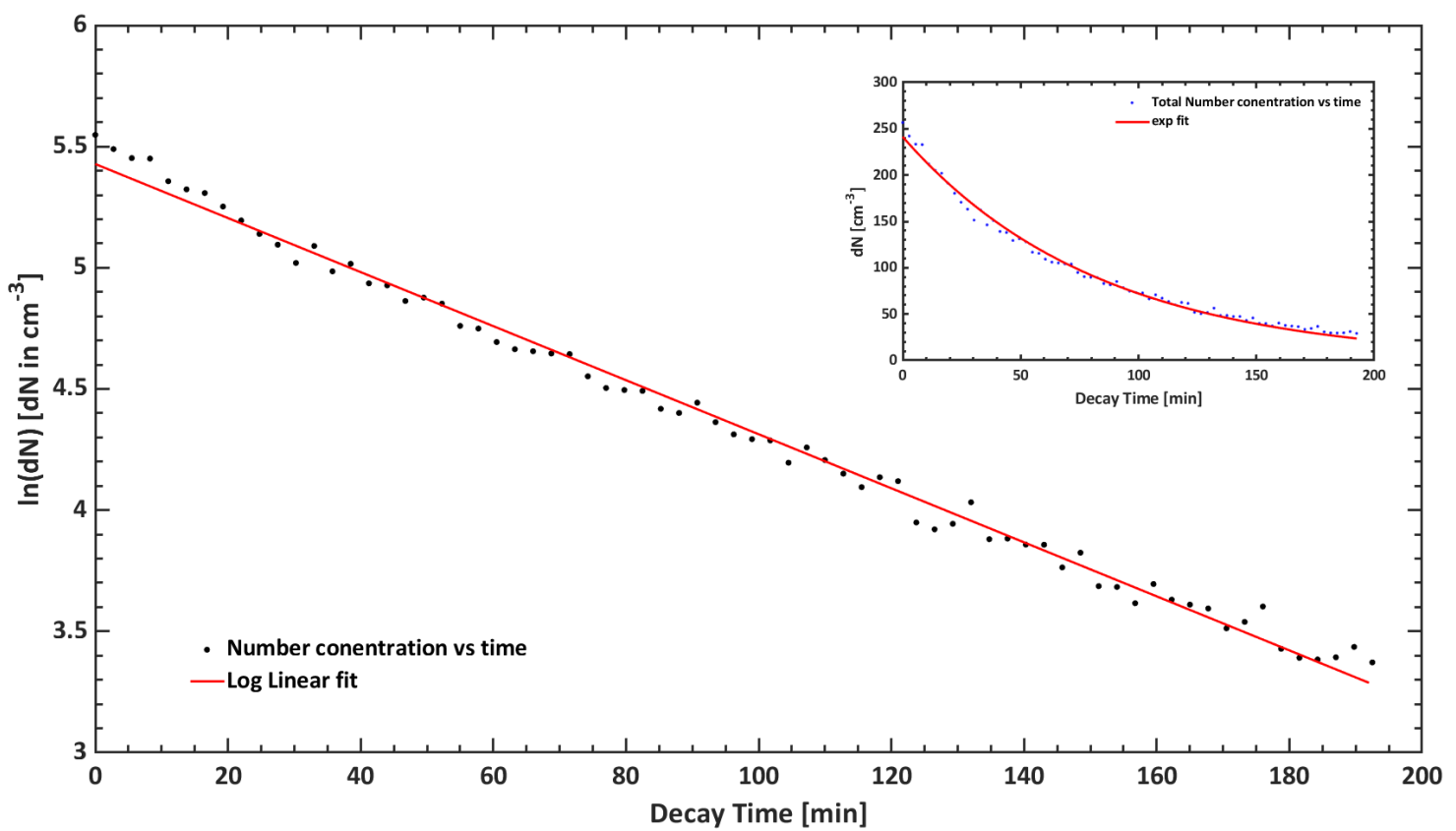

Figure 3.1: An example of calculating $\tau_{\text {decay }}$ from linear fit from natural log of the measured number concentration. Only the $70 \mathrm{~nm}$ size bin was considered in this particular case. $\tau_{\text {decay,70 }}$ calculated from the fit was 90 minutes. The corresponding number concentration data and the exponential fit of the data are shown in the inset. The same method was applied for calculating $\tau_{\text {decay }}$ in other experiments as well.

\subsection{Result and Discussion}

\subsubsection{Effect of Turbulence on $\tau_{\text {decay }}$}

In our attempt to understand how turbulence may affect the dry deposition of aerosol, we performed experiments where we varied the turbulent intensity and size of the aerosol. The primary motivation for the series of experiments is to 
understand if turbulence affects the dry removal of aerosol. For this set of experiments, we used size-selected $\mathrm{NaCl}$ and $\mathrm{AgI}$ as our compositions. The diameters considered in these cases were $70 \mathrm{~nm}, 130 \mathrm{~nm}, 200 \mathrm{~nm}$, and $300 \mathrm{~nm}$. The size selections were performed through a DMA.

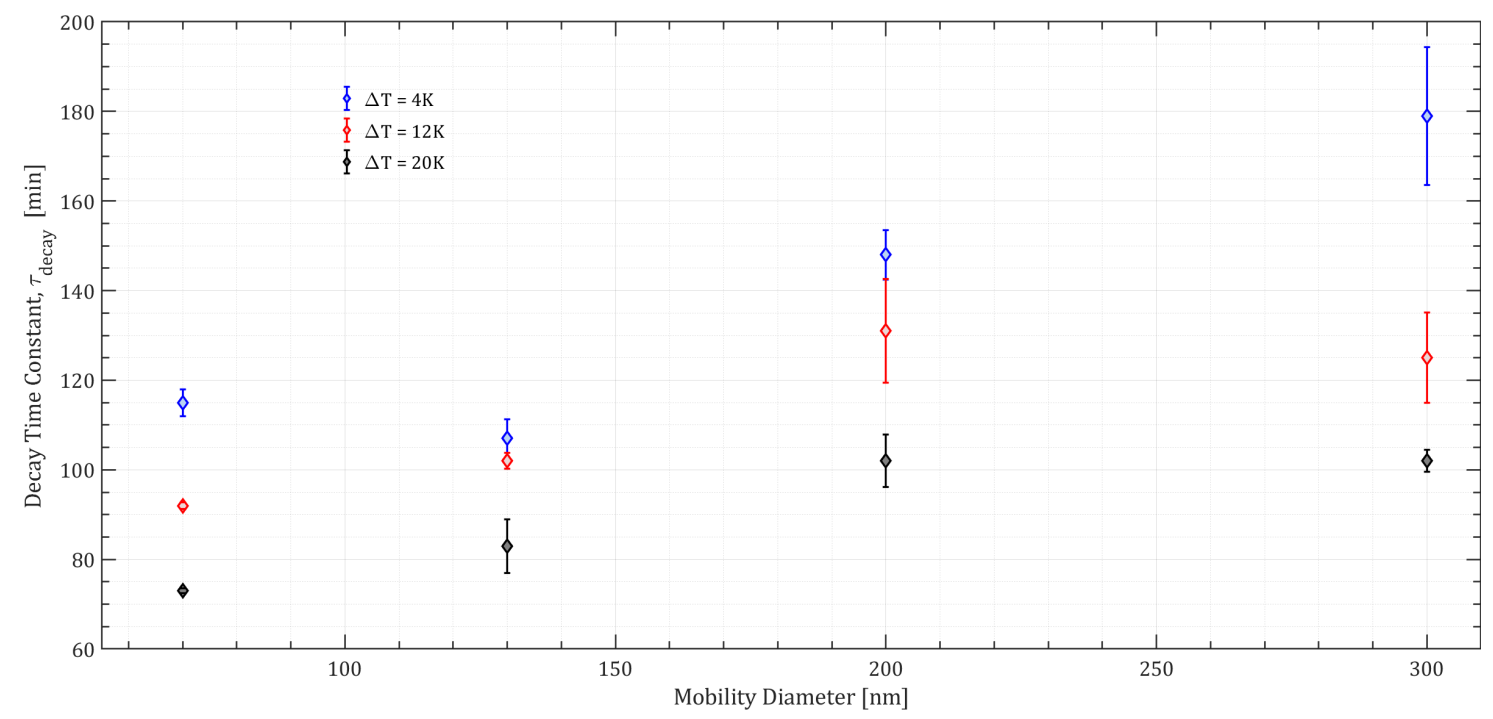

Figure 3.2: $\tau_{\text {decay }}$ as a function of $D_{p}$ at different $\Delta T$. Blue, red and black points correspond to $4 \mathrm{~K}, 12 \mathrm{~K}$, and $20 \mathrm{~K}$, respectively. $\tau_{\text {decay }}$ were calculated considering five neighboring bins of the selected sizes. The markers show the mean $\tau_{\text {decay }}$ of those five bins, and the uncertainty bars correspond to 1 standard deviation.

The reason to choose two different chemical compositions in these cases were, 1) not being able to have a significant number concentration from large $\mathrm{NaCl}$ particles, 2) because of the propensity for coagulation, it was difficult to produce small AgI particles. Note that, $\mathrm{NaCl}$ and $\mathrm{AgI}$ have different density of $2.17 \mathrm{~g} / \mathrm{cm}^{3}$ and $5.68 \mathrm{~g} / \mathrm{cm}^{3}$ respectively. According to earlier literature, dry deposition of this size range is primarily propelled by the diffusion process [20, 78, 81]. Since the diffusivity depends only on the size of the particles, we assumed that the effect of density would be negligible in this size range. 
The three $\Delta T$ used in these cases were $4 \mathrm{~K}, 12 \mathrm{~K}$, and $20 \mathrm{~K}$. Since $D_{v} \sim$ $1 / D_{p}$ and $\tau_{\text {decay }} \sim 1 / D_{v}$, we examined the changes in $\tau_{\text {decay }}$ as a function of $D_{p}$. Three colors blue, red, and black represent $\Delta T=4 \mathrm{~K}, 12 \mathrm{~K}$ and $20 \mathrm{~K}$ respectively. This figure shows that turbulence is aiding the dry loss mechanism at this size range. Also, the linear relation between $\tau_{\text {decay }}$ suggests that diffusion is the dominant mechanism, considering equations 3.2 and $3.3 \mathrm{~b}$.

Another parameter that may affect the diffusive settling is the thickness of the diffusive boundary layer, $\delta_{d b l} . \delta_{d b l}$ was calculated using equation 3.2 . Harrison et. al. argued that molecular diffusion is significantly predominant inside $\delta_{d b l}$, and $\delta_{d b l}$ is a function of $D_{p}$ [82]. Figure 3.3(a) and 3.3(b) shows $\delta_{d b l}$ as a function of $D_{p}$ and as a function of $\Delta T$, respectively. Figure 3.3 (a) exhibits a dependence of $\delta_{d b l}$ on $D_{p}$. Though quantitatively different, this dependence of $\delta_{d b l}$ on $D_{p}$ was previously reported by Harrison et al. [82]. However, these results are
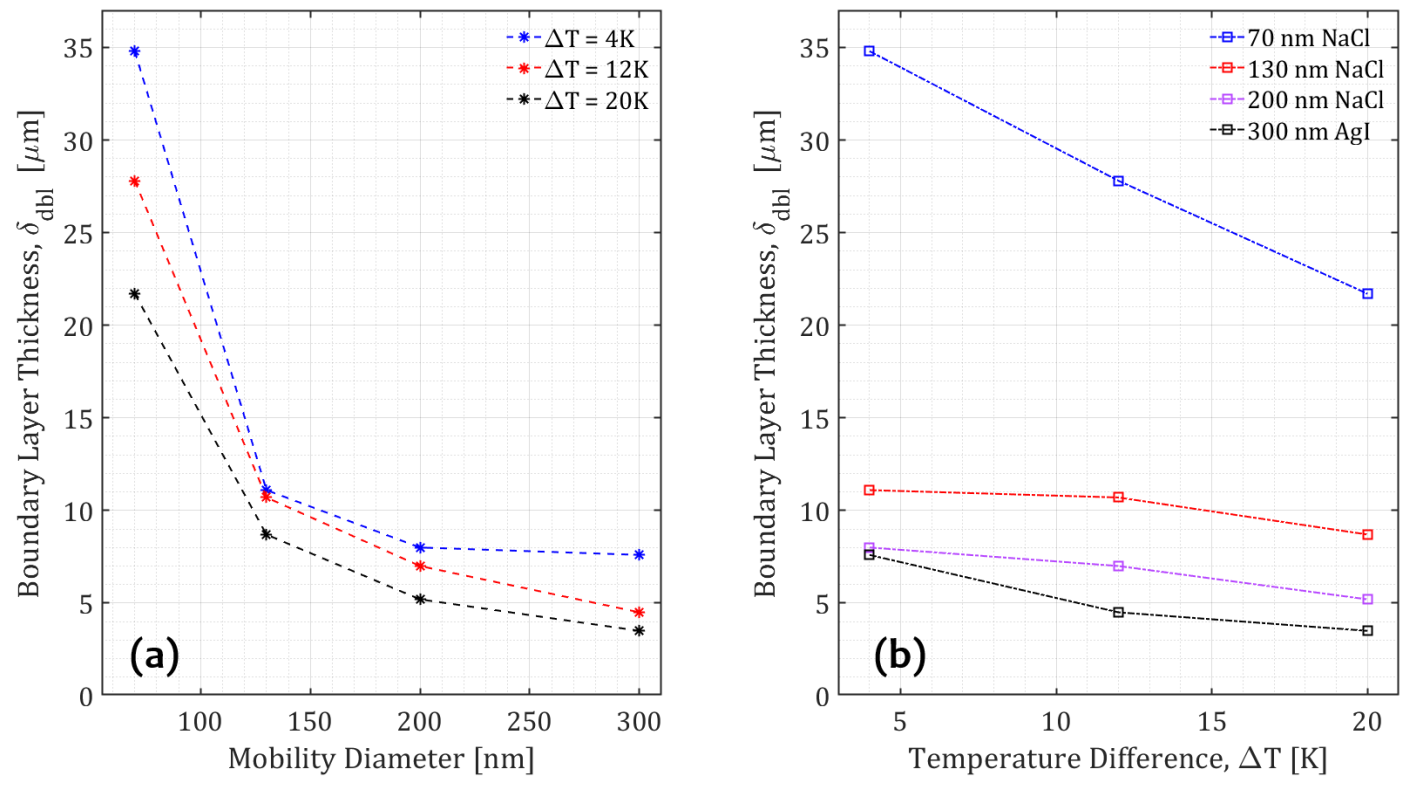

Figure 3.3: (a) $\delta_{d b l}$ as a function of the mobility diameter, $D_{p}$ at different $\Delta T$. (b) $\delta_{d b l}$ as a function of $\Delta T$ for different sizes of the particles. 
qualitatively similar. We also found a decrease in the boundary layer thickness with increasing particle diameter, as Harrison et al. suggested. The quantitative difference between this work and Harrison et al. can come from differences in aerosol composition, shape factor, container shape, and turbulent intensity, among other factors. Figure 3.3(b) suggests that $\delta_{d b l}$ decreases with increasing $\Delta T$ (i.e., increasing turbulent intensity).

\subsubsection{Other Preliminary Data}

In the previous section, we discussed the impact of turbulence intensity on $\tau_{\text {decay }}$. In this section, we will discuss some preliminary data to understand if this effect of turbulence has any size dependency.

To understand the effect of aerosol, we conducted a set experiments where $\Delta T$ was kept same but we changed the size of the aerosol particles. We used sizeselected $\mathrm{NaCl}$ and Arizona Test Dust (ATD). $\mathrm{NaCl}$ and ATD have a similar density $\left(2.17 \mathrm{~g} / \mathrm{cm}^{3}\right.$ and $2.65 \mathrm{~g} / \mathrm{cm}^{3}$ respectively). Since the Stokes settling velocity $v_{S t}$ of a particle depends more on diameter $\left(\sim D_{p}^{2}\right)$ than its density $(\sim \rho)$, the change in density of these two particle would not significantly change $v_{S t}($ e.g., $800 \mathrm{~nm} \mathrm{NaCl}$ and ATD have $v_{S t}$ of $51 \mu \mathrm{m} / \mathrm{s}$, and $61 \mu \mathrm{m} / \mathrm{s}$ respectively).

We used different instrumentation to select the mobility size of the particles. As discussed earlier in section 3.2, though AAC measures the aerodynamic diameter of the particles, the corresponding mobility diameter can be calculated if the shape factor is known. The mobility diameters chosen for these experiments were $55 \mathrm{~nm}, 200 \mathrm{~nm}, 800 \mathrm{~nm}$ for $\mathrm{NaCl}$ and $1.6 \mu \mathrm{m}$ and $3.2 \mu \mathrm{m}$ for ATD. These 
Table 3.1: A table showing some preliminary data from the experiments conducted to understand the effect of size on dry removal of aerosol in a turbulent environment. The column Instrument Used correspond to the instrument that was used to size-select the aerosol and measuring the size distribution afterwards. $\mathrm{AAC}=$ Aerodynamic Aerosol Sizer, DMA $=$ Differential Mobility Analyzer, $\mathrm{CPC}=$ Condensation Particle Counter, and SMPS = Scanning Mobility Particle Sizer.

\begin{tabular}{|c|c|c|c|c|}
\hline Mobility Diameter & $\Delta \mathrm{T}$ & Instrument Used & Composition & $\tau_{\text {decay }}$ \\
\hline $\mathbf{n m}$ & $\mathbf{K}$ & & & $\min$ \\
\hline \multirow{2}{*}{50} & \multirow{2}{*}{19} & DMA + SMPS & \multirow{2}{*}{$\mathrm{NaCl}$} & 66 \\
\hline & & $A A C+S M P S$ & & 56 \\
\hline \multirow{5}{*}{200} & \multirow{5}{*}{19} & $\mathrm{DMA}+\mathrm{CPC}$ & \multirow{5}{*}{$\mathrm{NaCl}$} & 102 \\
\hline & & DMA + SMPS & & 96 \\
\hline & & DMA + SMPS & & 98 \\
\hline & & $\mathrm{DMA}+\mathrm{SMPS}$ & & 96 \\
\hline & & $A A C+$ SMPS & & 101 \\
\hline \multirow{2}{*}{800} & \multirow{2}{*}{19} & $\mathrm{AAC}+\mathrm{CPC}$ & $\mathrm{NaCl}$ & 166 \\
\hline & & $A A C+C P C$ & ATD & 121 \\
\hline 1600 & 19 & $\mathrm{AAC}+\mathrm{CPC}$ & ATD & 121 \\
\hline 3200 & 19 & $A A C+C P C$ & ATD & 128 \\
\hline
\end{tabular}

sizes were carefully selected because of their preference for different removal mechanisms. Diffusion is the prevalent removal mechanism for $55 \mathrm{~nm}, 200 \mathrm{~nm}$, while gravitational settling is the primary mechanism for $3.2 \mu \mathrm{m}$ aerosol. $800 \mathrm{~nm}$ and 1.6 $\mu \mathrm{m}$ are in the transitional regime where both diffusion and gravitational settling play a significant role [20, 78].

Note that the data presented in table 3.1 is preliminary. The difference in the shape factors between $\mathrm{NaCl}$ and ATD imposes a large enough uncertainty that any conclusions remain ambiguous. It requires a more targeted experiment to eliminate these uncertainties. In the summary section (section 3.4), some possible experiments are suggested. 


\subsection{Summary}

Two sets of targeted experiments were performed to understand the effect of turbulence on the dry removal of aerosol particles of different sizes. We showed that for the same sizes, increasing turbulent intensity aids the dry removal of aerosol. We also showed that for a given turbulent intensity, the removal process has size dependency.

While these experiments provided valuable information, some significant uncertainties could not be evaluated. For example, for the experiment described in section 3.3.1. larger diameter aerosol could not be used because of the unavailability of the proper instrumentation. The experiments with larger size particles needed to be performed to explore if the same trends hold for the larger diameters. Besides, we have used two different chemical compositions in both sets of experiments described in section 3.3.1 and section 3.3.2. In the first case, $\mathrm{AgI}$ has approximately three times larger density than $\mathrm{NaCl}$. This difference in density may have a significant effect on the larger sizes and may introduce ambiguity in the results. Secondly, though $\mathrm{NaCl}$ and ATD have similar densities, they have pretty different shape factors. For example, $\mathrm{NaCl}$ has a shape factor of 1.08 while the shape factor of ATD varies (ranging 1.05 - 1.88) [80]. Therefore, we propose experiments using the same chemical composition with known properties such as density, dynamic shape factor, etc. We also propose to vary the turbulent intensity (from isothermal condition to highest achievable $\Delta T$ in a dry condition). Figure 3.4 shows the proposed experiments in a nutshell. 
$\sim$ Use Same Aerosol throughout

e.g., Polystyrene Latex (PSL) - same shape factor + same density

$\sim$ Scan through the following sizes $\rangle \gg\rangle$
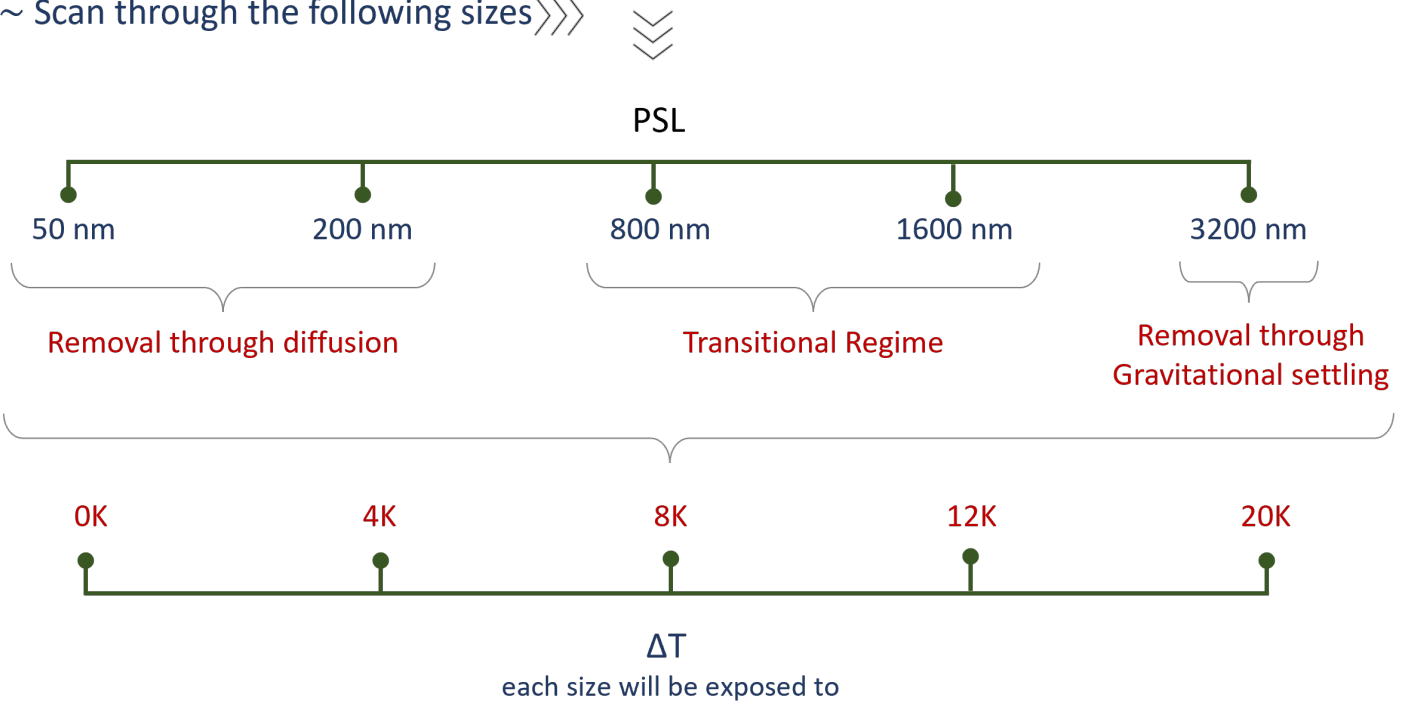

Figure 3.4: Figure showing the future experiments that can be performed to determine the effects of turbulence and quantify a residence time of aerosol as a function of size and turbulent intensity 



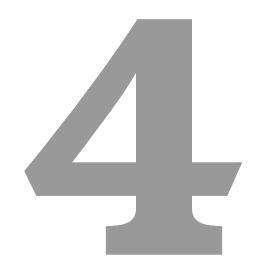

\section{To Improve the Temporal Resolution of a}

\section{Dual Column CCN Counter}




\subsection{Introduction}

How readily an aerosol particle acts as a seed for cloud droplets, has important consequences on the Earth's radiative budget [83]. Given the governing parameters for aerosol activation such as ambient supersaturation, size, and chemical composition of aerosol, the Köhler equation and its consequent parameterization (such $\kappa$-Köhler equation) can predict the probability of aerosol-activation [8, 44, 45]. However, the physio-chemical properties of atmospheric aerosol are too complex and too variable to predict the $\mathrm{CCN}$ concentration in the field and laboratory settings using theories, and requires reliable measurement capabilities [84, 85].

Historically, different kinds of CCN counters have been around and widely used since Twomey(1963) [86]. The type of CCN counters Twomey developed creates supersaturation by generating a temperature gradient between two parallel, wet horizontal plates. Though some of the limitations were resolved by better instrumentation and calibration, these thermal gradient diffusion chambers were always limited by the minimum achievable supersaturation and poor temporal resolution [87, 88]. For example, the lowest achievable set supersaturation in this type of setup is $\approx 0.2 \%$ which is more than the supersaturation found in some marine stratus clouds [89, 90]. The uncertainty associated with the CCN concentration measurements using Twomey counter is also high [90, 91].

A number of these limitations are overcome by designing $\mathrm{CCN}$ counters in cylindrical shapes that create supersaturations along the axial direction by creating stream-wise thermal gradients [89, 90, 92]. These $\mathrm{CCN}$ counters operate based on 
the difference in thermal diffusivity and water vapor diffusivity [89, 90, 92, 93]. The design from Roberts \& Nenes (2005) showed advantages such as obtaining constant supersaturation, higher resolution than predecessors, etc. It was later commercialized by Droplet Measurement Technology (DMT) [94].

The effective supersaturation of a DMT CCN counter (DMT-CCNC) depends on the thermal gradient, pressure, and the flow rate. Conventionally, supersaturation in a DMT-CCNC is changed by changing the temperature gradient along the streamline of aerosol while keeping the flow and pressure constant. Since temperature stabilization is a slow process, this step-wise change in thermal gradient translates to a slow change in supersaturation. For example, it may take over 3 mins to stabilize the instrument temperature and the optical particle counter temperature [95]. During airborne and field campaigns where the meteorological conditions can change quickly, these limitations may significantly affect the $\mathrm{CCN}$ activity measurements. We significantly improved this resolution by altering some built-in instrumentation and developing a new method of operation where flow parameters varied instead of the temperature gradient. We also obtained a calibration curve showing relative humidity as a function of the flow parameters (in contrast to the traditional relative humidity as a function of temperature gradient curve).

In the following section, we will discuss the instrumentation and method we followed. Then, we will present the calibration plot, the caveats in our assumptions and possible future improvements. Lastly in the summary section, we will discuss the benefits of using this methodology in an atmospheric field measurement context. 


\subsection{Method and Instrumentation}

A regular calibration of the instrument was performed before continuing with our methodology. A calibration curve was obtained relating $\Delta T$ and set supersaturation. Size-selected ammonium sulfate was used for performing the calibration. The workflow, example figure, and the calibration curve are discussed in appendix $\mathrm{D}$.

\subsubsection{Parameter Setup}

The methodology is inspired by an earlier work by Moore \& Nenes [95]. This mode of operation of DMT-CCNC, known as the scanning flow CCN analysis (SFCA), has been used in several field measurements and laboratory experiments [96, 97, 98]. In our case, we used a dual column DMT-CCNC. As the name suggests, a dual column DMT-CCNC has two identical cylinders (or columns) instead of one. It has the same working principle as a single column DMTCCNC. The control parameters of these two columns can be set independently, resulting in a wider range of achievable supersaturation. We have also automated the flow control as a possible use in field measurements. We realized that the following three questions need to be answered before starting the calibration in scanning mode:

1. Given a constant pressure, $P$, and a constant temperature difference, $\Delta T$ along the aerosol streamline, how are the flow rate, $Q_{T}$, and the supersaturation, $S_{c c n c}$ along the streamline related? 
2. Given the changes in $Q_{T}$, would particles have adequate time to grow enough to be detected by the optical particle counter (OPC)? And,

3. Given the answers for the last two questions, what would be an adequate range of $Q_{T}$, and how fast can it be scanned through, i.e., what would be an adequate ramp time, for a given $Q_{T}$ ?

Both Robert \& Nenes (2005), and Moore \& Nenes (2009), showed that for standard pressure, the supersaturation along the axial direction changes linearly with the flow [89, 95]. Moore \& Nenes (2009) carried out simulations that showed that even for the highest flow rate $\left(Q_{\max }\right)$ of $1 \mathrm{lpm}$, particles would grow large enough to be detected by the OPC. However, (a) those particles have to be

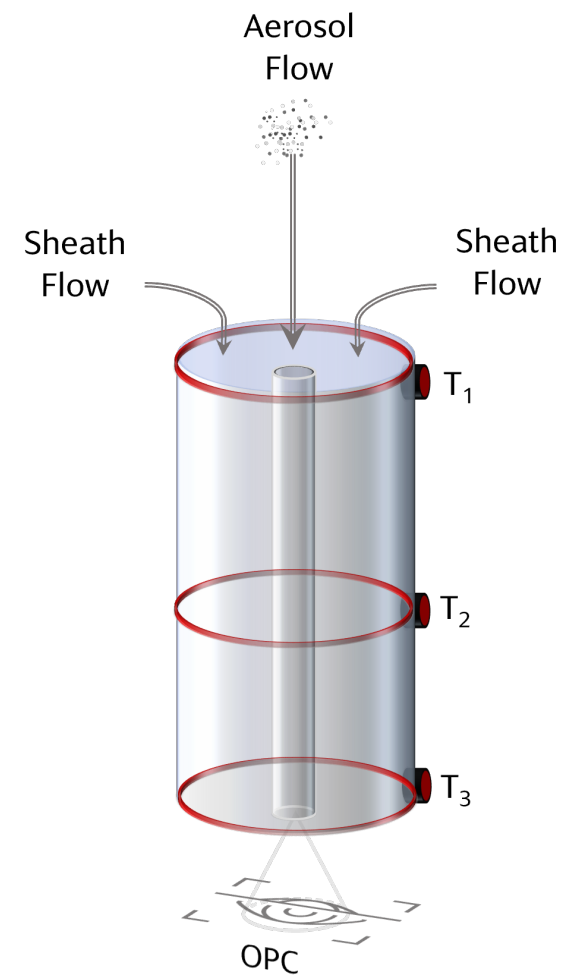

Figure 4.1: Basic Illustration of a column of a DMT-CCNC. For a dual column DMT-CCNC, there are two similar columns. Aerosol flow, sheath flow, $T_{1}, T_{2}, T_{3}$, and OPC temperatures of each column can be controlled separately. The inner cylinder is made of thermally conductive materials and kept wet. A thermal difference, $\Delta T$ along $T_{1}$ and $T_{3}$ creates a supersaturation along the streamline of the aerosol flow. The inner cylinder illustrates the aerosol streamline. 
hydrophilic, and (b) the flow rate has to correspond to the particles' supersaturation, $Q_{C}>Q_{\max }$. It was also shown that the relation between the supersaturation and the flow rate deviates from linearity for a ramp time of less than $30 \mathrm{~s}$.

Considering the findings in earlier literature, we set the pressure, $P_{c c n c}$ at $1 \mathrm{~atm}$, $\Delta T$ of $18 \mathrm{~K}$, and $6 \mathrm{~K}$, along column $\mathrm{A}$ and column $\mathrm{B}$, respectively. The range of flow rate we considered was between $0.2 \mathrm{lpm}$ and $1 \mathrm{lpm}$. We chose size-selected ammonium sulfate for this calibration, scanning through $25 \mathrm{~nm}$ to $160 \mathrm{~nm}$ with intervals.

\subsubsection{Instrumentation}

Figure 4.2 shows the instrumentation set that was used for the calibration process. A $0.003 \mathrm{wt} \%$ ammonium sulfate solution (using DI water as solvent) was used for the experiment. This solution was aerosolized using a constant output atomizer (TSI model 3076). These particles then went through a diffusion dryer and were size selected through a DMA (TSI model 3071). A condensation particle counter (CPC, TSI model 3750), measured the total number concentration of particles that were generated by the atomizer. Three mass flow controllers $\left(M_{m}, M_{a}, \& M_{b}\right)$ were used to control the flows. Green arrows in figure 4.2 show the directions of the flows. The DMA and CPC operate on a constant flow of 0.3 lpm. The internal pumps of the dual column DMT-CCNC were replaced with external pumps and the flows through column A and column B were controlled by $M_{a}$ and $M_{b} . M_{m}$ supplies the dry air required to make up the flow necessary to have a constant flow through the CPC. 
All the flow controllers were equipped and controlled with micro-controllers (Arduino Model Uno Rev3). The flow controlled by $M_{m}, M_{a}, \& M_{b}$ follows a cosine function given by -

$$
Q_{T}(t)=m_{m}+a_{m} \times \cos \frac{\pi \times t}{T}
$$

where, $t$ is time in seconds, $T$ is the time period $m_{m}$ is the mid-point of the distribution, and $a_{m}$ is the amplitude. 


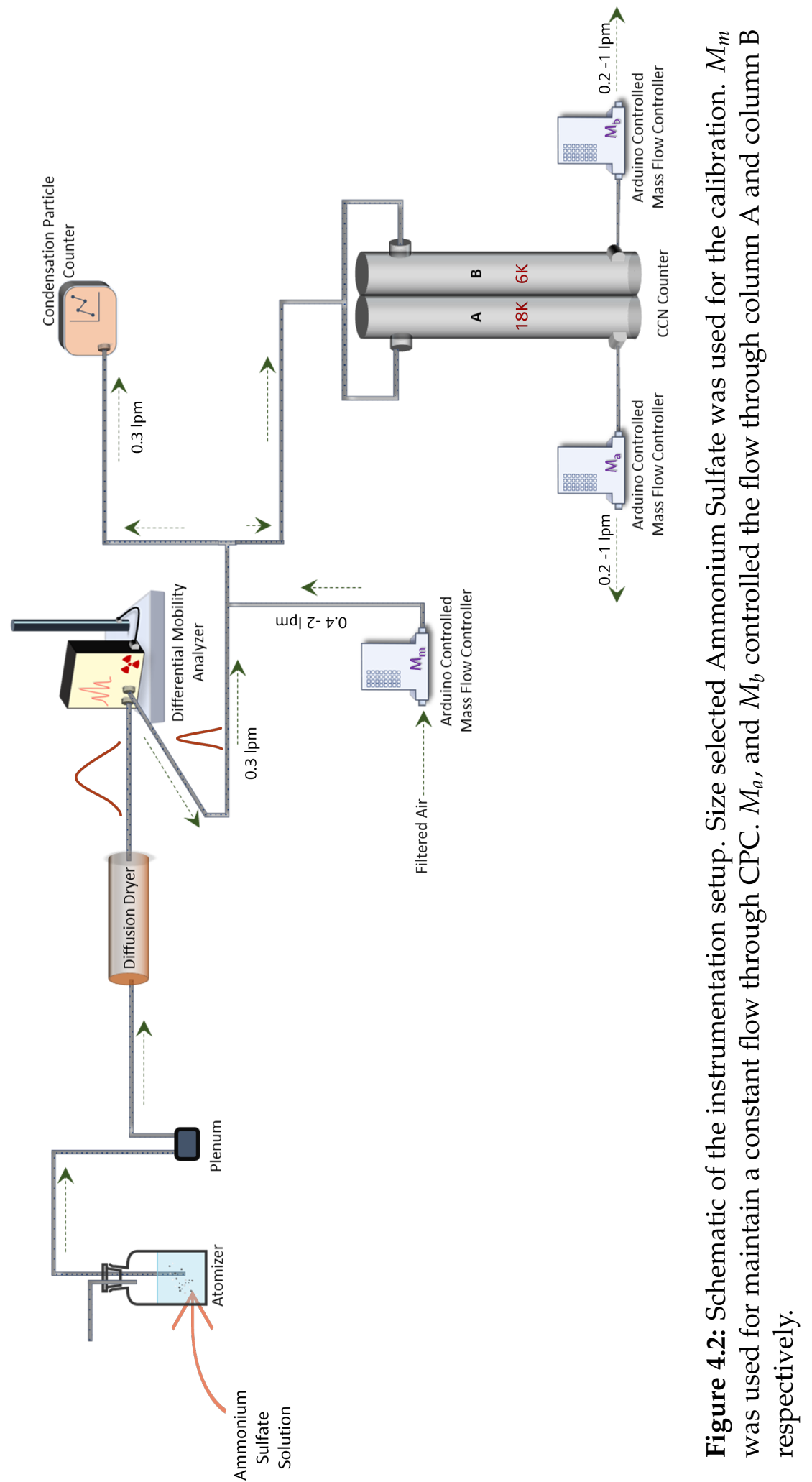




\subsubsection{Calibration method}

For the calibration process we scanned through $25 \mathrm{~nm}-160 \mathrm{~nm}$ diameter size selected ammonium sulfate. Ammonium sulfate has known hygroscopicity, and therefore, known critical supersaturation. The diameters used and their respective supersaturation are documented in a table in appendix $D$.

After size selecting the particles through a DMA (with a sheath flow of $3 \mathrm{lpm}$ and aerosol flow of $0.3 \mathrm{lpm}$ ), the number concentration was measured by both by a CPC and the dual columns DMT-CCNC. Constant thermal gradients on column A and column B (see figure 4.2) were set at, $\Delta T_{A}=18 \mathrm{~K}$, and $\Delta T_{B}=6 \mathrm{~K}$ respectively. $Q_{T}$ for both column A and column B changed between $0.2 \mathrm{lpm}$ and $1.0 \mathrm{lpm}$ using eqn 4.1. $T$ was set to be 60 seconds. To maintain a constant flow through the CPC, dry air was passed through $M_{m}$ following the same equation. Every cycle contained one 60s lowering flow (down-scan), 30s constant flow and one 60s rising flow (up-scan). Figure 4.3 exemplifies one of cycles of $Q_{T}$ through column A and column B.

Even though the CPC and DMT-CCNC were closer to each other, there was a time lag between them. We measured the maximum and minimum time lag (which corresponds to the minimum and maximum $Q_{T}$ respectively). Using those maximum and minimum value, we calculated the time lag for each $Q_{T}$ following eqn 4.1 . This time lag was taken under consideration while calculating the activated fraction. In this case, the activated fraction is defined as -

$$
F_{n, C C N}=\frac{\text { Number Concentration of Cloud Droplets, } N_{C C N}}{\text { Total Number Concentration of aerosols, } N_{C P C}}
$$



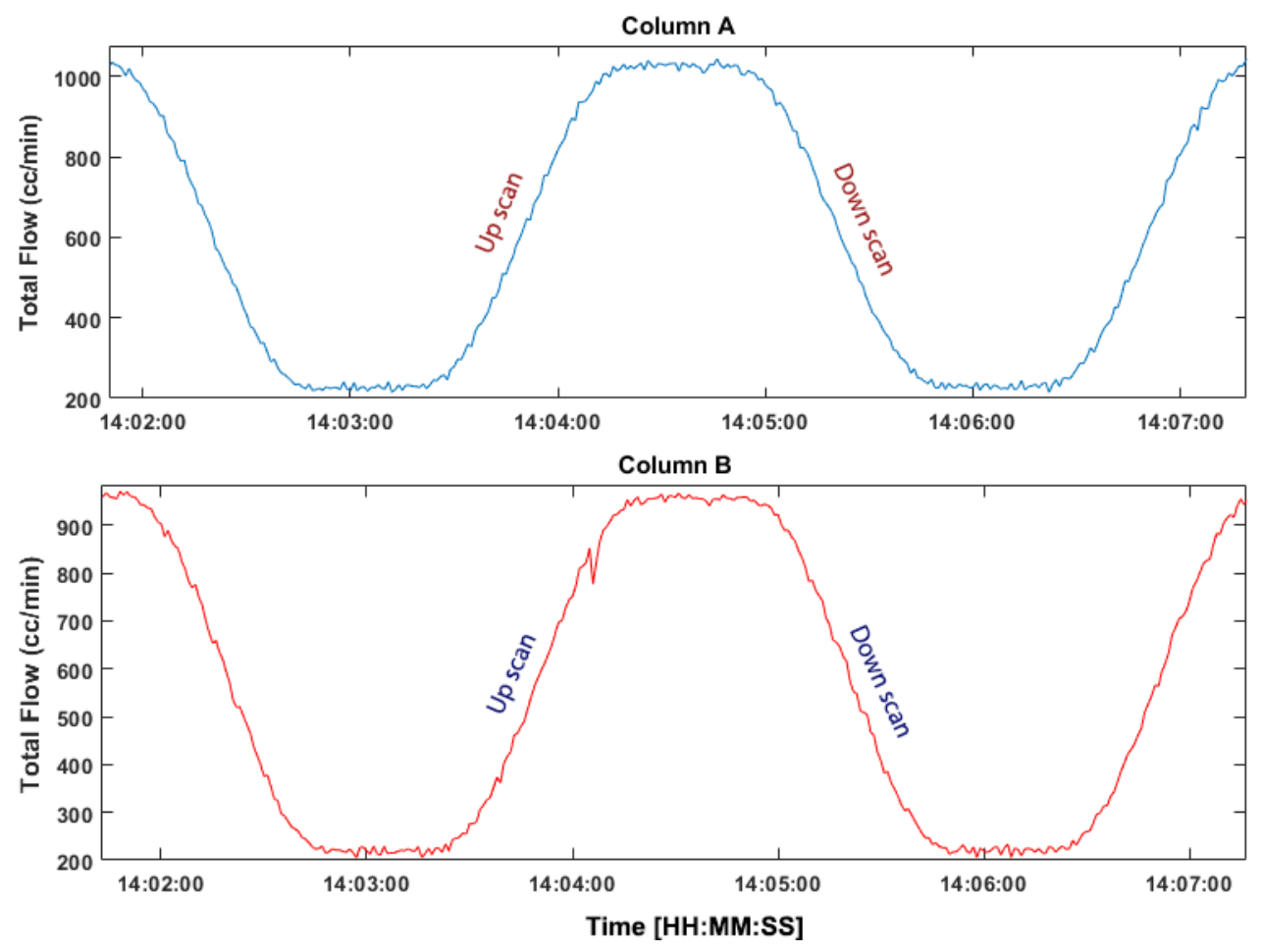

Figure 4.3: Example shows one of the flow cycles following eqn 4.1. Note the rising and lowering part of the flow denoted as up scan and down scan respectively. Blue and red curve shows the corresponding $Q_{T}$ of column $\mathrm{A}$ and column B.

After measuring $N_{c p c}$ and $N_{C C N}$, and $F_{n, C C N}$, we established a set of rules to find a relation between $Q_{T}$ and supersaturation. To have a reliable concentration, we considered only values when $N_{C C N}>10 / c c$ and when this value was stable for 3 consecutive measurements. Figure 4.4 graphically shows the work flow in the form of a flow chart. 


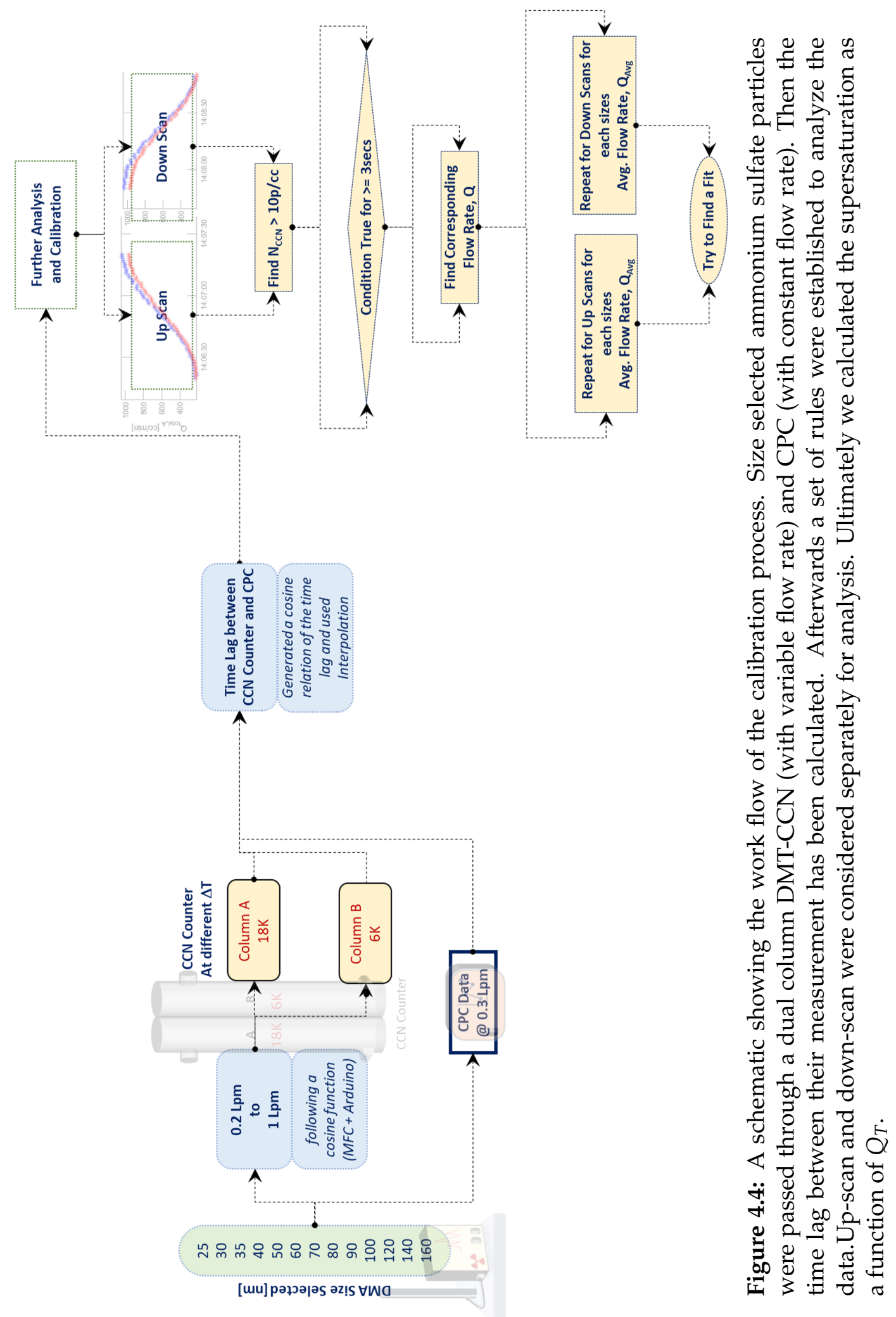




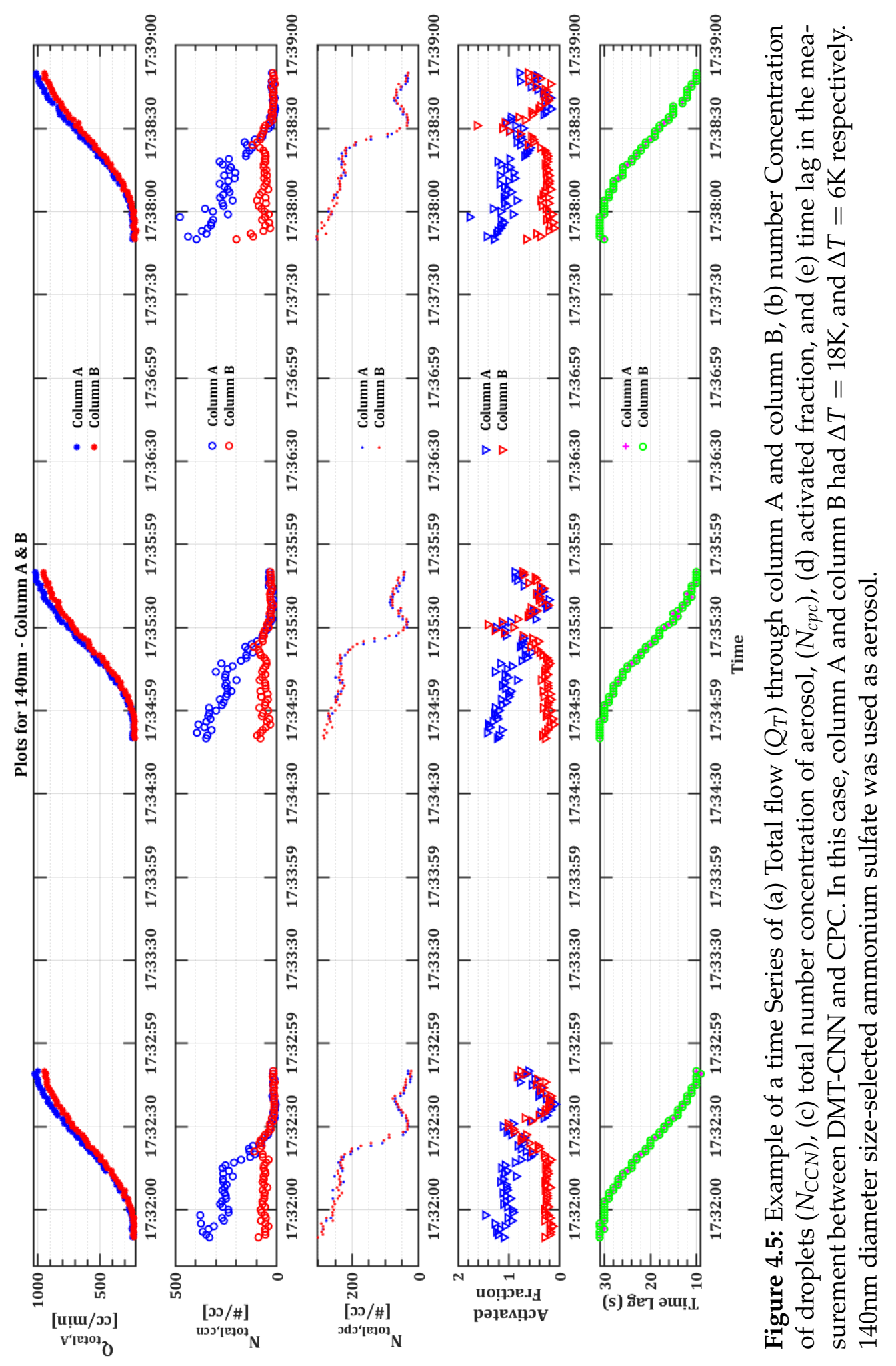


Figure 4.5 shows a time series of various parameters when $140 \mathrm{~nm}$ size selected ammonium sulfate was used. column $\mathrm{A}$ and column B was set at $\Delta T=18 \mathrm{~K}$, and $\Delta T=6 \mathrm{~K}$ respectively. Note that, smaller $N_{C C N}$ and $F_{n_{C C N}}$ for column B compared to column A, suggests that the supersaturation was only large enough to activate very few particles. In case of column A, supersaturation increased with flow and was large enough to nucleate droplets.

\subsection{Result and Discussion}

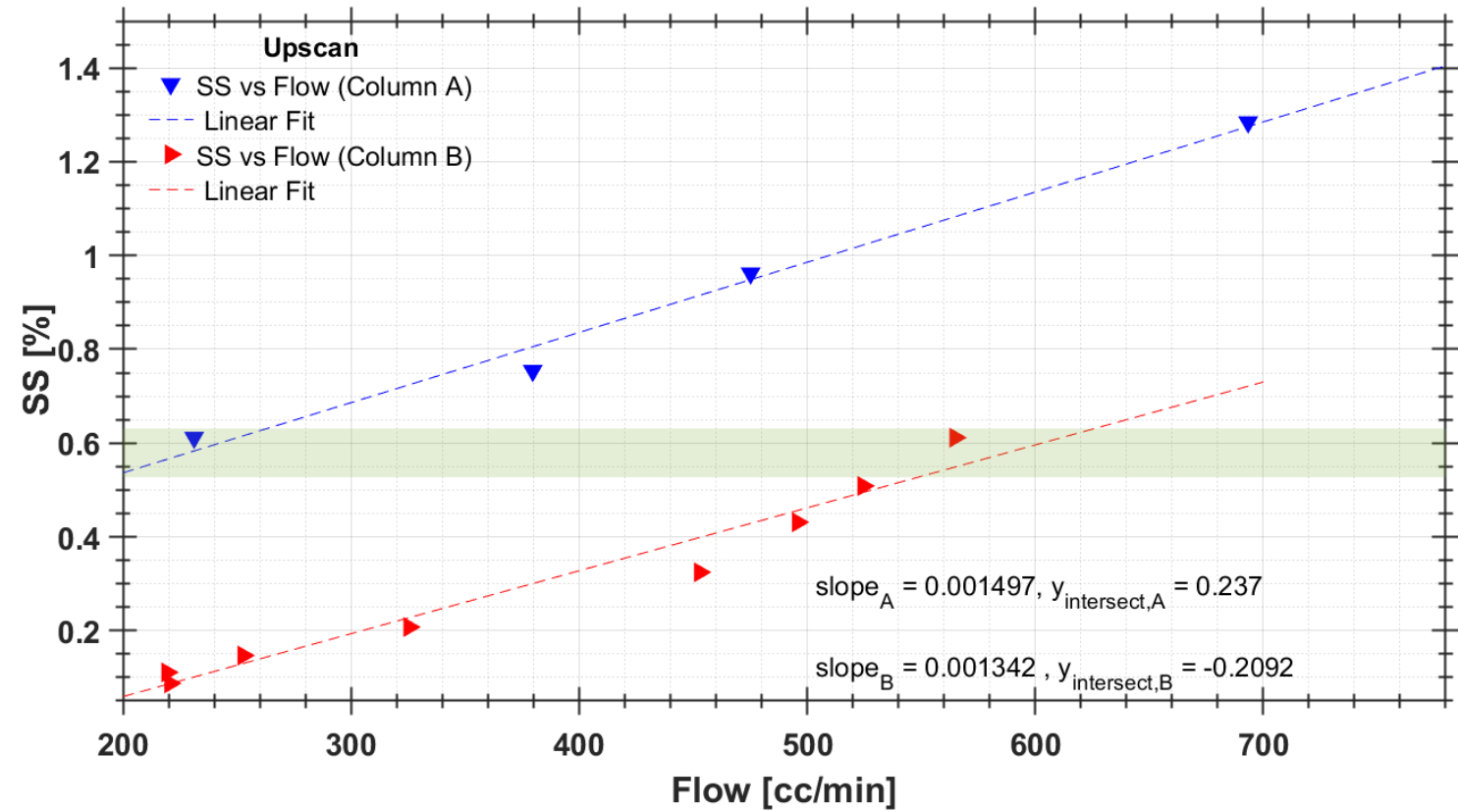

Figure 4.6: Figure showing a calibration curve for column A and column B for the upscan, delineating a relation between total flow and the resulted supersaturation. This calibration curves were obtained averaging over 3 cycles for each diameter. Column A and and Column B was set at constant thermal gradient of $18 \mathrm{~K}$ and $6 \mathrm{~K}$. The green shadow shows the overlapping supersaturation at two different $\Delta T$ and different $Q_{T}$.

Following the methodology discussed in the previous section, we have achieved the calibration curves shown in fig. 4.6 and fig. 4.7 which corresponds to up 
scan and down scan cycles, respectively. Both curves are averaged over 3 cycles for each diameter. Note that, the number of points used for the calibration is not same as the number of diameter used in the process. The primary reason behind that is either we had not achieved high enough supersaturation even with the largest flow for the smaller diameters, or the achieved supersaturation already exceeded the critical supersaturation for larger diameters.

Figure 4.6 shows the calibration curve for the up scan cycles. Blue and red triangles represents the supersaturation obtained by column A and column B respectively. Column A was set at higher $\Delta T$ than column B (18K vs $6 \mathrm{~K}$, respectively). As expected, for the same flow rate, we achieved higher supersaturation through column A than column B. Blue and red straight lines correspond to the linear fit of the data for column A and column B respectively. The green shadow shows

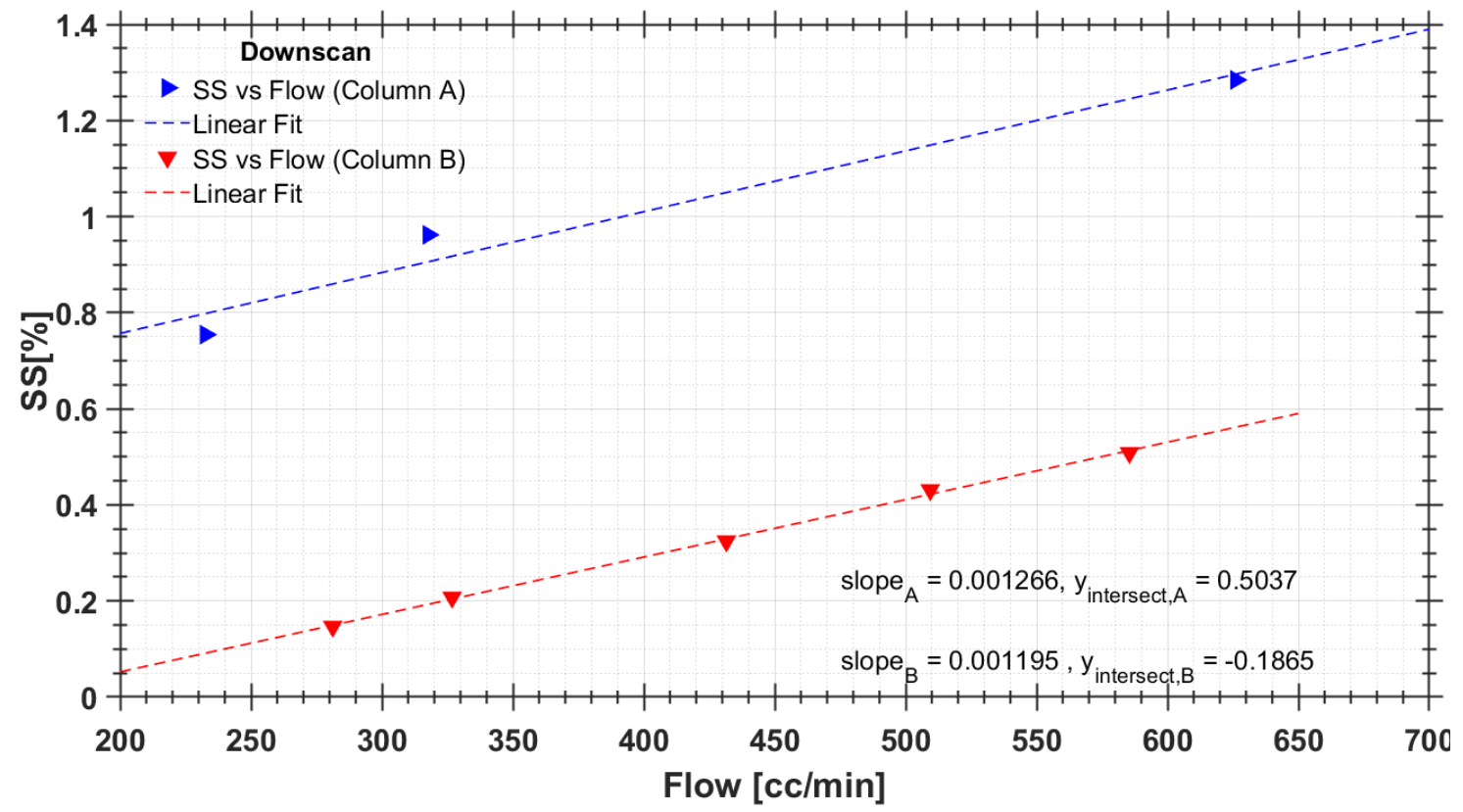

Figure 4.7: Calibration curve for column A and column B for the downscan. All the other parameters are same as the up scan case (see figure 4.6). 
the overlapping region where same supersaturation was achieved at two different flow rate and thermal gradient.

Calibration curve for the down scan cycles is shown in figure 4.7. Blue and red triangle and lines correspond to column A and column B, respectively. For down scan cycles as well, column A showed higher supersaturation than column B as expected. However, the slopes for down scan are not same as the up scan slopes. The linear fits for the down scan case also suggest that the down scan flow, in general, achieved higher supersaturation than up scan flow at the same $\Delta T$. Besides, in this case, we did not achieve the overlapping supersaturation region as we did for the up scan case.

\subsection{Summary}

A complete cycle, containing up scan (60 s), down scan (60s), and dwelling times in between ( $30 \mathrm{~s}$ each), requires in total of 3 minutes. Since the flow change is faster, a large range of supersaturation can be obtained faster than by changing $\Delta T$. The number of achievable supersaturation values is also limited.

To understand how this method would aid in the context of atmospheric measurement, let us consider field measurement data 1 . Figure 4.8 shows a time series of CCN counter measurements from the Southern Great Plains (SGP), Oklahoma. Only five discrete supersaturation can be achieved in one hour time period. Besides, a few minutes are required between the change in supersaturation to stabilize the temperature gradients.

\footnotetext{
${ }^{1}$ Uin, Janek, Cynthia Salwen, and Gunnar Senum. "Cloud Condensation Nuclei Particle Counter (AOSCCN2COLAAVG)." Atmospheric Radiation Measurement (ARM) user facility.
} 


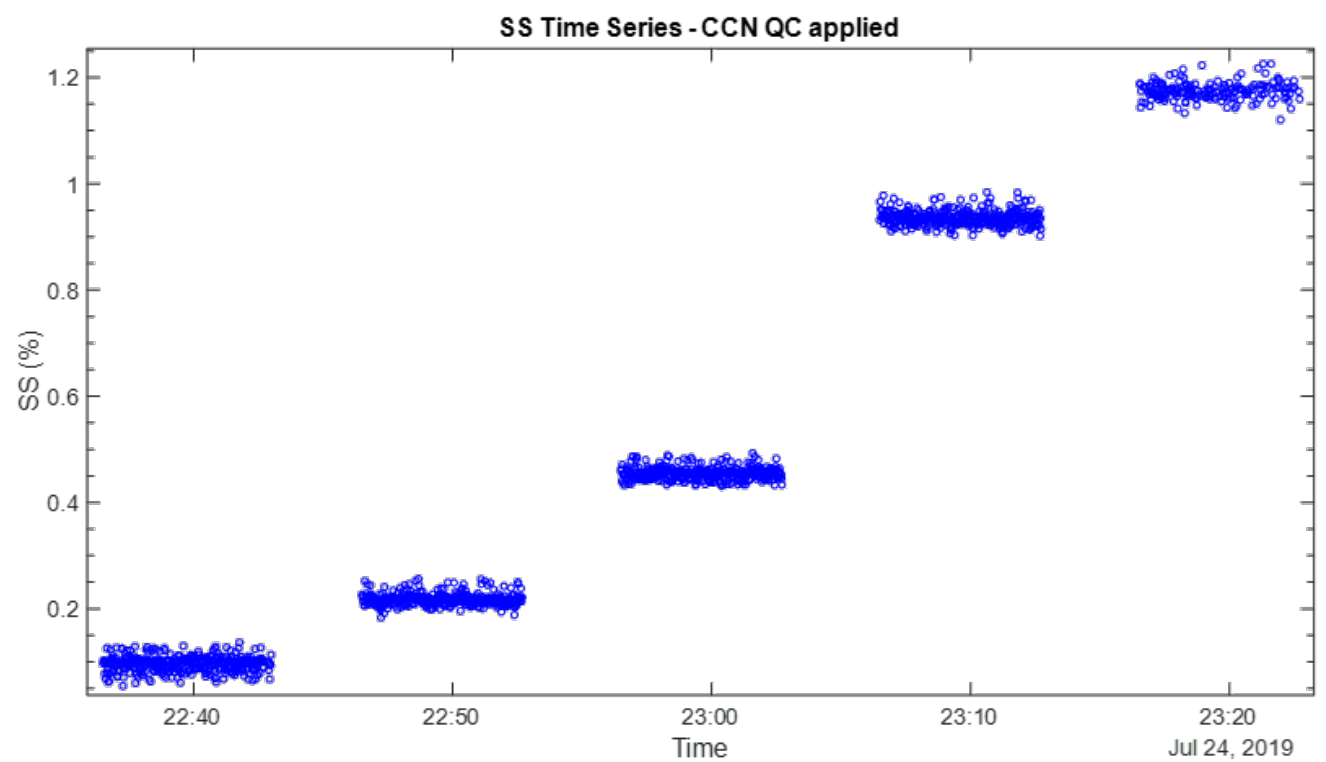

Figure 4.8: Time series of CCN counter measurement at the SGP. Quality control (QC) algorithm was applied to get a stable data set. Five supersaturation is achieved in one hour time

Therefore, for any short-term atmospheric events, we would lose information on the $\mathrm{CCN}$ activity through regular mode operation. Conversely, having properly calibrated in scanning mode, the same range of supersaturation can be achieved in a few minutes. Therefore, this mode of operation is more useful in atmospheric measurement context. 
"Like a traveller who has landed in an unknown country, I am conscious my faltering steps have extended but little beyond the starting point. All around extends the unknown, and the distance is closed in by many an Alpine peak, whose slopes will require more vigorous steps than mine to surmount. It is with reluctance I am compelled for the present to abandon the investigation. It is, however, to be hoped it will be taken up by those better fitted for the work, and that soon the roughness of the way will be levelled, the difficulties bridged, the country mapped, and its resources developed."

- Dr. John Aitken (1880)

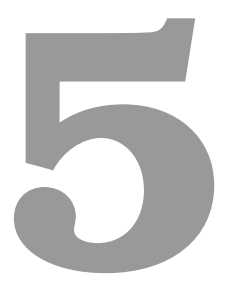

Summary 


\subsection{Summary}

Besides affecting Earth's climate through radiative balance and hydrological cycle, scavenging processes of aerosol have notable impacts on human health and the ecosystem [77, 99, 100]. Still, the scavenging processes discussed in this dissertation represent one of the most considerable uncertainties in the climate models. The motivation behind this dissertation is to improve our notions about some of these processes through planned experiments. We also look into the possible improvements that can be implemented in the available field measurement systems.

This dissertation explored how significant turbulent fluctuation is for the activation process and how much information we may lose without considering turbulence while parameterizing the activation process. One of the most commonly used parameterizations is known as Twomey Activation, where the number concentration of $\mathrm{CCN}$ is described as a function of a single peak supersaturation [27. 101]. However, in a cloudy condition, where turbulence is prevalent, we showed that considering changes due to fluctuation is necessary for a better insight into the activation process. Chapter 2.4 and the corresponding discussion in section 2.6.1 xemplifies one of the phenomena. Appreciating the effect of turbulence is essential to explain the coexistence of interstitial and cloud droplet residual of monodisperse and chemically homogeneous aerosol particles. As another example, changes in integral diameters in two different cases (same $S_{0}$ but different $\sigma_{S_{0}}$, and decreasing $S_{0}$ with same $\sigma_{S_{0}}$ ) are discussed in appendix

B. A decrease in integral diameter with decreasing saturation ratio cannot be 
explained through conventional wisdom. Keeping these examples in consideration, in chapter 2 , we attempted to introduce a relation among $S_{0}, \sigma_{S_{0}}$, and $F_{n}$. Though this relationship has its caveats, it initiates the possibility of further parameterization.

Our investigation also suggest that dry deposition of aerosol is also affected by turbulent fluctuations. The preliminary results outlined in this dissertation indicate that, in a turbulent environment, the stirred settling time is not a good approximation for the aerosol deposition time scale.

\subsection{Future Possibilities}

\section{Other Scavenging Processes}

Regarding wet scavenging processes, the scope of this dissertation is limited to activation scavenging. However, turbulence may increase the collision efficiency, and therefore, may affect the other scavenging mechanisms such as diffusion or phoretic effects [101]. The stationary cloud droplets can capture a flux of aerosol particles. This scavenging process follows simple Brownian diffusion. The efficiency of aerosol scavenging sometimes increases because of the large fall velocity of the droplets. In the cloud chamber, diffusive losses may occur both to the chamber surfaces or the cloud droplets. Chapter 3 discusses some aspects of diffusive losses to the chamber surfaces. Diffusive losses to the cloud droplets can be explored with some complementary experiments and measurements. For example, we can collect the cloud droplet residual particles and measure them using Scanning Electron Microscope (SEM) and electron dispersive x-rays (EDX) 
and quantify the presence of different materials in the collected interstitial and residuals. In addition to diffusive processes, one may consider exploring the effect of thermo- and diffisio- phoresis. These phoretic forces together may have a significant influence on in-cloud and below-cloud scavenging processes [102].

\section{Effect of Chemical Composition}

For our experiments discussed in chapter 2 , we developed a method to untwine the effect mean and fluctuation in saturation ratio on aerosol activation. Since this procedure helps to minimize the number of parameters, the impact of chemical composition on activation and the droplet size distribution could be explored independently. How the hygroscopicity of aerosol particles affects the activation

process is well known. Nonetheless, in a turbulent environment, this effect may vary. We showed that (chapter 2) turbulence can mimic the effect of chemical composition on aerosol activation. However, the relative effects of turbulence intensity and the chemical composition are yet to be explored. Furthermore, it will be interesting to explore the possibility of obtaining different turbulent regimes. Three regimes were discussed and examined in chapter 2 and Prabhakaran et al. (2020). Given the wide range of solubility of atmospheric particles, one may ask if it is possible to achieve similar regimes only by varying the chemical composition. 


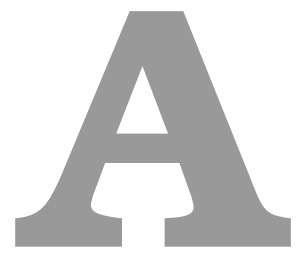

Letter of Permission 
JOHN WILEY AND SONS LICENSE

TERMS AND CONDITIONS

Aug 23, 2021

This Agreement between Michigan Technological University -- Abu Sayeed Md Shawon ("You") and John Wiley and Sons ("John Wiley and Sons") consists of your license details and the terms and conditions provided by John Wiley and Sons and Copyright Clearance

Center.

License Number

5133501076312

License date

Aug 21, 2021

Licensed Content

Publisher

John Wiley and Sons

Licensed Content

Publication

Journal of Geophysical Research: Atmospheres

Licensed Content Title

Dependence of Aerosol-Droplet Partitioning on Turbulence in a Laboratory Cloud

Licensed Content Author Will Cantrell, Raymond A. Shaw, Greg Kinney, et al

Licensed Content Date Mar 11, 2021

Licensed Content Volume 126

Licensed Content Issue 5

Licensed Content Pages 19

Type of use Dissertation/Thesis

Requestor type Author of this Wiley article

Format Print and electronic

Portion Full article 
Will you be translating? No

Title

Institution name

Expected presentation

date

Order reference number

Requestor Location

Total

Terms and Conditions
Activation Scavenging of Aerosol: Effect of Turbulence and Aerosol-Composition

Michigan Technological University

Nov 2021

Shawon_Dissertation_JGR

Michigan Technological University

1900 Stevens Dr.

Apt\#211

RICHLAND, WA 99354

United States

Attn: Michigan Technological University

EU826007151

0.00 USD

\section{TERMS AND CONDITIONS}

This copyrighted material is owned by or exclusively licensed to John Wiley \& Sons, Inc. or one of its group companies (each a"Wiley Company") or handled on behalf of a society with which a Wiley Company has exclusive publishing rights in relation to a particular work (collectively "WILEY"). By clicking "accept" in connection with completing this licensing transaction, you agree that the following terms and conditions apply to this transaction (along with the billing and payment terms and conditions established by the Copyright Clearance Center Inc., ("CCC's Billing and Payment terms and conditions"), at the time that you opened your RightsLink account (these are available at any time at http://myaccount.copyright.com). 



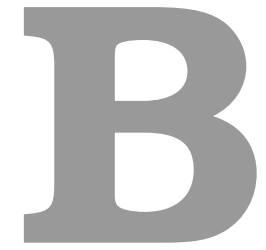

Integral Diameter 
As another perspective on the relationship between $F_{n}$ and $S_{0}$, we consider the integral diameter, $I=n_{d} \bar{D}_{w}$, defined previously (see eqn 2.2. . As discussed in section 2.5, $\tau_{c} \propto\left(n_{d} \bar{D}_{w}\right)^{-1}$. Therefore, in a steady state, an increase in $n_{d} \bar{D}_{w}$ indicates a decrease in $S_{0}$ with the caveat that $I$ does not contain information on $\sigma_{S_{0}}$. Figure B.1 shows $n_{d} \bar{D}_{w}$ as a function of $\chi_{\mathrm{H}_{2} \mathrm{O}}$ at the bottom boundary, which, as noted above, is a proxy for $S_{0}$. Two trends are immediately apparent. Initially, $n \bar{D}_{w}$ increases monotonically with decreasing $S_{0}$, as expected. However, after reaching $\chi_{\mathrm{H}_{2} \mathrm{O}}=0.93, n_{d} \bar{D}_{w}$ decreases with decreasing $S_{0}$, suggesting a change in regimes. $F_{n}$ and the size distributions of particles in the chamber are consistent with a continuously decreasing value of $S_{0}$. What happens at $\chi_{\mathrm{H}_{2} \mathrm{O}}=0.93$ ? At the highest value of $\chi$ and thus $S_{0}$, the system is in the transitional regime. The mean value of the saturation ration in the chamber is greater than $S_{c}$ for the aerosol being injected, though there are regions in the chamber where fluctuations carry the saturation ratio below $S_{c}$, as seen by the presence of the interstitial mode in the distributions from points I and II. As $S_{0}$ decreases as salt is added to the bottom boundary (decreasing $\chi_{\mathrm{H}_{2} \mathrm{O}}$ ), the mean saturation ratio in the chamber approaches $S_{c}$. Once $S_{0}<S_{c}$, cloud droplets only form and grow because of fluctuations. Smaller values of $n_{d} \bar{D}_{w}$ are associated with more subsaturated conditions where droplets only form by rare, large excursions of the saturation ratio in the chamber above $S_{c}$. 


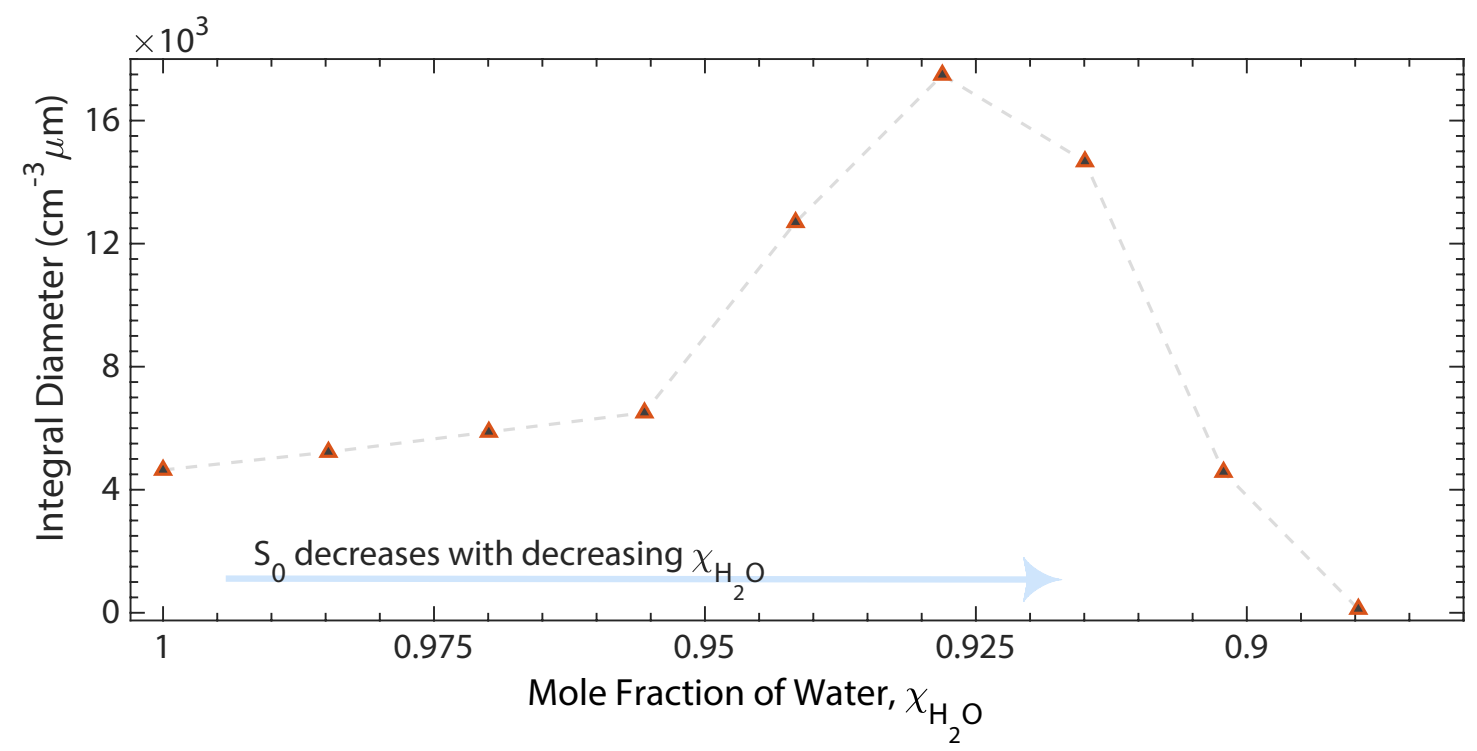

Figure B.1: $I=n_{d} \bar{D}_{w}$ as a function of the mole fraction of water, $\chi_{\mathrm{H}_{2} \mathrm{O}}$, at the bottom boundary of the chamber. High $\chi_{\mathrm{H}_{2} \mathrm{O}}$ corresponds to the high $S_{0}$ and vice versa. $n_{d} \bar{D}_{w}$ increases monotonically with decreasing $S_{0}$ until $\chi_{\mathrm{H}_{2} \mathrm{O}}=0.93$. The decrease in $I$ after that point indicates that the mean saturation ratio was either subcritical or subsaturated and activation was dominated by the fluctuations. In such cases, an increase in the integral diameter is no longer proportional to the mean saturation ratio.

Table B.1: Integral diameter and activated fraction for constant $S_{0}$.(cases I and II)

\begin{tabular}{cccr}
\hline case & $\Delta \mathrm{T}\left(\mathrm{K}^{-1}\right)$ & Integral Radius $\left(\mu \mathrm{m} \mathrm{cm}^{-3}\right)$ & Activated Fraction \\
\hline & $6 \mathrm{~K} / \mathrm{m}$ & $3.0 \times 10^{3}$ & $0.23 \pm 0.010$ \\
case I & $7 \mathrm{~K} / \mathrm{m}$ & $5.4 \times 10^{3}$ & $0.25 \pm 0.003$ \\
& $10 \mathrm{~K} / \mathrm{m}$ & $6.9 \times 10^{3}$ & $0.38 \pm 0.003$ \\
& $15 \mathrm{~K} / \mathrm{m}$ & $9.8 \times 10^{3}$ & $0.48 \pm 0.01$ \\
\hline \multirow{4}{*}{ case II } & $9 \mathrm{~K} / \mathrm{m}$ & $3.5 \times 10^{3}$ & $0.54 \pm 0.010$ \\
& $10.6 \mathrm{~K} / \mathrm{m}$ & $3.7 \times 10^{3}$ & $0.56 \pm 0.010$ \\
& $13 \mathrm{~K} / \mathrm{m}$ & $4.3 \times 10^{3}$ & $0.63 \pm 0.008$
\end{tabular}





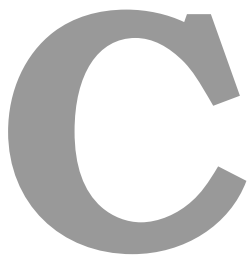

Supplementary - Chapter 2 


\section{C.1 Size-selected Sampling of Aerosols}

Figure C.1 shows the size distribution of injected aerosol. The distribution adjacent to peak A was our expected size distribution. However, since DMA classifies particles based on their electric mobility, a secondary distribution (Peak B) was observed. These particles were doubly charged, and therefore required larger diameters to have the same electric mobility. The remaining small peak on the left is actually $130 \mathrm{~nm}$ particles with two charges. Since $\sim 90 \mathrm{~nm}$ singly charged and 130nm doubly charged particles has the same electric mobility, SMPS shows those particles as $87 \mathrm{~nm}$ particles. 


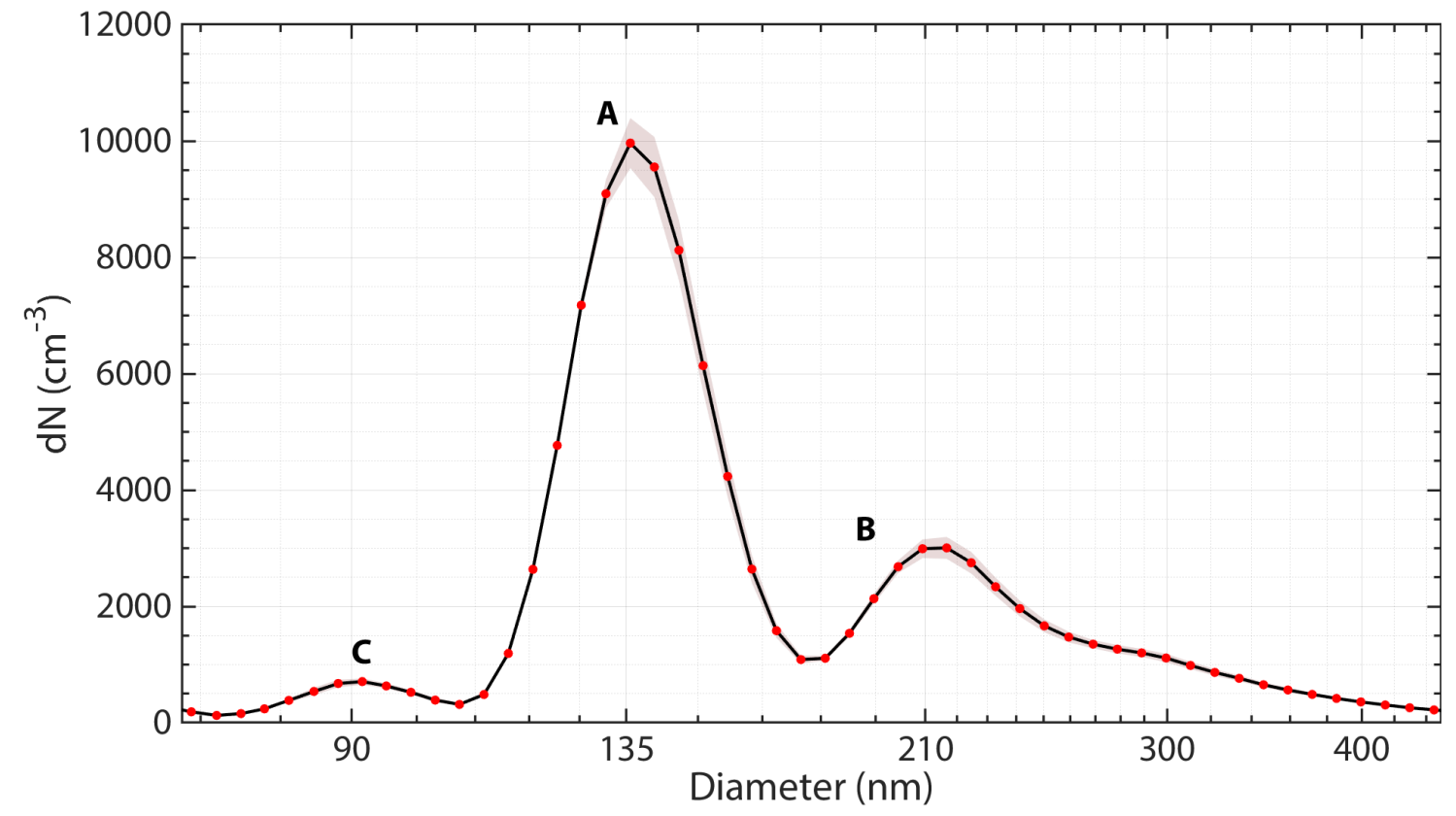

Figure C.1: Size selected $\mathrm{NaCl}$ was injected at a constant rate inside the chamber to create and sustain cloud condition. The first peak (A) and surrounding bins were the desired size bins. The secondary peak (B) and the surrounding bins were almost twice as big as the desired size bins which were selected by the DMA because of their same electric mobility. The small peak (C) on the left of the primary peak was our desired size with two charges. Note that, this figure shows the number concentration out of the atomizer. Inside the chamber this concentration has a dilution factor because of the volume difference between the cloud chamber and the atomizer. 


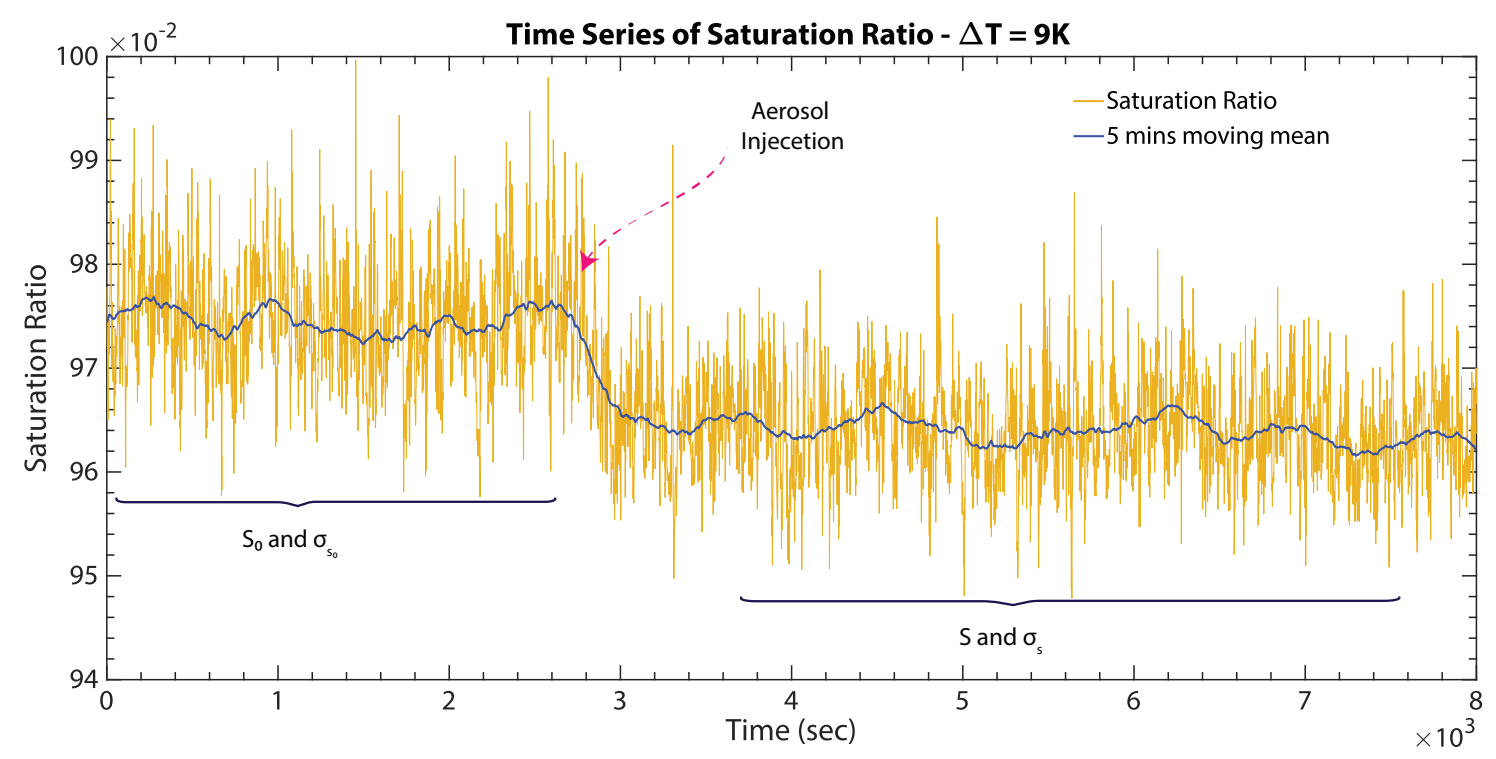

Figure C.2: Time series of saturation ratio. The saturation ratio (yellow lines) was measured using the collocated LICOR (measuring vapor pressure) and RTD (measuring temperature). Both RTD and LICOR have the sampling frequency frequency of $1 \mathrm{~Hz}$. The blue line shows 5 min moving average. Please note the drop saturation ratio approximately around 2500th second. We started injecting aerosol around that time period. The profile of saturation ratio before aerosol injection corresponds to $S_{0}, \sigma_{S_{0}}$. The saturation ratio reaches a new steady state after brief transient which is the in-cloud saturation ratio $\left(S, \sigma_{S}\right)$. 


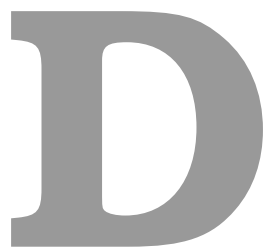

Supplementary - Chapter 3 


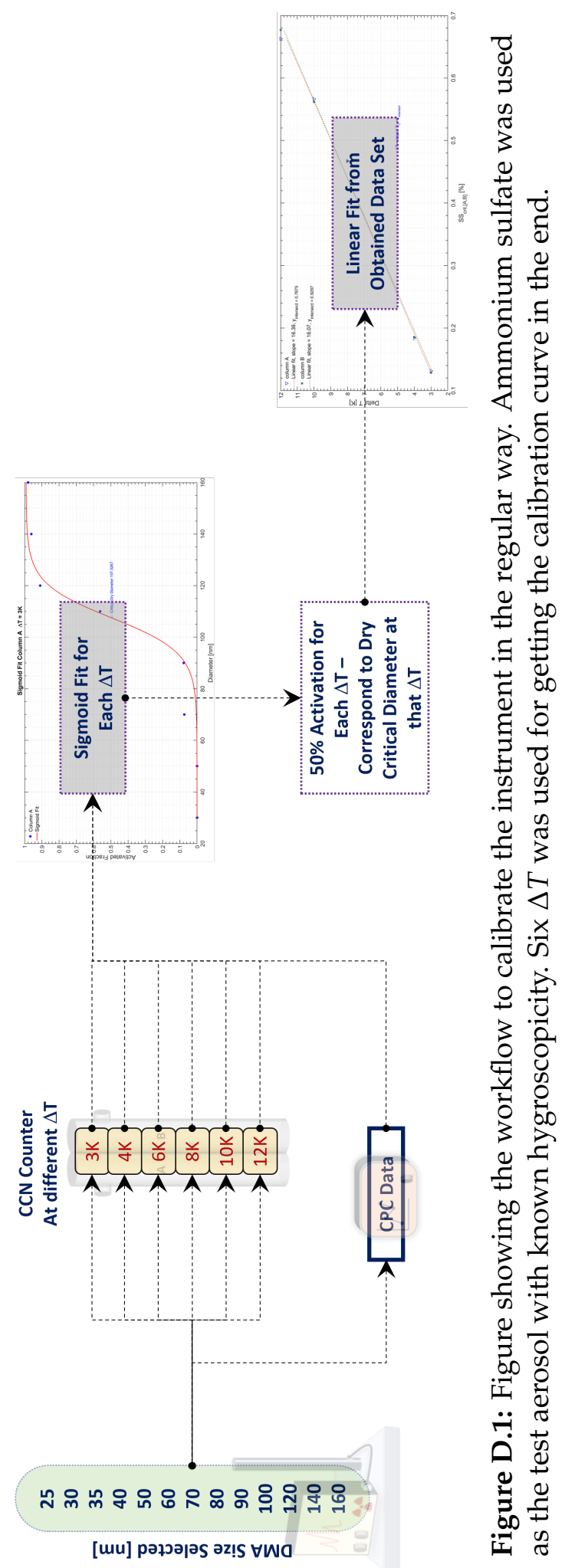




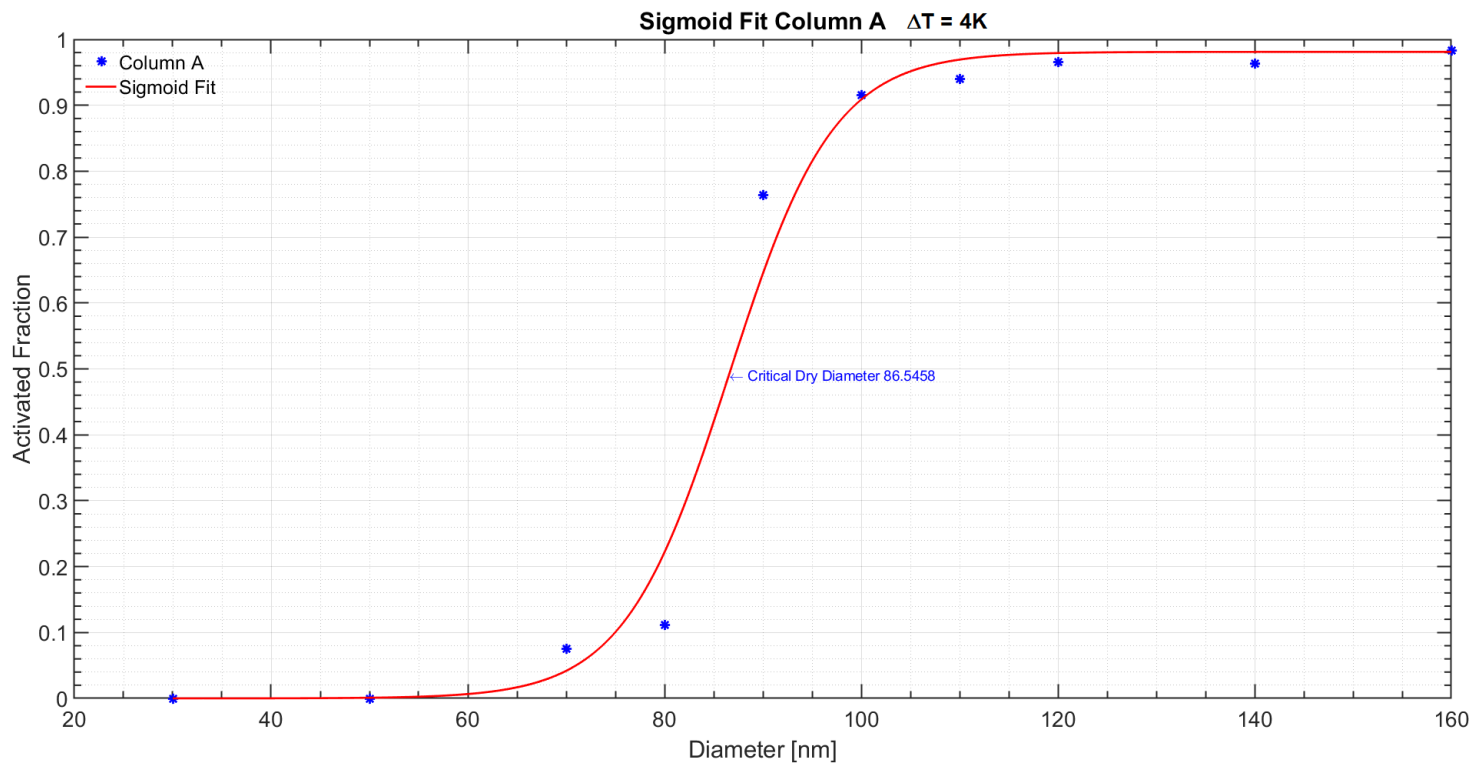

Figure D.2: Sigmoid fit for the the plot Activated Fraction vs diameter at $\Delta T$ $=4 \mathrm{~K}$. The diameters that was chosen is shown in D.1. Critical dry diameter shown in the figure was obtained by determining $50 \%$ of the maximum activated fraction. The critical supersaturation of this critical dry diameter is the supersaturation obtained by the DMT-CCNC at $4 \mathrm{~K}$ thermal gradient.

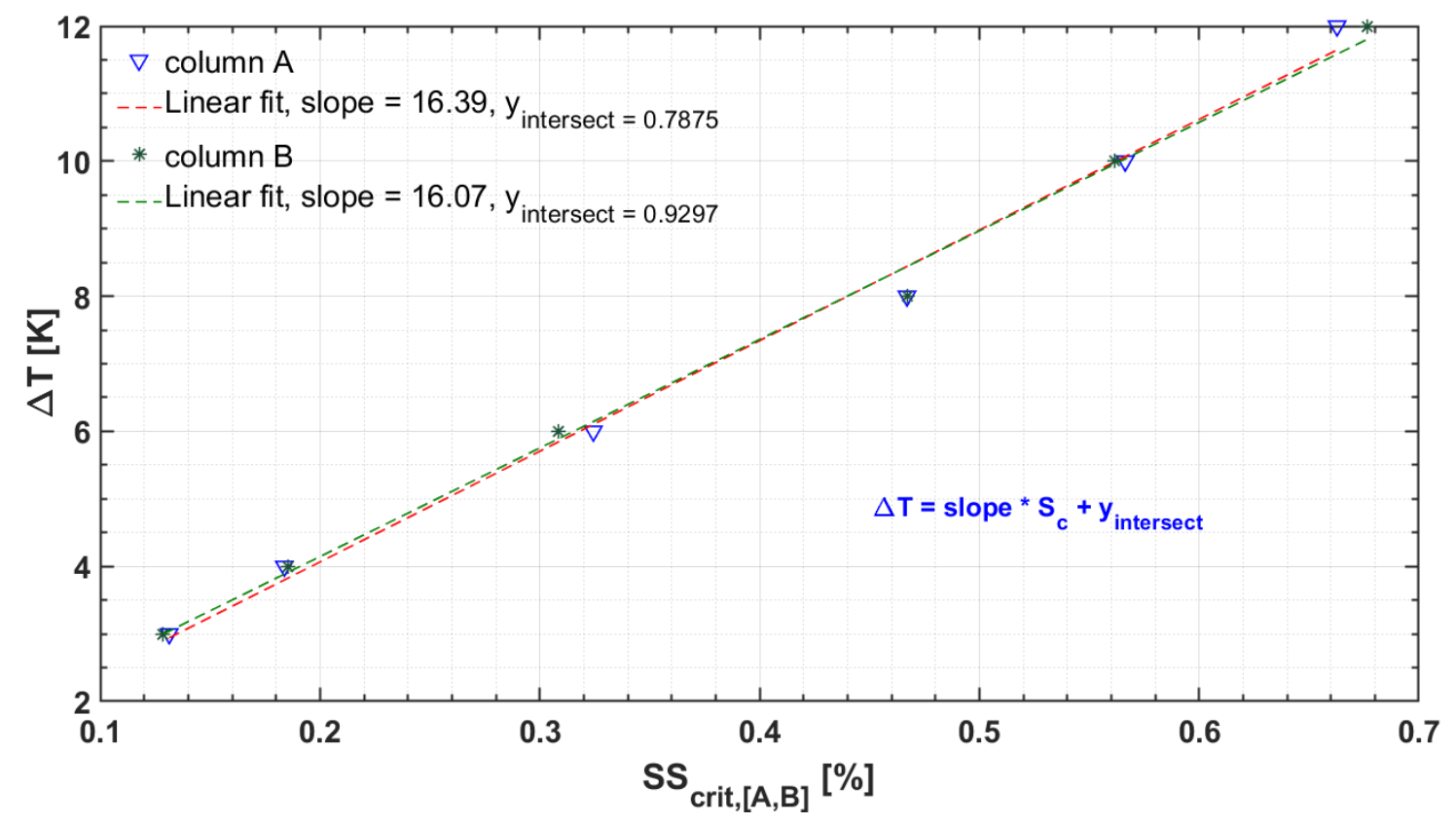

Figure D.3: Calibration curve showing $\Delta \mathrm{T}$ vs supersaturation. The reason behind using $\Delta \mathrm{T}$ vs supersaturation (not other way around) is that the operating software requires the slope and y intersect in this particular way. 



\section{References}

[1] Holger Siebert and Raymond A Shaw. Supersaturation fluctuations during the early stage of cumulus formation. Journal of the Atmospheric Sciences, 74(4):975-988, 2017.

[2] P. Reutter, H. Su, J. Trentmann, M. Simmel, D. Rose, S.S. Gunthe, H. Wernli, M. O. Andreae, and U. Pöschl. Aerosol- and updraft-limited regimes of cloud droplet formation: influence of particle number, size and hygroscopicity on the activation of cloud condensation nuclei (ccn). Atmospheric Chemistry and Physics, 9(18):7067-7080, 2009.

[3] Prasanth Prabhakaran, Abu Sayeed Md Shawon, Gregory Kinney, Subin Thomas, Will Cantrell, and Raymond A. Shaw. The role of turbulent fluctuations in aerosol activation and cloud formation. Proceedings of the $\mathrm{Na}$ tional Academy of Sciences, 2020. 
[4] Gavin Pretor-Pinney. The cloudspotter's guide: The science, history, and culture of clouds. Penguin, 2007.

[5] John Aitken. Dust, fogs, and clouds. Nature, 23(591):384-385, 1881.

[6] Peter H McMurry. A review of atmospheric aerosol measurements. Atmospheric Environment, 34(12-14):1959-1999, 2000.

[7] John Aitken. Xii.—on dust, fogs, and clouds. Transactions of the Royal Society of Edinburgh, 30(1):337-368, 1881.

[8] Hilding Köhler. The nucleus in and the growth of hygroscopic droplets. Transactions of the Faraday Society, 32(0):1152-1161, Jan 1936.

[9] Yoram J Kaufman and Didier Tanré. Effect of variations in super-saturation on the formation of cloud condensation nuclei. Nature, 369(6475):45-48, 1994.

[10] EL Zapadinsky, KK Sabelfeld, M Kulmala, BZ Gorbunov, and DM Rackimgulova. Heterogeneous nucleation in non-uniform media: $\mathrm{Nu}$ merical simulations. Journal of aerosol science, 26(8):1189-1195, 1995.

[11] Markku Kulmala, Üllar Rannik, Evgeni L Zapadinsky, and Charles F Clement. The effect of saturation fluctuations on droplet growth. Journal of aerosol science, 28(8):1395-1409, 1997.

[12] Gustavo C Abade, Wojciech W Grabowski, and Hanna Pawlowska. Broadening of cloud droplet spectra through eddy hopping: Turbulent entraining parcel simulations. Journal of the Atmospheric Sciences, 75(10):3365-3379, 2018. 
[13] Kamal Kant Chandrakar, W Cantrell, D Ciochetto, S Karki, G Kinney, and RA Shaw. Aerosol removal and cloud collapse accelerated by supersaturation fluctuations in turbulence. Geophysical Research Letters, 44(9):43594367, 2017.

[14] Ilan Koren and Graham Feingold. Aerosol-cloud-precipitation system as a predator-prey problem. Proceedings of the National Academy of Sciences, 108(30):12227-12232, 2011.

[15] S. Twomey. The influence of pollution on the shortwave albedo of clouds. Journal of the Atmospheric Sciences, 34(7):1149-1152, 1977.

[16] B. A. Albrecht. Aerosols, cloud microphysics, and fractional cloudiness. Science, 245(4923):1227-1230, 1989.

[17] Robert Pincus and Marcia B. Baker. Effect of precipitation on the albedo susceptibility of clouds in the marine boundary layer. Nature, 372:250, 1994.

[18] James Haywood and Olivier Boucher. Estimates of the direct and indirect radiative forcing due to tropospheric aerosols: A review. Reviews of geophysics, 38(4):513-543, 2000.

[19] Andrea I Flossmann, WD Hall, and HR Pruppacher. A theoretical study of the wet removal of atmospheric pollutants. part i: The redistribution of aerosol particles captured through nucleation and impaction scavenging by growing cloud drops. Journal of Atmospheric Sciences, 42(6):583-606, 1985. 
[20] Leiming Zhang, Sunling Gong, Jacob Padro, and Len Barrie. A sizesegregated particle dry deposition scheme for an atmospheric aerosol module. Atmospheric environment, 35(3):549-560, 2001.

[21] VCPJ Ramanathan, PJ Crutzen, JT Kiehl, and Daniel Rosenfeld. Aerosols, climate, and the hydrological cycle. science, 294(5549):2119-2124, 2001.

[22] Daniel Rosenfeld, Steven Sherwood, Robert Wood, and Leo Donner. Climate effects of aerosol-cloud interactions. Science, 343(6169):379-380, 2014.

[23] Jagdish Chandra Kuniyal and Raj Paul Guleria. The current state of aerosol-radiation interactions: a mini review. Journal of Aerosol Science, 130:45-54, 2019.

[24] S. Twomey. Pollution and the planetary albedo. Atmospheric Environment (1967), 8(12):1251-1256, 1974.

[25] U. Lohmann and J. Feichter. Global indirect aerosol effects: a review. Atmospheric Chemistry and Physics, 5(3):715-737, 2005.

[26] Sho Ohata, Nobuhiro Moteki, Tatsuhiro Mori, Makoto Koike, and Yutaka Kondo. A key process controlling the wet removal of aerosols: new observational evidence. Scientific reports, 6(1):1-9, 2016.

[27] Sean Twomey. The nuclei of natural cloud formation part ii: The supersaturation in natural clouds and the variation of cloud droplet concentration. Geofisica pura e applicata, 43(1):243-249, 1959. 
[28] Steven J Ghan, Hayder Abdul-Razzak, Athanasios Nenes, Yi Ming, Xiaohong Liu, Mikhail Ovchinnikov, Ben Shipway, Nicholas Meskhidze, Jun $\mathrm{Xu}$, and Xiangjun Shi. Droplet nucleation: Physically-based parameterizations and comparative evaluation. Journal of Advances in Modeling Earth Systems, 3(4), 2011.

[29] Florian Ditas, Raymond A Shaw, Holger Siebert, Martin Simmel, Birgit Wehner, and Alfred Wiedensohler. Aerosols-cloud microphysicsthermodynamics-turbulence: evaluating supersaturation in a marine stratocumulus cloud. Atmospheric Chemistry and Physics, 12(5):2459-2468, 2012.

[30] MJ Manton. On the broadening of a droplet distribution by turbulence near cloud base. Quarterly Journal of the Royal Meteorological Society, 105(446):899-914, 1979.

[31] William A. Cooper. Effects of variable droplet growth histories on droplet size distributions. part i: Theory. Journal of the Atmospheric Sciences, 46(10):1301--1311, 1988.

[32] Alexei Korolev, Mark Pinsky, and Alex Khain. A new mechanism of droplet size distribution broadening during diffusional growth. Journal of the atmospheric sciences, 70(7):2051-2071, 2013.

[33] Kamal Kant Chandrakar, Will Cantrell, Kelken Chang, David Ciochetto, Dennis Niedermeier, Mikhail Ovchinnikov, Raymond A Shaw, and Fan Yang. Aerosol indirect effect from turbulence-induced broadening of cloud-droplet size distributions. Proceedings of the National Academy of Sciences, 113(50):14243-14248, 2016. 
[34] Raymond A Shaw, Walter C Reade, Lance R Collins, and Johannes Verlinde. Preferential concentration of cloud droplets by turbulence: Effects on the early evolution of cumulus cloud droplet spectra. Journal of the atmospheric sciences, 55(11):1965-1976, 1998.

[35] PA Vaillancourt, MK Yau, P Bartello, and Wojciech W Grabowski. Microscopic approach to cloud droplet growth by condensation. part ii: Turbulence, clustering, and condensational growth. Journal of the atmospheric sciences, 59(24):3421-3435, 2002.

[36] K Chang, J Bench, M Brege, Will Cantrell, Kamal Kant Chandrakar, David Ciochetto, Claudio Mazzoleni, LR Mazzoleni, Dennis Niedermeier, and RA Shaw. A laboratory facility to study gas-aerosol-cloud interactions in a turbulent environment: The $\pi$ chamber. Bulletin of the American Meteorological Society, 97(12):2343-2358, 2016.

[37] Kamal Kant Chandrakar, Will Cantrell, A.B. Kostinski, and R.A. Shaw. Dispersion aerosol indirect effect in turbulent clouds: Laboratory measurements of effective radius. Geophysical research letters, 45(19):10-738, 2018.

[38] Kamal Kant Chandrakar, Will Cantrell, and Raymond A Shaw. Influence of turbulent fluctuations on cloud droplet size dispersion and aerosol indirect effects. Journal of the atmospheric sciences, 75(9):3191-3209, 2018.

[39] N Desai, Kamal Kant Chandrakar, K Chang, W Cantrell, and RA Shaw. Influence of microphysical variability on stochastic condensation in a turbulent laboratory cloud. Journal of the Atmospheric Sciences, 75(1):189-201, 2018. 
[40] Subin Thomas, Mikhail Ovchinnikov, Fan Yang, Dennis van der Voort, Will Cantrell, Steven K Krueger, and Raymond A Shaw. Scaling of an atmospheric model to simulate turbulence and cloud microphysics in the pi chamber. Journal of Advances in Modeling Earth Systems, 11(7):1981-1994, 2019.

[41] Roddy Rhodes Rogers and Man Kong Yau. A short course in cloud physics. International series in natural philosophy. Butterwoth-Heinemann, 3. ed., reprint edition, 1996.

[42] Alexei Korolev and Ilia P Mazin. Supersaturation of water vapor in clouds. Journal of the atmospheric sciences, 60(24):2957-2974, 2003.

[43] C. F. Bohren and B. A. Albrecht. Atmospheric Thermodynamics. Oxford University Press, New York, 1998.

[44] M. D. Petters and S. M. Kreidenweis. A single parameter representation of hygroscopic growth and cloud condensation nucleus activity. Atmospheric Chemistry and Physics, 7(8):1961-1971, 2007.

[45] M. D. Petters and S. M. Kreidenweis. A single parameter representation of hygroscopic growth and cloud condensation nucleus activity - part 2: Including solubility. Atmospheric Chemistry and Physics, 8(20):6273-6279, 2008.

[46] Benjamin J. Mullins, Daniel Kampa, and Gerhard Kasper. Comment on "performance evaluation of 3 optical particle counters with an efficient multimodal calibration method" (heim et al., 2008)—performance of improved counter. Journal of Aerosol Science, 49:48-50, 2012. 
[47] John H Seinfeld and Spyros N Pandis. Atmospheric chemistry and physics: from air pollution to climate change. John Wiley \& Sons, 2012.

[48] N. V. Gillani, S. E. Schwartz, W. R. Leaitch, J. W. Strapp, and G. A. Isaac. Field observations in continental stratiform clouds: Partitioning of cloud particles between droplets and unactivated interstitial aerosols. Journal of Geophysical Research: Atmospheres, 100(D9):18687-18706, 1995.

[49] Dennis Niedermeier, Kelken Chang, Will Cantrell, Kamal Kant Chandrakar, David Ciochetto, and Raymond A Shaw. Observation of a link between energy dissipation rate and oscillation frequency of the large-scale circulation in dry and moist rayleigh-bénard turbulence. Physical Review Fluids, 3(8):083501, 2018.

[50] Francesca Chillà and Joerg Schumacher. New perspectives in turbulent rayleigh-bénard convection. The European Physical Journal E, 35(7):1-25, 2012.

[51] Bart Verheggen, Julie Cozic, Ernest Weingartner, Keith Bower, Stephan Mertes, Paul Connolly, Martin Gallagher, Michael Flynn, Tom Choularton, and Urs Baltensperger. Aerosol partitioning between the interstitial and the condensed phase in mixed-phase clouds. Journal of Geophysical Research: Atmospheres, 112(D23), 2007.

[52] S. Henning, S. Bojinski, K. Diehl, S. Ghan, S. Nyeki, E. Weingartner, S. Wurzler, and U. Baltensperger. Aerosol partitioning in natural mixed-phase clouds. Geophysical Research Letters, 31(6), 2004. 
[53] C. H. Twohy, J. R. Anderson, D. W. Toohey, M. Andrejczuk, A. Adams, M. Lytle, R. C. George, R. Wood, P. Saide, S. Spak, P. Zuidema, and D. Leon. Impacts of aerosol particles on the microphysical and radiative properties of stratocumulus clouds over the southeast pacific ocean. Atmospheric Chemistry and Physics, 13(5):2541-2562, 2013.

[54] Dennis Niedermeier, Jens Voigtländer, Silvio Schmalfuß, Daniel Busch, Jörg Schumacher, Raymond A Shaw, and Frank Stratmann. Characterization and first results from LACIS-T: a moist-air wind tunnel to study aerosol-cloud-turbulence interactions. Atmos. Measure. Tech., 13(4):2015$2015,2020$.

[55] Jingyi Chen, Yangang Liu, Minghua Zhang, and Yiran Peng. New understanding and quantification of the regime dependence of aerosol-cloud interaction for studying aerosol indirect effects. Geophysical Research Letters, 43(4):1780-1787, 2016.

[56] Holger Siebert, Harald Franke, Katrin Lehmann, Rolf Maser, Ewe Wei Saw, Dieter Schell, Raymond A. Shaw, and Manfred Wendisch. Probing finescale dynamics and microphysics of clouds with helicopter-borne measurements. Bulletin of the American Meteorological Society, 87(12):1727-1738, 2006.

[57] Holger Siebert, Katrin Lehmann, and Manfred Wendisch. Observations of small-scale turbulence and energy dissipation rates in the cloudy boundary layer. Journal of the Atmospheric Sciences, 63(5):1451-1466, 2006. 
[58] Wojciech W. Grabowski and Lian-Ping Wang. Growth of cloud droplets in a turbulent environment. Annual Review of Fluid Mechanics, 45(1):293-324, 2013.

[59] Fan Yang, Robert McGraw, Edward P. Luke, Damao Zhang, Pavlos Kollias, and Andrew M. Vogelmann. A new approach to estimate supersaturation fluctuations in stratocumulus cloud using ground-based remote-sensing measurements. Atmospheric Measurement Techniques, 12(11):5817-5828, 2019.

[60] Ewe-Wei Saw, Juan PLC Salazar, Lance R Collins, and Raymond A Shaw. Spatial clustering of polydisperse inertial particles in turbulence: I. comparing simulation with theory. New Journal of Physics, 14(10):105030, 2012.

[61] Ewe-Wei Saw, Raymond A Shaw, Juan PLC Salazar, and Lance R Collins. Spatial clustering of polydisperse inertial particles in turbulence: Ii. comparing simulation with experiment. New Journal of Physics, 14(10):105031, 2012.

[62] H Siebert, S Gerashchenko, A Gylfason, K Lehmann, LR Collins, RA Shaw, and Z Warhaft. Towards understanding the role of turbulence on droplets in clouds: in situ and laboratory measurements. Atmospheric research, 97(4):426-437, 2010.

[63] RC Srivastava. Growth of cloud drops by condensation: A criticism of currently accepted theory and a new approach. Journal of the atmospheric sciences, 46(7):869-887, 1989. 
[64] Jingyi Chen, Yangang Liu, Minghua Zhang, and Yiran Peng. Height dependency of aerosol-cloud interaction regimes. Journal of Geophysical Research: Atmospheres, 123(1):491-506, 2018.

[65] Tom Goren, Jan Kazil, Fabian Hoffmann, Takanobu Yamaguchi, and Graham Feingold. Anthropogenic air pollution delays marine stratocumulus breakup to open cells. Geophysical Research Letters, 46(23):14135-14144, 2019.

[66] Tom Goren and Daniel Rosenfeld. Extensive closed cell marine stratocumulus downwind of europe-a large aerosol cloud mediated radiative effect or forcing? J. Geophys. Res., 120(12):6098-6116, 2015.

[67] Robert Wood. Rate of loss of cloud droplets by coalescence in warm clouds. Journal of Geophysical Research: Atmospheres, 111(D21), 2006.

[68] J.R. Pierce, B. Croft, J.K. Kodros, S.D. D'Andrea, and R.V. Martin. The importance of interstitial particle scavenging by cloud droplets in shaping the remote aerosol size distribution and global aerosol-climate effects. Atmospheric Chemistry and Physics, 15:6147-6158, 2015.

[69] Kuan-Ting O, Robert Wood, and Christopher S. Bretherton. Ultraclean Layers and Optically Thin Clouds in the Stratocumulus-to-Cumulus Transition. Part II: Depletion of Cloud Droplets and Cloud Condensation Nuclei through Collision-Coalescence. Journal of the Atmospheric Sciences, 75(5):1653-1673, 2018. 
[70] James G Hudson and Paul R Frisbie. Cloud condensation nuclei near marine stratus. Journal of Geophysical Research: Atmospheres, 96(D11):2079520808, 1991.

[71] Ken W Nicholson. The dry deposition of small particles: a review of experimental measurements. Atmospheric Environment (1967), 22(12):2653-2666, 1988.

[72] Delphine K Farmer, Erin K Boedicker, and Holly M DeBolt. Dry deposition of atmospheric aerosols: Approaches, observations, and mechanisms. Annual Review of Physical Chemistry, 72:375-397, 2021.

[73] Allen H Goldstein and Ian E Galbally. Known and unexplored organic constituents in the earth's atmosphere. Environmental science \& technology, 41(5):1514-1521, 2007.

[74] ACK Lai. Particle deposition indoors: a review. Indoor air, 12(4):211-214, 2002.

[75] Chantal Darquenne. Deposition mechanisms. Journal of aerosol medicine and pulmonary drug delivery, 33(4):181-185, 2020.

[76] James G Crump and John H Seinfeld. Turbulent deposition and gravitational sedimentation of an aerosol in a vessel of arbitrary shape. Journal of Aerosol Science, 12(5):405-415, 1981.

[77] SH Park, HO Kim, YT Han, SB Kwon, and KW Lee. Wall loss rate of polydispersed aerosols. Aerosol Science E Technology, 35(3):710-717, 2001. 
[78] Ethan W Emerson, Anna L Hodshire, Holly M DeBolt, Kelsey R Bilsback, Jeffrey R Pierce, Gavin R McMeeking, and Delphine K Farmer. Revisiting particle dry deposition and its role in radiative effect estimates. Proceedings of the National Academy of Sciences, 117(42):26076-26082, 2020.

[79] A Fuchs and CN Davies. Aerosol mechanics. Book Reviews: The Mechanics of Aerosols). Science, 146(3647):1033-1034, 1964.

[80] William C Hinds. Aerosol technology: properties, behavior, and measurement of airborne particles. John Wiley \& Sons, 2012.

[81] M Giardina and P Buffa. A new approach for modeling dry deposition velocity of particles. Atmospheric Environment, 180:11-22, 2018.

[82] AW Harrison. Quiescent boundary layer thickness in aerosol enclosures under convective stirring conditions. Journal of Colloid and Interface Science, 69(3):563-570, 1979.

[83] M.O. Andreae and D. Rosenfeld. Aerosol-cloud-precipitation interactions. part 1. the nature and sources of cloud-active aerosols. Earth-Science Reviews, 89(1-2):13-41, Jul 2008.

[84] S. Lance, A. Nenes, J. Medina, and J. N. Smith. Mapping the operation of the dmt continuous flow ccn counter. Aerosol Science and Technology, 40(4):242-254, Apr 2006.

[85] Pradeep Saxena and Lynn M Hildemann. Water-soluble organics in atmospheric particles: A critical review of the literature and application of thermodynamics to identify candidate compounds. Journal of atmospheric chemistry, 24(1):57-109, 1996. 
[86] S Twomey. Measurements of natural cloud nuclei. J. Rech. Atmos, 1:101105, 1963.

[87] GG Lala and JE Jiusto. An automatic light scattering ccn counter. Journal of Applied Meteorology and Climatology, 16(4):413-418, 1977.

[88] AM Sinnarwalla and DJ Alofs. A cloud nucleus counter with long available growth time. Journal of Applied Meteorology, 12(5):831-835, 1973.

[89] GC Roberts and Athanasios Nenes. A continuous-flow streamwise thermal-gradient ccn chamber for atmospheric measurements. Aerosol Science and Technology, 39(3):206-221, 2005.

[90] Patrick Y Chuang, Athanasios Nenes, James N Smith, Richard C Flagan, and John H Seinfeld. Design of a ccn instrument for airborne measurement. Journal of Atmospheric and Oceanic Technology, 17(8):1005-1019, 2000.

[91] Athanasios Nenes, Patrick Y Chuang, Richard C Flagan, and John H Seinfeld. A theoretical analysis of cloud condensation nucleus (ccn) instruments. Journal of Geophysical Research: Atmospheres, 106(D4):3449-3474, 2001.

[92] WA Hoppel. Measurement of the size distribution and ccn supersaturation spectrum of submicron aerosols over the ocean. Journal of Atmospheric Sciences, 36(10):2006-2015, 1979.

[93] R Leaitch and WJ Megaw. The diffusion tube; a cloud condensation nucleus counter for use below $0.3 \%$ supersaturation. Journal of Aerosol Science, 13(4):297-319, 1982. 
[94] Diana Rose, SS Gunthe, E Mikhailov, GP Frank, Ulrike Dusek, Meinrat O Andreae, and Ulrich Pöschl. Calibration and measurement uncertainties of a continuous-flow cloud condensation nuclei counter (dmt-cenc): $\mathrm{Ccn}$ activation of ammonium sulfate and sodium chloride aerosol particles in theory and experiment. Atmospheric Chemistry and Physics, 8(5):1153-1179, 2008.

[95] Richard H Moore and Athanasios Nenes. Scanning flow ccn analysis-a method for fast measurements of ccn spectra. Aerosol Science and Technology, 43(12):1192-1207, 2009.

[96] Sarah R Suda, Markus D Petters, Geoffrey K Yeh, Christen Strollo, Aiko Matsunaga, Annelise Faulhaber, Paul J Ziemann, Anthony J Prenni, Christian M Carrico, Ryan C Sullivan, et al. Influence of functional groups on organic aerosol cloud condensation nucleus activity. Environmental science E technology, 48(17):10182-10190, 2014.

[97] Yichuan Wang, Yannian Zhu, Minghuai Wang, Daniel Rosenfeld, Yang Gao, Xiaohong Yao, Lifang Sheng, Avichay Efraim, and Juntao Wang. Validation of satellite-retrieved ccn based on a cruise campaign over the polluted northwestern pacific ocean. Atmospheric Research, page 105722, 2021.

[98] Lynn M Russell, Armin Sorooshian, John H Seinfeld, Bruce A Albrecht, Athanasios Nenes, Lars Ahlm, Yi-Chun Chen, Matthew Coggon, Jill S Craven, Richard C Flagan, et al. Eastern pacific emitted aerosol cloud experiment. Bulletin of the American Meteorological Society, 94(5):709-729, 2013. 
[99] Sonia M Kreidenweis, Markus Petters, and Ulrike Lohmann. 100 years of progress in cloud physics, aerosols, and aerosol chemistry research. Meteorological Monographs, 59:11-1, 2019.

[100] Olivier Boucher. Atmospheric aerosols. In Atmospheric Aerosols, pages 924. Springer, 2015.

[101] Hans R Pruppacher and James D Klett. Microphysics of Clouds and Precipitation: Reprinted 1980. Springer Science \& Business Media, 2012.

[102] Gianni Santachiara, Franco Prodi, and Franco Belosi. Atmospheric aerosol scavenging processes and the role of thermo-and diffusio-phoretic forces. Atmospheric research, 128:46-56, 2013.

[103] Andrew H Bell and Douglas P Munoz. Activity in the superior colliculus reflects dynamic interactions between voluntary and involuntary influences on orienting behaviour. Eur. J. Neurosci., 28(8):1654-1660, 2008.

[104] George S Kell. Density, thermal expansivity, and compressibility of liquid water from 0 . deg. to 150. deg.. correlations and tables for atmospheric pressure and saturation reviewed and expressed on 1968 temperature scale. Journal of Chemical and Engineering Data, 20(1):97-105, 1975.

[105] Boris I Shraiman and Eric D Siggia. Scalar turbulence. Nature, 405(6787):639-646, 2000.

[106] E. Hammer, M. Gysel, G. C. Roberts, T. Elias, J. Hofer, C. R. Hoyle, N. Bukowiecki, J.-C. Dupont, F. Burnet, U. Baltensperger, and et al. Size-dependent particle activation properties in fog during the 
parisfog 2012/13 field campaign. Atmospheric Chemistry and Physics, 14(19):10517-10533, 2014.

[107] Marie Mazoyer, Frédéric Burnet, Cyrielle Denjean, Gregory C. Roberts, Martial Haeffelin, Jean-Charles Dupont, and Thierry Elias. Experimental study of the aerosol impact on fog microphysics. Atmospheric Chemistry and Physics, 19(7):4323-4344, 2019.

[108] J. E. Boulter, D. J. Cziczo, A. M. Middlebrook, D. S. Thomson, and D. M. Murphy. Design and performance of a pumped counterflow virtual impactor. Aerosol Science and Technology, 40(11):969-976, 2006.

[109] Gourihar Kulkarni, Mikhail Pekour, Armin Afchine, Daniel M Murphy, and Daniel J Cziczo. Comparison of experimental and numerical studies of the performance characteristics of a pumped counterflow virtual impactor. Aerosol science and technology, 45(3):382-392, 2011.

[110] Jiwen Fan, Yuan Wang, Daniel Rosenfeld, and Xiaohong Liu. Review of aerosol-cloud interactions: Mechanisms, significance, and challenges. Journal of the Atmospheric Sciences, 73(11):4221-4252, 2016.

[111] Nicolas Bellouin, Johannes Quaas, Edward Gryspeerdt, Stefan Kinne, Philip Stier, Duncan Watson-Parris, Olivier Boucher, Ken S Carslaw, Matthew Christensen, A-L Daniau, et al. Bounding global aerosol radiative forcing of climate change. Reviews of Geophysics, 58(1):e2019RG000660, 2020

[112] Olivier Boucher, David Randall, Paulo Artaxo, Christopher Bretherton, Gragam Feingold, Piers Forster, VM Kerminen, Yutaka Kondo, Hong Liao, 
Ulrike Lohmann, P. Rasch, S.K. Satheesh, S. Sherwood, B. Stevens, and X.Y. Zhang. Clouds and aerosols. pages 571-657, 2013.

[113] G. Mcfiggans, P. Artaxo, Urs Baltensperger, H. Coe, M. C. Facchini, G. Feingold, S. Fuzzi, M. Gysel, A. Laaksonen, U. Lohmann, T. F. Mentel, D. M. Murphy, C. D. O'Dowd, J. R. Snider, and E. Weingartner. The effect of physical and chemical aerosol properties on warm cloud droplet activation. Atmospheric Chemistry and Physics Discussions, 5(5):8507-8647, 2005.

[114] Eberhard Bodenschatz, Szymon P Malinowski, Raymond A Shaw, and Frank Stratmann. Can we understand clouds without turbulence? Science, 327(5968):970-971, 2010.

[115] Oleg A Alduchov and Robert E Eskridge. Improved magnus form approximation of saturation vapor pressure. Journal of applied meteorology, 35(4):601-609, 1996.

[116] Natalia Babkovskaia, Ullar Rannik, Vaughan Phillips, Holger Siebert, Birgit Wehner, and Michael Boy. A dns study of aerosol and smallscale cloud turbulence interaction. Atmospheric Chemistry and Physics, 16(12):7889-7898, 2016.

[117] A Hallberg, KJ Noone, and JA Ogren. Aerosol particles and clouds: which particles form cloud droplets? Tellus B: Chemical and Physical Meteorology, 50(1):59-75, 1998.

[118] Kevin J Noone, John A Ogren, Anneli Hallberg, Jost Heintzenberg, Johan Strom, Hans-Christen Hansson, Birgitta Svenningsson, Alfred Wiedensohler, Sandro Fuzzi, Maria Christina Facchini, et al. Changes in aerosol 
size- and phase distributions due to physical and chemical processes in fog. Tellus B, 44(5):489-504, 1992.

[119] Marcia B Baker and Robert J Charlson. Bistability of CCN concentrations and thermodynamics in the cloud-topped boundary layer. $345: 142-145$, 1990.

[120] J Kazil, H Wang, G Feingold, A D Clarke, Jefferson Robert Snider, and AR Bandy. Modeling chemical and aerosol processes in the transition from closed to open cells during VOCALS-REx. Atmospheric Chemistry and Physics, 11(15):7491-7514, 2011.

[121] Karl D Froyd, Daniel M Murphy, Charles A Brock, Pedro CampuzanoJost, Jack E Dibb, Jose-Luis Jimenez, Agnieszka Kupc, Ann M Middlebrook, Gregory P Schill, Kenneth L Thornhill, et al. A new method to quantify mineral dust and other aerosol species from aircraft platforms using single-particle mass spectrometry. Atmospheric Measurement Techniques, 12(11):6209-6239, 2019.

[122] Sean Twomey. The nuclei of natural cloud formation part ii: The supersaturation in natural clouds and the variation of cloud droplet concentration. Geofisica pura e applicata, 43(1):243-249, 1959. 